\title{
Difusión Selectiva de Hidrocarburos en Zeolitas de Poro Pequeño a Partir de Técnicas de Simulación Molecular
}

Ciencias Químicas

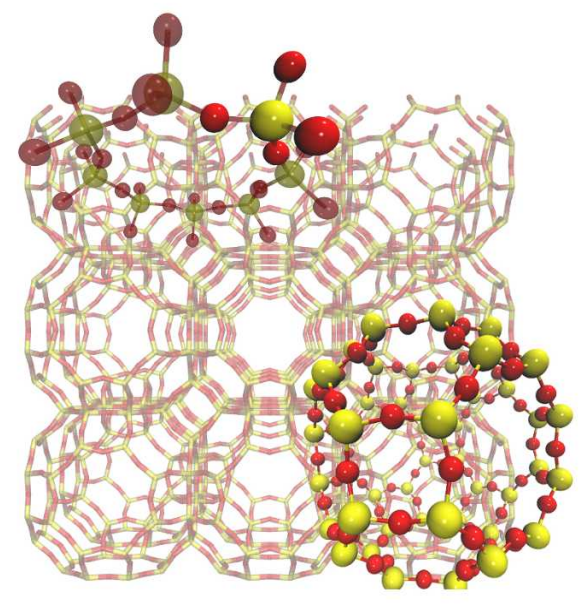

Tesis Doctoral

Aldo F. Combariza Montañez

Director

Dr. Germán I. Sastre Navarro

Valencia, 2011

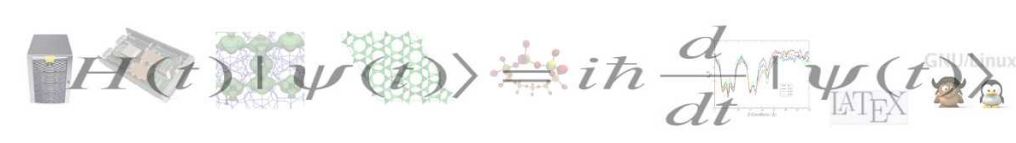



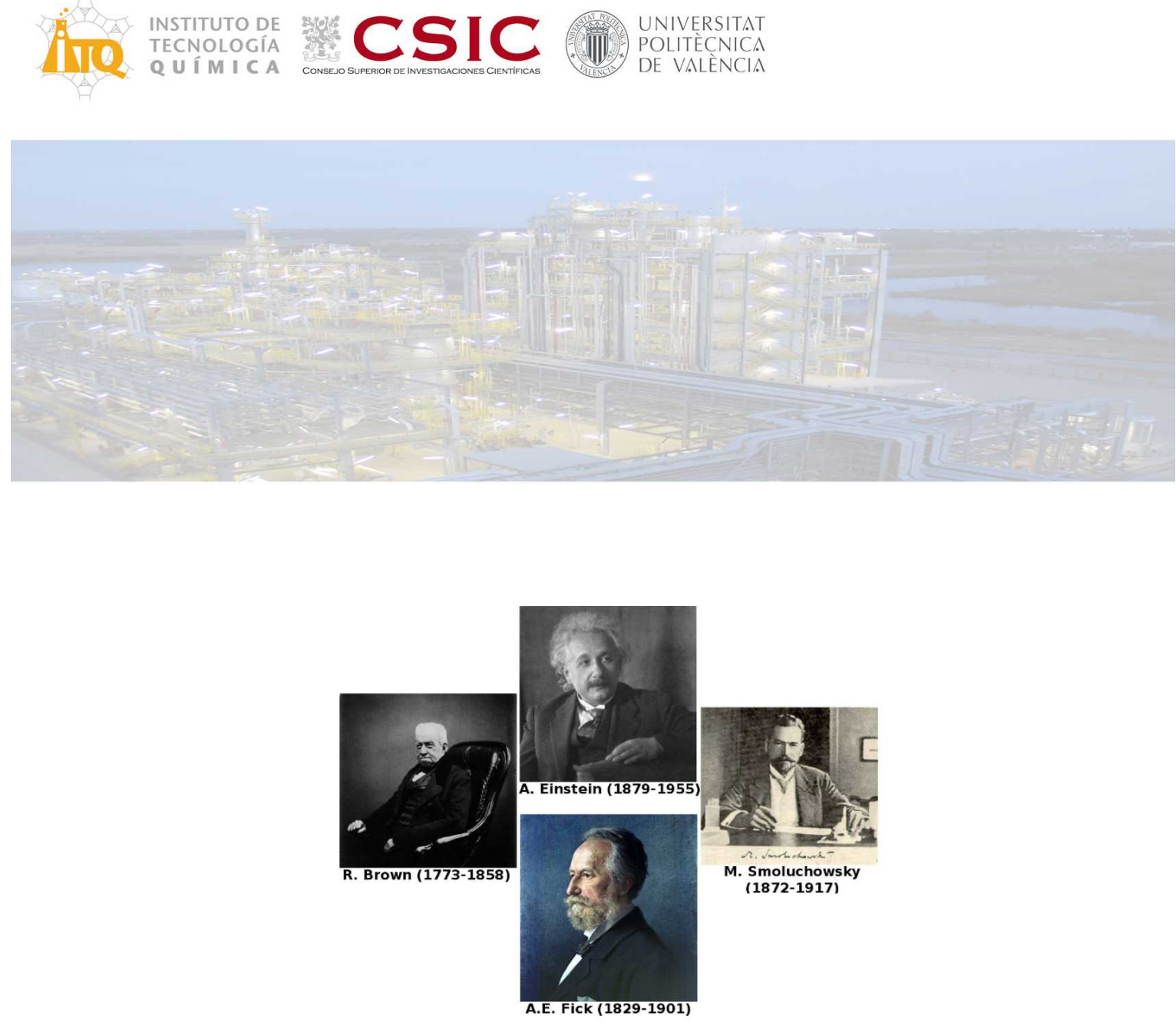

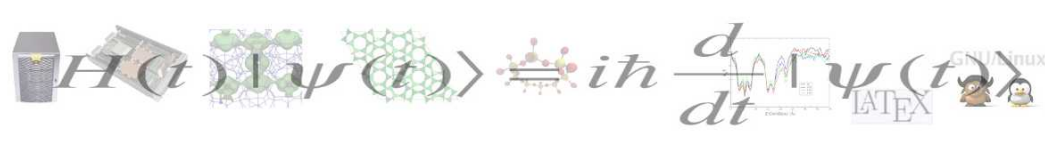


Difusión Selectiva de Hidrocarburos en Zeolitas de Poro Pequeño a Partir de Técnicas de Simulación Molecular

Aldo Fabrizzio Combariza Montañez

Instituto de Tecnología Química (UPV-CSIC) Universidad Politécnica de Valencia

Octubre de 2011 



\title{
Difusión Selectiva de Hidrocarburos en Zeolitas de Poro Pequeño a Partir de Técnicas de Simulación Molecular
}

\author{
Memoria Presentada por
}

\author{
Aldo Fabrizzio Combariza Montañez \\ Ingeniero Químico \\ Universidad Industrial de Santander (Colombia) \\ M. Sc. Química \\ University of Massachusetts, Amherst (USA)
}

Dirigida por

Dr. Germán Ignacio Sastre Navarro

Para Optar al Título de

Doctor en Ciencias Químicas

\section{Instituto de Tecnología Química (UPV-CSIC) \\ Universidad Politécnica de Valencia}




\section{Agradecimientos}

A Germán Sastre, director de mi tesis, por su incondicional apoyo y por su excepcional trabajo como tutor de mi trabajo doctoral.

A Avelino Corma, por darme la oportunidad de trabajar en el ITQ, y por ser una fuente inagotable de inspiración.

A Amparo Mifsud, por su ayuda y disposición, y por hacer del ITQ un lugar mucho mas amable.

A Jaime Primo, un agradecimiento especial por su paciencia y por sus consejos.

A Mónica, Inma y Marcos: su trabajo es altamente apreciado, su colaboración, sin duda alguna, hace mas fácil el camino.

A José Sanchez Marín, profesor de la Universidad de Valencia, por su diligencia y colaboración.

Un agradecimiento especial a Diego Gómez, por las muchas y fructíferas conversaciones sobre ciencia y computadores, pero sobre todo por ser un excelente compañero.

A Siris, Peter, Angeles, Mer, Tirso, Adela por hacer del laboratorio un lugar de trabajo muy agradable.

A Peter Vennestrøm una mención especial por su colaboración por hacer que el primer contacto que un grupo muy especial de niños tuvo con la ciencia fuera tan divertido y seguramente inolvidable.

The most exciting phrase to hear in science, the one that heralds new discoveries, is not 'Eureka!' but 'That's funny ...' Isaac Asimov 
Un agradecimiento muy especial a Yuriy Bushuev, por las enriquecedoras conversaciones que tuvimos mientras estuvo en el ITQ, y las que sin ninguna duda se darán en el futuro.

Mis agradecimientos no estarían completos si no incluyeran a la persona que me abrió las puertas de la química teórica y computacional, a Scott M. Auerbach, por mostrarme el camino que seguramente seguiré recorriendo en el futuro.

...Step by step, it must be done. And AC said,

LET THERE BE LIGHT!

And there was light...

Isaac Asimov (The Last Question)

A mis padres, por su constante e incondicional apoyo. 
Dedicado con mucho cariño a Aldo Jr. 


\section{Resumenes}




\section{Resumen}

En la industria petroquímica, olefinas como el etileno y propileno son materias primas muy importantes en la producción de polímeros tales como polietileno, polipropileno, estireno, etil benceno, dicloruro de etileno e isopropanol. La obtención de dichas olefinas se lleva a cabo por procesos de destilación que consumen enormes cantidades de energía, así como el diseño y utilización de equipos muy costosos. Una situación similar se da en procesos de rectificación y secado de gas natural, donde la separación de especies como $\mathrm{CO} 2$ y H20 consume una gran cantidad de recursos energéticos. Alternativas a los métodos de separación convencionales involucran el uso de tamices moleculares, membranas porosas o la utilización de líquidos iónicos para extracción reactiva. La tecnología posiblemente más prometedora envuelve el uso de tamices moleculares, debido al alto grado de desarrollo en las técnicas de síntesis, especialmente en el campo de las zeolitas, que han permitido una ingeniería cada vez mas detallada de materiales con características químicas y topológicas bien definidas.

El punto central del presente trabajo involucra la utilización de técnicas computacionales, especialmente en técnicas basadas en campos de fuerza, para lograr un entendimiento mas profundo de los fenómenos de transferencia de masa y energía que se dan en sistemas de tipo adsorbente-adsorbato. Zeolitas con poros conectados por ventanas compuestas de 8 unidades tetraédricas de $\mathrm{SiO} 4$, han sido reconocidas por su potencial para llevara cabo procesos de separación cinética de manera eficiente. Un parámetro muy importante que refleja el nivel de influencia del confinamiento ejercido por el material nanoporoso en la dinámica de especies adsorbidas es la "difusividad". La difusividad de las especies adsorbidas puede ser utilizada como parámetro de referencia para llevar a cabo la selección de materiales con potencial para llevar a cabo separaciones cinéticas. Este tipo de separaciones está basado en la diferenciación de la movilidad intracristalina en mezclas de especies con características químicas similares, únicamente en base al tamaño molecular. 


\section{Resum}

En la indústria petroquímica, olefines com l'etilè i propilè són matèries primeres molt importants en la producció de polímers com per eixemple polietilè, polipropilè, estirè, etil benzè, diclorur d'etilè i isopropanol. L'obtenció d'aquestes olefines es porta a terme per processos de destil-lació que consumeixen enormes quantitats d'energia, així com el disseny i utilització d'equips molt costosos. Una situació similar es dóna en processos de rectificació i assecat de gas natural, on la separació d'espècies com $\mathrm{CO} 2$ i H20 consumeix una gran quantitat de recursos energètics. Alternatives als mètodes de separació convencionals involucren l'ús de tamisos moleculars, membranes poroses o la utilització de líquids iònics per a extracció reactiva. La tecnologia possiblement més prometedora envolta l'ús de tamisos moleculars, a causa de 1?alt grau de desenvolupament en les tècniques de síntesi, especialment en el camp de les zeolites, que han permès una enginyeria cada vegada més detallada de materials amb característiques químiques i topològiques ben definides.

El punt central d'aquest treball involucra la utilització de tècniques computacionals, especialment en tècniques basades en camps de força, per aconseguir una entesa més profund dels fenòmens de transferència de massa i energia que es donen en sistemes de tipus absorvent-adsorbato. Zeolites amb porus connectats per finestres compostes de 8 unitats tetraèdriques de $\mathrm{SiO} 4$, han estat reconegudes pel seu potencial per dur a terme processos de separació cinètica de manera eficient. Un paràmetre molt important que reflecteix el nivell de influència del confinament exercit pel material nanoporos en la dinàmica d'espècies adsorbides és la "difusivitat". La difusivitat de les espècies adsorbides pot ser utilitzada com a paràmetre de referència per dur a terme la selecció de materials amb potencial per separacions cinètiques. Aquest tipus de separacions està basat en la diferenciació de la mobilitat intracristalina en mescles d'espècies amb característiques químiques similars, únicament sobre la base a la mida molecular. 


\section{Summary}

The production of raw materials such as ethylene and propylene is one of the main activities of the petrochemical industry. These materials are essential for the production of polyethylene, polypropylene, styrene and other important polymers. The precursor oleffins are obtained through energetically costly separation processes, e. g. cryogenic distillation, and require the design and use of expensive equipment. A similar situation occurs in the process of rectification and natural gas drying, where the separation of $\mathrm{CO} 2$ and $\mathrm{H} 2 \mathrm{O}$ requires, huge amounts of energy. Alternative separation methods involve the use of molecular sieves, porous membranes or ionic liquids, the latter used in reactive distillation processes. However, the most promising separation technology involves the use of molecular sieves, mainly due to the high degree of development in synthesis techniques, which allows materials with well defined chemical and topological features to be synthesized

The present work involves the use of computational techniques, specifically techniques based on force-fields, to shed light on the basic mass and energy transport phenomenology in zeolite-hydrocarbon systems. Specifically we are interested in zeolites with pore and channel dimensions close to the size small hidrocarbons (C1-C3). Zeolites with windows composed by eigth tetrahedral $\mathrm{SiO} 4$ units have been recognized as potential candidates to carry out kinetic separation processes. The attention is drawn towards the diffusivity, parameter that reflects the degree of confinement imposed by the topology of the adsorbant on the adsorbed species. Thus, the diffusivity can be used as a reference parameter to select materials able to achieve the goals of specific kinetic based separations. 


\section{Índice general}

Agradecimientos

Dedicatoria

Resumenes $\mathbf{v}$

Resumen ..................... VII

Resum . . . . . . . . . . . . . . . . . IX

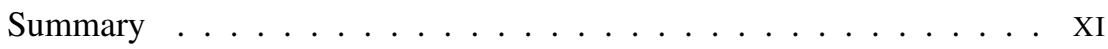

1. Introducción 1

1.1. Zeolitas y su Papel en Procesos de Separación . . . . . . . . . . . 3

1.2. Difusión: una Visión Fundamental . . . . . . . . . . . . . 5

1.3. ¿Cual es el Papel de las Técnicas Computacionales? . . . . . . . . 8

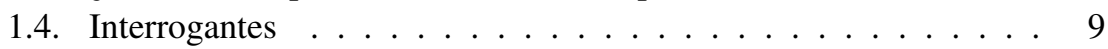

1.5. Estructura de la Tesis . . . . . . . . . . . . . . . . . 11

2. Difusión: Fundamentos y Métodos Computacionales 13

2.1. Difusión: Fundamentos Teóricos . . . . . . . . . . . . . . 15

2.1.1. Dependencia con la Concentración . . . . . . . . . . 16

2.1.2. Dependencia con la Temperatura . . . . . . . . . . . 18

2.1.3. Einstein y Movimiento Browniano . . . . . . . . . . . 18

2.2. Mecánica Estadística . . . . . . . . . . . . . . . . . . 19

2.3. Dinámica Molecular . . . . . . . . . . . . . . . . 21

2.3.1. Ecuaciones de movimiento de Newton . . . . . . . . . . 22

2.3.2. Condiciones Periódicas de Frontera . . . . . . . . . . . 24

3. Campos de Fuerza para el Estudio de Polimorfos Silíceos 27

3.1. Un Vistazo a la Literatura . . . . . . . . . . . . . . . . . . . . 29

3.2. Seleccion de los Campos de fuerza . . . . . . . . . . . . . 30

3.3. Modelos de Polimorfos Silíceos . . . . . . . . . . . . . . . 34 
3.4. Propiedades Calculadas . . . . . . . . . . . . . . . 35

3.4.1. Propiedades Estructurales . . . . . . . . . . . . 35

3.5. Campos de Fuerza . . . . . . . . . . . . . . . . . 38

3.5.1. Términos Electrostáticos . . . . . . . . . . . . . 38

3.5.2. Términos Enlazantes . . . . . . . . . . . . . . . . 42

3.5.3. Términos No-Enlazantes . . . . . . . . . . . . . . . 43

4. Difusión en Zeolitas: Influencia de la Flexibilidad de la Red 45

4.1. Modelos . . . . . . . . . . . . . . . . . . . . 47

4.2. Métodos . . . . . . . . . . . . . . . . . . . 48

4.2.1. Detalles de Cálculo . . . . . . . . . . . . . . . . . 48

4.2.2. Potencial Interatómico . . . . . . . . . . . . . . . . 49

4.3. Resultados y Discusión . . . . . . . . . . . . . . . . . 51

4.3.1. Variación Estructural con la Temperatura . . . . . . . 51

4.3.2. Dinámica de las Ventanas . . . . . . . . . . . . . 54

4.3.3. Difusión . . . . . . . . . . . . . . 57

4.4. Conclusiones . . . . . . . . . . . . . . 61

5. Difusión de Hidrocarburos en Zeolitas 63

5.1. Estructuras Seleccionadas . . . . . . . . . . . . . . . . . 64

5.2. Metodología . . . . . . . . . . . . . . . . 66

5.2.1. Detalles de Cálculo . . . . . . . . . . . . . . . 66

5.2.2. Superficie de Energía Potencial . . . . . . . . . . . 67

5.3. Difusión en Zeolitas . . . . . . . . . . . . . . . . . . . . . 68

5.3.1. Dinámica en la Zeolita Si-LTA . . . . . . . . . . . 68

5.3.2. Dinámica en la Zeolita Si-IHW . . . . . . . . . . . 73

5.3.3. Dinámica en la Zeolita Si-ITE . . . . . . . . . . . . . 77

5.3.4. Comparación de las características difusivas en Si-LTA,

Si-IHW y Si-ITE . . . . . . . . . . . . . . . . . . 79

5.4. Conclusiones . . . . . . . . . . . . . 80

6. Rol de la Superficie en el Proceso de Adsorción de Metano en la Zeo$\begin{array}{lr}\text { lita Si-LTA } & 83\end{array}$

6.1. Modelo del Sistema . . . . . . . . . . . . . . . . . . . 85

6.2. Detalles de la Metodología de Cálculo . . . . . . . . . . . . . 86

6.3. Resistencia Superficial al Transporte de Masa . . . . . . . . . 88

6.4. Resultados y Discusión . . . . . . . . . . . . . . . . . . 89

6.4.1. Zonas de Probabilidad de Ocupación y Energía Libre . . . 90

6.4.2. Permeabilidad Superficial _. . . . . . . . . . 93

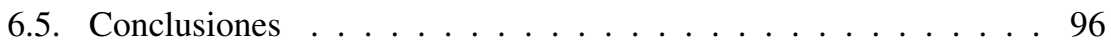


A. Parámetros Estructurales, Mecánicos y Dieléctricos de Estructuras Seleccionadas $\quad 103$

A.1. Cuarzo- $\alpha$ : Parámetros Calculados y Experimentales . . . . . . . . 104

A.2. Cuarzo- $\beta$ : Parámetros Calculados y Experimentales . . . . . . . 106

A.3. Coesita: Parámetros Calculados y Experimentales . . . . . . . 108

A.4. Si-LTA: Parámetros Calculados y Experimentales . . . . . . . 110

A.5. Zeolita SSZ-73: Parámetros Calculados y Experimentales . . . . . 112

A.6. Si-CHA: Parámetros Calculados y Experimentales . . . . . . . 114

A.7. ITQ-1: Parámetros Calculados y Experimentales . . . . . . . . . 116

B. Lista de Artículos Publicados 119

B.1. Artículos que Hacen Parte de la Tesis . . . . . . . . . . . . . . 119

B.2. Artículos que no Hacen Parte de la Tesis . . . . . . . . . . . . . 119

B.3. Artículos Publicados Durante M.Sc. . . . . . . . . . . . . 120 



\section{Capítulo 1}

\section{Introducción}

En este capítulo se plantean, de forma resumida, la motivación y los principios teóricos y prácticos utilizados para el desarrollo de la presente tesis. Inicialmente se hace una introducción a los materiales microporosos, principalmente Zeolitas, que son objeto del presente estudio. Seguidamente, se pone de manifiesto la importancia de los fenómenos de de difusión y adsorción en el campo de lo procesos de separación, se describen de forma general los modelos y los métodos de simulación aplicados y se plantean los interrogantes a los que se busca respuesta. Finalmente, se describe la estructura de la tesis.

En la industria petroquímica, olefinas como el etileno y propileno son materias primas muy importantes en la producción de polímeros tales como polietileno, polipropileno, estireno, etil benceno, dicloruro de etileno e isopropanol. Normalmente se utilizan procesos de destilación criogénica para la obtención de olefinas. Sin embargo, este tipo de procesos tiene la desventaja de requerir enormes cantidades de energía, alcanzando valores cercanos al $30 \%$ del total de energía consumida en planta (Figura 1.1). ${ }^{1-4}$ Recientes estimaciones calculan un ahorro potencial de energía del $15 \%$ como consecuencia de mejoras en los procesos de compresión y de separación. Una situación similar se da en procesos de rectificación y secado de gas natural, donde la separación de especies como $\mathrm{CO}_{2}$ y $\mathrm{H}_{2} \mathrm{O}$ consumen una gran cantidad de recursos energéticos. ${ }^{4}$

Rutas alternativas a los métodos de separación convencionales involucran el uso de tamices moleculares, membranas porosas o la utilización de líquidos iónicos para extracción reactiva. ${ }^{5-9}$ Una de las tecnologías más prometedoras es la de tamizado molecular, debido al alto grado de desarrollo en las técnicas de síntesis de materiales, especialmente en el campo de las zeolitas, que han permitido una ingeniería cada vez más detallada de materiales con carácterísticas químicas y 


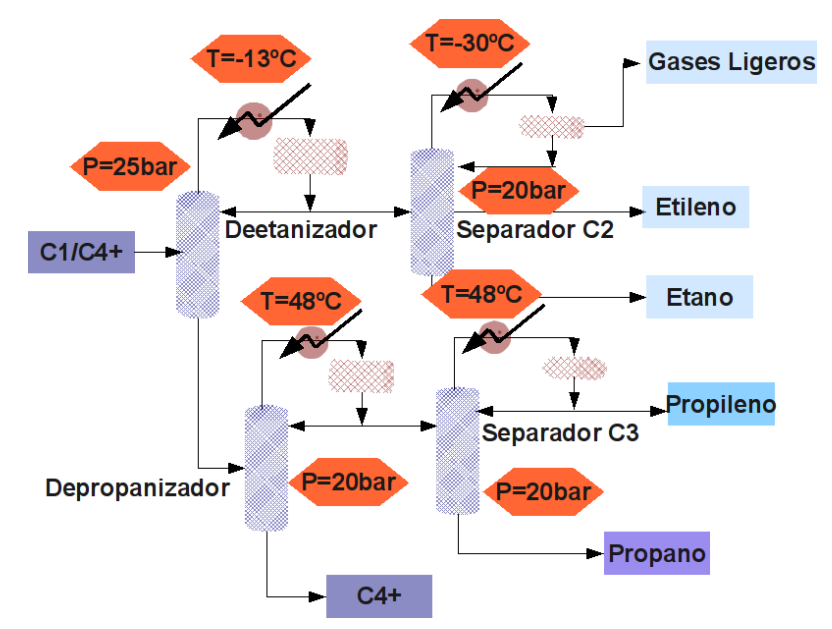

Figura 1.1: Esquema simplificado del proceso de destilación criogénica convencional para la separación de mezclas olefina/parafina. Se destacan las condiciones de temperatura (baja, para la separación de fracciones ligeras) y presión (elevada, en todos los casos) típicas del proceso. ${ }^{14}$

topológicas bien definidas. ${ }^{10-13}$

El uso de principios científicos rigurosos en áreas aplicadas de la tecnología química, particularmente en procesos que involucran transferencia de masa, ha sido desde sus inicios una tarea desafiante. Los mayores avances en este campo han surgido a partir de la utilización de modelos fenomenológicos, los cuales han relegado a un segundo plano la utilización de modelos derivados a partir de un conocimiento fundamental de los principios físicos, con una visión más rigurosa, controlantes de los fenómenos básicos de transferencia de materia o de la actividad catalítica.

La explicación esta en que la investigación experimental avanzada en el campo es sin duda alguna costosa en términos económicos y de tiempo. Sin embargo, en los últimos años, el desarrollo de nuevas técnicas analíticas experimentales y computacionales ha permitido profundizar en la fenomenología de los procesos de transferencia de masa a escala microscópica. Este conocimiento es fundamental, ya que en materiales porosos el efecto de confinamiento impuesto por la estructura afecta de manera considerable, y muchas veces sorprendente, las propiedades termodinámicas y de transporte de los adsorbatos.

En este capítulo se da un vistazo general a los fenómenos de transferencia de masa en materiales zeolíticos, principalmente aquellos relacionados con procesos 
de difusión, así como a las técnicas computacionales utilizados para su estudio, una descripción mucho más detallada es dada en capítulos posteriores. Seguidamente, se plantean los interrogantes a los que se busca respuesta y se da un bosquejo de la estructura de la tesis.

\subsection{Zeolitas y su Papel en Procesos de Separación}

Las zeolitas son materiales microporosos constituidos por unidades tetrahédricas de óxido de silicio o aluminio, interconectadas de forma tal que permiten la creación de complejas estructuras con canales y cavidades de dimensiones moleculares. Es precisamente el tamaño de estas cavidades y canales, además de su dimensionalidad, lo que los convierte en excelentes candidatos para aplicaciones en la industria química y para su utilización en aplicaciones relacionadas con intensificación de procesos, química verde, materiales híbridos, medicina, alimentación animal, aplicaciones opto-electrónicas, microsistemas de reacción y detección, y nanotecnología. ${ }^{15-17}$

La importancia de estos materiales ha hecho que el concepto de zeolita haya sido extendido para definir genéricamente los llamados materiales porosos de diseño (ver Figura 1.2), dentro de los cuales se destacan: tamices moleculares, sólidos porosos de coordinación, tales como los enrejados metalo-orgánicos (MOFs, por sus siglas en inglés) o más adecuadamente MOFs isoreticulares (IRMOFs), MOFs microporosos (microporosos) o polímeros coordinados porosos (PCP), carbones porosos, óxidos derivados de sol-gel y sales porosas heteropolianiónicas. ${ }^{18}$ Consecuentemente, el término 'material zeolítico' es frecuentemente utilizado en la literatura para definir un amplio espectro de materiales, con unas características específicas basadas en las propiedades estructurales de las zeolitas.

En el momento de escribir esta tesis, aparecen registradas en la página oficial de la Asociación Internacional de Zeolitas (IZA, por sus siglas en inglés) 194 tipos de estructuras zeoliticas (Ver Fig. 1.3) con códigos asignados por la IUPAC. ${ }^{19}$ Dentro de esta gran variedad de formas, un subgrupo compuesto por zeolitas con poros conectadas a través de ventanas de tamaño pequeño destaca por sus posibilidades de aplicación en el campo de los procesos de separación. Zeolitas con poros separados por anillos formados de 8 unidades tetraédricas de $\mathrm{SiO}_{4 / 2}$ (8 Member Ring, 8-MR) han sido recientemente reconocidas como candidatos potenciales para separar mezclas de compuestos con diámetros cinéticos que están en el mismo rango de las dimensiones de las ventanas. ${ }^{13,20-22}$

Ejemplos de este tipo especial de zeolitas son las recientemente sintetizadas ITQ-3 (ITE), ${ }^{21}$ ITQ-12 (ITW) ${ }^{22}$ e ITQ-32 (IHW), ${ }^{11}$ las cuales han mostrado ser potencialmente útiles en la separación de mezclas de hidrocarburos que presentan propiedades químicas y tamaños similares. Aún más sobresaliente, es su capaci- 

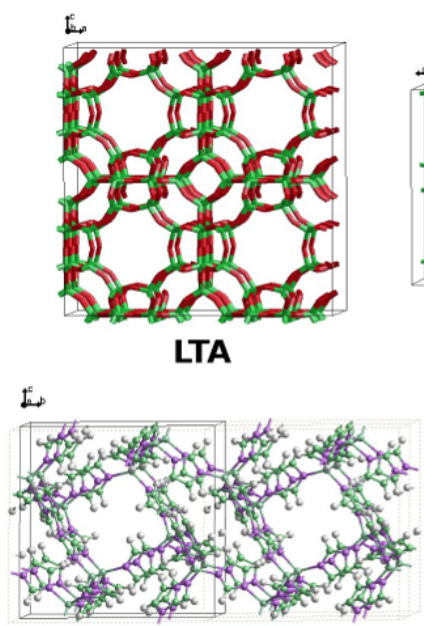

ZIF-1

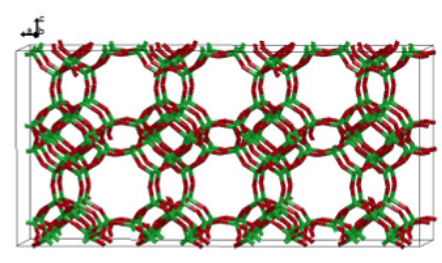

ITE

L

ZIF-8

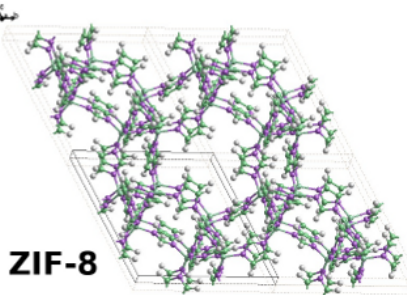

Figura 1.2: Estructura cristalina de dos tipos diferentes de materiales nanoporosos zeotípicos: (arriba) zeolitas con estructuras de tipo ITE y LTA, y redes zeolíticas de imidazolato ZIF-1 y ZIF-8 (abajo).

dad de diferenciar entre mezclas de olefinas y parafinas, utilizandose para este fin estructuras formadas enteramente por silice. ${ }^{23}$ Este tipo de separaciones no es posible con zeolitas catiónicas, debido a la oligomerización de las olefinas, que a su vez causa el bloqueo de las cavidades internas del material y el consecuente deterioro en la capacidad de separación. Los procesos de separación basados en fenómenos de adsorción se pueden clasificar en tres categorías principales: estéricos, cinéticos y de equilibrio. El efecto estérico se da a partir de las propiedades de selección molecular de la zeolita, en cuyo caso solo moleculas con el tamaño y forma adecuada pueden acceder al interior del adsorbente, excluyendo así totalmente las demas especies. Las separaciones cinéticas se llevan a cabo gracias a diferencias representativas en los coeficientes de difusión de los distintos adsorbatos. Por último, las separaciones basadas en adsorción a condiciones de equilibrio de la mezcla son conocidas como separaciones en equilibrio, y representan la gran mayoría de los procesos de separación adsortivos.

Nuestro interés inmediato se centra en el grupo de separaciones cinéticas, principalmente en separaciones de adsorbatos en el rango de tamaños correspondiente a zeolitas con ventanas de 8-MR. La figura 1.4 muestra, de forma comparativa, el tamaño crítico de los anillos de materiales zeolíticos respecto a los tamaños cinéticos de un grupo de compuestos relevantes en la industria (petro- 


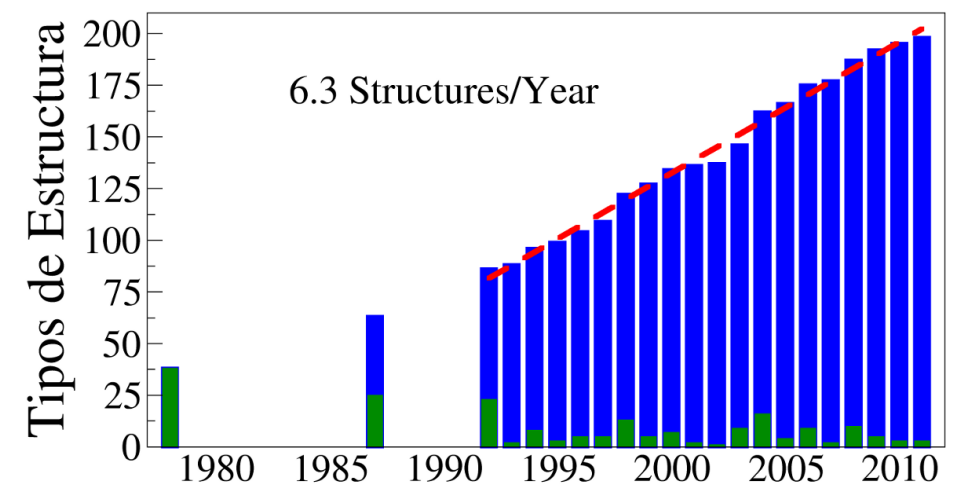

Figura 1.3: Evolución en el numero de tipos de redes zeolíticas de acuerdo al año de aprobación de la estructura por la Comisión de Estructuras de la IZA, representado por las barras de color negro. ${ }^{19}$ Las barras verdes muestran la sumatoria de tipos de estructuras a través del tiempo hasta el presente. La linea discontinua representa un ajuste lineal de los datos acumulados, cuya pendiente de 6.3 correspondería al número promedio de tipos de estructuras aprobados por la IZA anualmente desde 1992.

)química, esto con el fin de dar una idea aproximada de los tamaños y tipos de moleculas a los que nos referiremos en capítulos posteriores.

\subsection{Difusión: una Visión Fundamental}

El estudio de la transferencia de masa, y específicamente de la difusión, tiene sus inicios en las últimas décadas del siglo XIX (Ver Fig. 5). El trabajo pionero de Adolf Fick, ${ }^{24,25}$ basado en los experimentos de Thomas Graham ${ }^{26,27}$ sobre difusión en gases y líquidos, publicado en 1855, sienta las bases matemáticas para la descripción fenomenológica de la difusión. Posteriormente Dushman y Langmuir, ${ }^{28,29}$ en 1922, reconocen la dependencia de la difusividad respecto a la temperatura, y toman prestado el concepto desarrollado tres décadas antes por Svante Arrhenius ${ }^{30}$ para describir el carácter activado de los procesos difusivos.

Una visión teoricamente más rigurosa de la difusión fue desarrollada por Albert Einstein, ${ }^{31-34}$ con base en los experimentos descritos por Robert Brown ${ }^{35-37}$ sobre el movimiento irregular y continuo de pequeñas partículas suspendidas en un líquido, más conocido como movimiento Browniano (Ver Figura 1.6. La idea de que la posición de las moléculas y no la velocidad de las mismas es la propiedad básica que describe la dinámica de la difusión es la idea más destacada de su trabajo en este campo. 


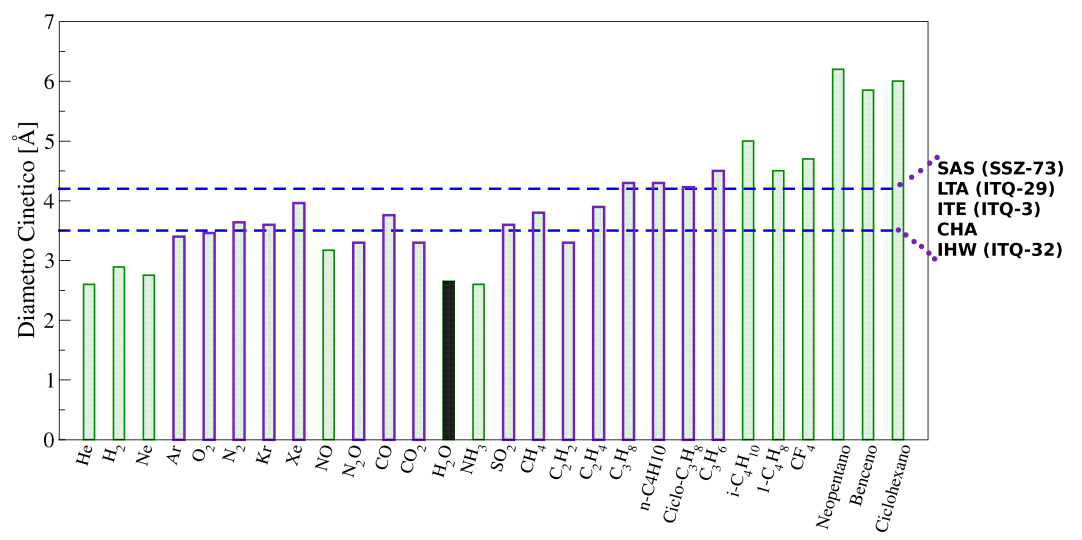

Figura 1.4: Diametros cinéticos de hidrocarburos en comparación con el tamaño crítico de la ventana de 8-ring para diferentes zeolitas. Las zeolitas están listadas en función decreciente del diámetro crítico. Zeolita SSZ-73 $r_{c}=4,2$, ITQ-29 $r_{c}=4,0$, ITQ-3 $r_{c}=3,8$, CHA $r_{c}=3,7$, ITQ-32 $r_{c}=3,5$.

Independientemente, el científico polaco Marian von Smoluchowsky, ${ }^{38,39} \mathrm{pu}-$ blicó conclusiones similares en 1906, partiendo de postulados tomados de la teoría cinética, a diferencia de los postulados termodinámicos de Einstein. La verificación experimental de la teoría llegaría poco después, cuando en 1908 el físico francés Jean Baptiste Perrin publica una serie resultados experimentales confirmando las predicciones hechas por la relación de Einstein-Smoluchowsky. ${ }^{40-42}$ Este sólido cuerpo teórico y experimental ha servido como base para estudios modernos de fenómenos difusivos, y a la vez han servido para el desarrollo de nuevas técnicas experimentales.

A diferencia de la difusión en gases y líquidos, para los que es relativamente sencillo el cálculo de parámetros de difusión, la difusión en materiales zeolíticos representa un caso muy especial, debido a la gran influencia ejercida por la estructura sobre la movilidad del adsorbato. La interacción constante entre el adsorbato y las paredes del sólido poroso se suman a la influencia ejercida por la concentración y la temperatura, lo cual no ocurre en sistemas puramente líquidos o gaseosos.

Los efectos de la superficie en la difusividad del adsorbato estarán controlados por las características topológicas del material, como el tamaño del poro o la dimensionalidad de la red de canales internos del material. Esta dependencia de la estructura sobre la difusión genera tipos especiales de difusión que son exclusivos de los materiales porosos. Un ejemplo es la difusión "en fila" o "single file 


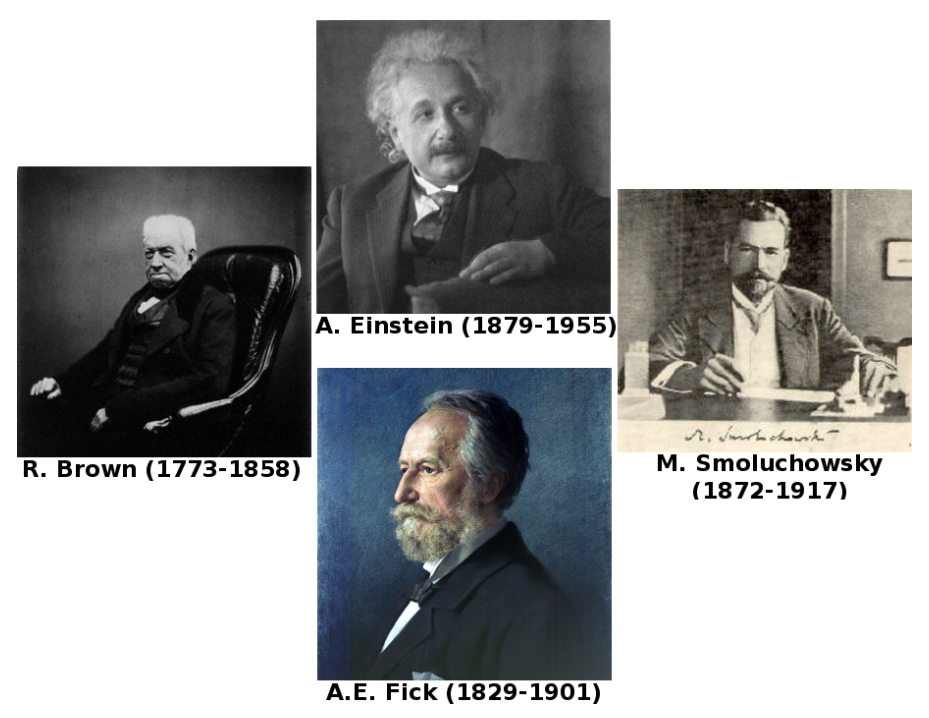

Figura 1.5: Pioneros en el estudio de los fenómenos de transferencia de masa.

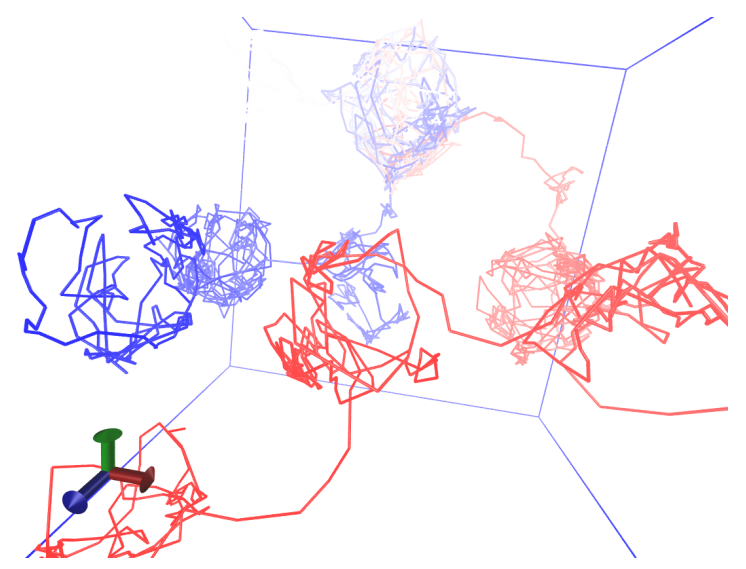

Figura 1.6: Representación del movimiento de tipo Browniano experimentado por una molécula de Metano (centro de masa) adsorbida en la zeolita ITQ-29 a una temperatura de $150 \mathrm{~K}$. La escala de colores corresponde a la evolución en el tiempo, aumentando en el sentido azul-rojo. La intensidad de los colores refleja la posición respecto al plano frontal. 
diffusion", que se presenta en sistemas unidimensionales donde las moléculas adsorbidas no pueden sobrepasarse unas a otras, quedando reducida su dinámica a un movimiento ordenado. Otro ejemplo se debe a los efectos de levitación, que se presentan en sistemas donde las paredes de la red rodean a las moléculas de forma tal que las interacciones se balancean y la molécula experimenta un movimiento acelerado, que se traduce en coeficientes de difusión muy altos.

La transferencia de masa en nanoporos se cuantifica en términos de la difusividad. Este parámetro puede ser medido a partir de técnicas experimentales, que dependiendo de su nivel de complejidad pueden llegar a medir fenómenos de difusión a nivel intracristalino. Sin embargo, no existe un modelo teórico general que permita su predicción, dada la dificultad de relacionarla con mecanismos microscópicos subyacentes. Desde una visión aplicada, la importancia de determinar y describir los procesos de transferencia de masa en materiales porosos es producto de la necesidad de entender a fondo su papel en procesos industriales de separación y catálisis. Este entendimiento es básico para la optimización y el desarrollo de tecnologías basadas en estos materiales.

\section{3. ¿Cual es el Papel de las Técnicas Computacionales?}

El impresionante desarrollo en la capacidad computacional de las últimas decadas ha abierto las puertas a una nueva rama de las ciencias, a la que se refiere a menudo como "la tercera forma" de hacer ciencia, ${ }^{43,44}$ y que se basa en la experimentación computacional, o experimentación "In Silico". ${ }^{45}$ La forma de llevar a cabo un experimento computacional está dada por el modelado de un sistema real, a partir de principios teóricos sólidos, y la posterior simulación de su comportamiento utilizando algoritmos matemáticos de cálculo. Por supuesto, tanto el modelo como el método de solución dependen del propósito de la simulación.

Los métodos computacionales desarrollados para la descripción del comportamiento de la materia a nivel molecular pueden dividirse en dos grandes tipos: métodos $A b$ Initio y métodos atomísticos. Las técnicas $a b$ initio describen la estructura electrónica y la reactividad de sistemas moleculares a partir de postulados mecano-cuánticos, ${ }^{46,47}$ mientras que los métodos atomísticos utilizan los principio de la mecánica clásica para simular sistemas a nivel atómico, ${ }^{48}$ pudiendo determinarse de esta manera propiedades macroscópicas a partir de postulados de la física estadística. ${ }^{49-51}$ los métodos ab initio solo requieren cono entrada inicial las coordenadas nucleares del sistema, mientras que los métodos clásicos requieren de un conjunto de condiciones iniciales y de la descripción detallada de las interacciones inter-atómicas.

La elección de la metodología computacional para el estudio de un problema determinado dependerá, tanto del tamaño del sistema a considerar como de 
la capacidad computacional de que se disponga. Así, los métodos ab initio están restringidos a sistemas de unas cuantas decenas de átomos, debido a las complejidad de los principios mecano-cuánticos, mientras que los métodos atomísticos manejan fácilmente sistemas con miles a millones de partículas. En este trabajo, se utilizan métodos atomísticos y principios mecano-estadísticos para estudiar el comportamiento de moléculas confinadas dentro de materiales zeolíticos, con énfasis en el estudio del comportamiento difusivo de los adsorbatos. ${ }^{52-54}$

De manera paralela a las técnicas experimentales, las técnicas computacionales basadas en métodos atomísticos han venido desarrolándose y mostrando su utilidad en el estudio de procesos de separación en materiales porosos. Prueba de ello es el actual grado de desarrollo y complejidad de algoritmos y métodos computacionales para el estudio de fenómenos de adsorción y difusión bajo condiciones de confinamiento. Técnicas como la Dinámica Molecular (DM) y el método de Monte Carlo (MC) han sido fundamentales para la correcta interpretación de resultados experimentales, muchas veces ambiguos o contradictorios. El objetivo final de estos métodos es la simulación de la evolución dinámica de un sistema compuesto por modelos de uno o más adsorbatos dentro de un solido cristalino con canales y poros de tamaño molecular.

La herramienta más adecuada para estudiar procesos de separación en condiciones de confinamiento es, sin duda alguna, la Dinámica Molecular (DM). Esta técnica se basa en propagar en el tiempo las ecuaciones clásicas de movimiento, partiendo de un modelo matemático representativo de las fuerzas interatómicas existentes utilizando un conjunto adecuado de condiciones de frontera. Por otro lado, los procesos de adsorción son principalmente estudiados a través de búsquedas estocásticas, basadas en muestreos del espacio configuracional, antes que del temporal. La técnica de MC, útil en el estudio de propiedades termodinámicas de adsorbatos, isotermas de adsorción y calores de adsorción, ha sido también exitosamente utilizada para obtener detalles acerca del acomodamiento a nivel molecular de adsorbatos en los poros de zeolitas, información necesaria para explicar el complejo comportamiento de moléculas en condiciones de confinamiento.

\subsection{Interrogantes}

La utilización de la técnica de DM impone el uso de modelos atomísticos de interacción intra e intermolecular. Normalmente las interacciones intermoleculares están dadas por a terminos no enlazantes de dispersion y repulsion de corto alcance, o interacciones de van der Waals. Este tipo de interacciones suelen ser modelados usando potenciales sencillos, de tipo Lennard-Jones, con parámetros de energía y tamaño calculados a partir de las conocidas reglas de mezcla de Lorenz-Berthelot. 
Sin embargo, el reto definitivo para la utilización exitosa de la DM está en la correcta implementación de modelos interatómicos, que describan correctamente la física de las interacciones intramoleculares. El caso de los hidrocarburos de cadena corta en los que estamos interesados en el presente trabajo no representa un problema mayor, ya que existen modelos con una larga trayectoria de uso exitoso en aplicaciones computacionales. Por el contrario, la existencia de una gran variedad de modelos para representar las interacciones intramoleculares en estructuras zeoliticas exige un proceso de selección cuidadosa.

- El punto inicial que se plantea en esta tesis, es el de generar una herramienta para la selección del modelo más adecuado que represente las interacciones intramoleculares en la zeolita, y que se adecúe más convenientemente al tipo de cálculo que se plantea llevar a cabo. Con este fin en mente, se presenta el análisis riguroso de una serie de potenciales atomísticos desarrollados para la modelización de estructuras zeolíticas, modelos que discrepan en cuanto a complejidad en la forma funcional, en la forma de parametrización de dichas formas funcionales y que se evalúan en cuanto a su transferabilidad y a su habilidad para predecir valores experimentales.

Una vez identificado el modelo, o modelos a utilizar en la representación de las interacciones inter e intramoleculares, se plantea un punto que a pesar de haber sido objeto de varios estudios en el pasado, ha persistido a lo largo del tiempo como un interrogante en el campo de la DM.

- La influencia que tiene la forma de representar la red en los cálculos de coeficientes de difusión, más específicamente la influencia de la flexibilidad de la estructura para la correcta descripción de los parámetros difusivos de un sistema adsorbato-adsorbente.

Dentro del campo de las separaciones de mezclas de compuestos, se conocen estudios preliminares que muestran la capacidad de ciertos materiales, específicamente zeolitas de poro pequeño (8-MR), para la separación de compuestos con tamaños similares a las ventanas que comunican los poros. Sin embargo, el carácter cinético de dichas separaciones no está completamente claro.

- Para dar una base atomística sólida a las mediciones experimentales, se plantean una serie de cálculos que buscan la cuantificación de los coeficientes de difusión de diferentes adsorbatos en un grupo de zeolitas con 8-MR. Además de la cuantificación de los coeficientes de difusión, también se plantea la verificación del carácter activado del proceso difusivo en este tipo de materiales, lo cual involucra la simulación de la dinámica en términos de la temperatura. 
Finalmente, se analiza un factor que ha sido motivo de un renovado interés, esto es, la influencia de la superficie en los procesos de transferencia de masa. Los fenómenos de superficie han sido planteados como una de las principales causas de discrepancia entre las diversas técnicas experimentales de medición de la difusividad.

- Nuestro interés se centra en modelar y analizar tanto el proceso transitorio de adsorción como en el proceso de transferencia de masa en condiciones de equilibrio.

\subsection{Estructura de la Tesis}

Una vez planteadas de manera general las bases teóricas sobre los procesos de transferencia de masa en materiales nanoporosos, reconocidas las técnicas computacionales que servirán de herramienta para el desarrollo de este trabajo y planteados los interrogantes a responder, se hace necesario dar una descripción detallada, más rigurosa, de cada uno de los anteriores puntos. Para este fin, se describe a continuación el contenido de cada uno de los capítulos siguientes.

- En el Capítulo 2 se plasman los planteamientos teóricos, físicos y atomísticos, que sirven como base fundamental en la descripción del fenómeno de la difusión. Seguidamente, se plantean los modelos teóricos desarrollados y utilizados en el campo específico de la difusión en materiales nanoporosos, y se revisan las técnicas experimentales más importantes para el cálculo de parámetros difusivos. Finalmente, se da una descripción detallada del método de DM, que se utiliza para el desarrollo de esta tesis, y de su aplicación en el estudio de la difusión en el campo de los materiales zeolíticos, así como de los algoritmos existentes y los desarrollados ex professo para el el análisis de la información obtenida a partir de las simulaciones de DM.

- En el Capítulo 3 se da una descripción detallada de los modelos atomísticos utilizados para la representación de las interacciónes intra e intermoleculares. Inicialmente se presentan los modelos seleccionados, se analizan los términos funcionales que representan las interacciones físicas, principalmente las interacciones no enlazantes de dispersión y repulsión de corto alcance, o interacciones de van der Waals, así como las interaciones de largo alcance, dominadas por términos electrostáticos o Coulómbicos. La discusión final se da alrededor de la habilidad de los diferentes modelos para representar correctamente propiedades mecánicas, estáticas y propiedades dinámicas de estructuras silíceas representativas, así como de su eficiencia en términos de carga computacional. 
- En el Capítulo 4, se estudia la influencia de la flexibilidad de la estructura zeolítica en el cálculo de coeficientes de difusión de adsorbatos de tamaño similar a los diámetros críticos de estructuras seleccionadas con ventanas de 8-MR. La discusión de los resultados se hace en base a las discrepancias encontradas al modelar las estructuras con diferentes potenciales y que se observan en la prediccion de diferencias estructurales obtenidas con cada modelo.

- El Capítulo 5 se discute la aplicación del método de DM para dar una explicación atomística a mediciones experimentales que muestran la capacidad de zeolitas con 8-ring en procesos de separación de compuestos con tamaños similares a las ventanas que comunican los poros. El objetivo central es el de clarificar el carácter cinético de dichas separaciones. Inicialmente se da una descripción de las carácterísticas topológicas de los materiales seleccionados, se describen los modelos atomísticos escogidos para la representación de las interacciones intra e interatómicas, y se discuten los resultados con base en las diferencias en los coeficientes de difusión calculados y de los parámetros de energías de activación obtenidos.

- El Capítulo 6 se centra en el estudio de la influencia de la superficie en los procesos de transferencia de masa. Inicialmente se revisan los modelos existentes que describen teóricamente los fenómenos de adsorción superficial, así como de las técnicas experimentales utilizadas en el estudio de superficies. Seguidamente, se plantean los detalles de la estructura a estudiar, así como del modelo atomístico seleccionado para representar las interacciones intramoleculares del sistema. Por último, se muestran los resultados de la aplicación del método de DM y se analizan los datos usando un análisis mecano-estadístico que permite discernir las carácterísticas especiales de la superficie en el proceso de adsorción.

- Finalmente, el Capítulo 7 resume los puntos más sobresalientes de los ítems anteriores y sumariza las conclusiones que se obtuvieron a lo largo de esta investigación. 


\title{
Capítulo 2
}

\section{Difusión: Fundamentos y Métodos Computacionales}

\begin{abstract}
La difusión de especies adsorbidas en sistemas porosos se desvía del comportamiento en estado gaseoso o líquido de dichos adsorbatos. Estas diferencias, las cuales se hacen más marcadas a medida que el sistema de difusión se hace más restrictivo, plantean un reto al tratar de entender su comportamiento a nivel macroscópico. Para entender el comportamiento dinámico de este tipo de sistemas, es necesario entender los principios físicos que rigen su comportamiento. En este capítulo se introducen los conceptos fundamentales en que se basa el estudio de los procesos de transferencia de masa en el interior de materiales porosos. Además se exponen, de forma general, los principios de la mecánica estadística, los cuales son necesarios para entender el método computacional de la Dinámica Molecular y su aplicación a la difusión en sistemas nanoporo-adsorbato.
\end{abstract}

En sistemas gaseosos o líquidos, la dinámica del sistema está dictada por las colisiones intermoleculares que se dan como consecuencia de la energía térmica de cada molécula. La teoría cinética toma el aparentemente azaroso comportamiento de las moléculas en un sistema, y a partir de propiedades físicas como masa, momento y energía permite llevar a cabo generalizaciones con base en promedios estadísticos, pudiendo de esta forma hacer predicciones con un alto grado de precisión. El movimiento de especies químicas en el interior de materiales porosos, contrariamente al caso de gases y líquidos, introduce un factor de interacción entre la estructura del material y las moléculas adsorbidas. Este factor de interacción hace que la isotropía existente en la fase pura, gas o líquido, se pierda 
y por tanto la predicción de propiedades a través de la teoría cinética deja de ser posible.

Tal como se describió en la introducción, el proceso de transferencia de masa que ocurre en el interior de partículas nanoporosas puede ser cuantificado en términos de la difusión. El nivel de influencia entre la fase adsorbida y el adsorbente puede apreciarse en la 2.1, donde se describen los diferentes regímenes de difusión molecular en función del tamaño de poro del material adsorbente. ${ }^{55} \mathrm{~Pa}$ ra sistemas con poros grandes, o macroporos, del orden de $0.1 \mu \mathrm{m}(>100 \mathrm{~nm})$, las interacciones estarán principalmente dadas por colisiones entre las moléculas adsorbidas, y por tanto el comportamiento será similar al de la difusión molecular en gases. Los coeficientes de difusión en este tipo de sistemas están alrededor de $\operatorname{los} 10^{-5} \frac{\mathrm{m}^{2}}{\mathrm{~s}}$, con energías de activación bajas, del orden de unos pocos $\frac{\mathrm{kJ}}{\mathrm{mol}}$.

En sistemas con poros más pequeños, en el rango $20-100 \mathrm{~nm}$, pero aún con tamaño relativamente mayor al tamaño del adsorbato, la dinámica de la fase adsorbida empieza a depender en mayor grado de las interacciones con las paredes del adsorbente, hasta el punto en que la distancia promedio para el choque de una molećula con las paredes del adsorbato se hace menor que la distancia recorrida en la fase gaseosa para que se de una colisión entre dos moléculas. A partir de este punto se considera que la difusión entra en el régimen de Knudsen, y la dependencia de la difusión con las dimensiones del poro empieza a tomar el control de la dinámica del sistema.

Finalmente, cuando las dimensiones del poro alcanzan dimensiones moleculares la interacción adsorbato-adsorbente dictará la dinámica del sistema, debido a la constante interacción entre las paredes del material poroso y la fase adsorbida. El nivel de interacción en materiales zeolíticos se encuentra en este régimen, llamado de difusión configuracional, y cuya característica primordial está dada por valores bajos de difusión y altas energías de activación.

El valor de las difusividades en el régimen configuracional abarca un rango de valores muy grande, entre $10^{-8}$ y $10^{-20} \frac{\mathrm{m}^{2}}{\mathrm{~s}}$. El orden de magnitud de la difusividad está influenciado por las interacciones con la red y por el tamaño y la forma del adsorbato. En algunos sistemas con características especiales, tales como sistemas con canales unidimensionales, estas interacciones pueden llevar a movilidades de tipo "single file", que se caracteriza por el movimiento ordenado de moléculas que no pueden sobrepasarse unas a otras, o a la aparición de efectos de levitación, que aparece debido al cancelamiento de la interacción de la superficie por efectos de simetria en la posición del adsorbato. La dependencia entre estos factores hace que la predicción y conceptualización de la difusión en estos sistemas sea una tarea cuando menos complicada, ya que la relación entre difusión-temperatura o difusión-concentración no sige una regla general y depende específicamente del tipo de sistema adsorbato/adsorbente. 


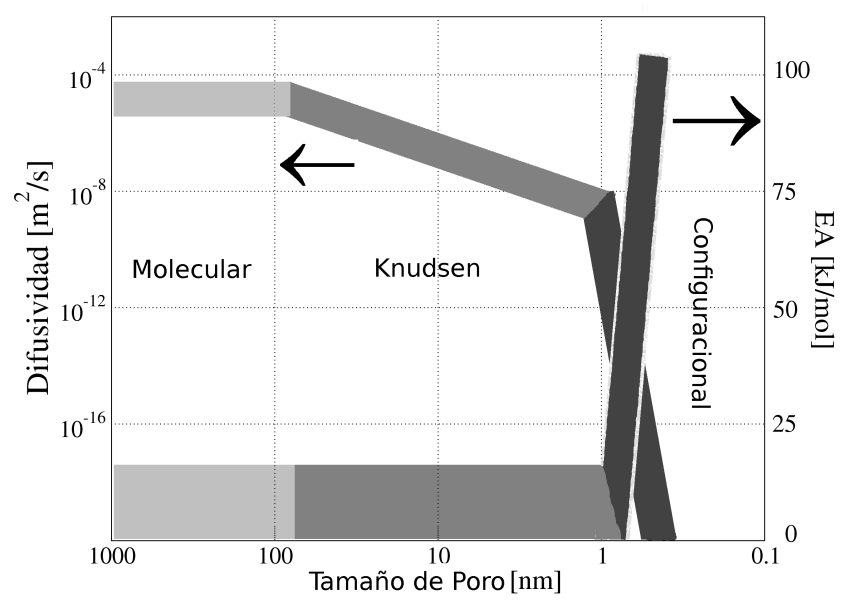

Figura 2.1: Regímenes de difusión en función del tamaño de poro del material. ${ }^{55}$

\subsection{Difusión: Fundamentos Teóricos}

Tal como se describió en la introducción, los principios teóricos de la difusión fueron planteados a mediados del siglo XIX por Adolf Fick. ${ }^{56}$ La descripción del flujo $(J)$ de una especie química, en función de la variación de la concentración de dicha especie a lo largo de una coordenada espacial $x$, está dada por la primera ley de Fick,

$$
J=-D_{t}\left(\frac{\partial C}{\partial x}\right)
$$

donde $D_{t}$ es el coeficiente de difusión para el transporte de masa en el sistema. Asumiendo que el coeficiente de difusión es independiente de la concentración, y asumiendo la isotropía de la difusión con respecto al material poroso, podemos definir la dependencia de la concentración con respecto al tiempo como

$$
\frac{\partial C}{\partial t}=-D_{t}\left(\frac{\partial^{2} C}{\partial x^{2}}\right)
$$

ecuación que se conoce como la segunda ley de Fick. A pesar de que la primera ley de Fick define de forma general el avance difusional, su dependencia con la concentración no es, rigurosamente hablando, la fuerza motriz para el proceso de transporte. Al tratarse de un sistema que no está en equilibrio termodinámico, y que por consiguiente tenderá a aproximarse al equilibrio, la fuerza motriz 
estará dada por el potencial químico $\mu$. La relación de Onsager, derivada a partir de postulados termodinámicos, define explicitamente esta dependencia con el potencial químico de acuerdo a la ecuación

$$
J=-L\left(\frac{\partial \mu}{\partial x}\right)
$$

siendo $L$ el coeficiente fenomenológico de Onsager. ${ }^{57}$

\subsubsection{Dependencia con la Concentración}

El potencial químico, $\mu$, puede relacionarse con la concentración a través de la ecuación de Darken,

$$
\mu=\mu_{0}+R \cdot T \cdot \ln (p)
$$

considerando que el potencial está relacionado con el equilibrio en fase gaseosa, donde $p$ representa la presión parcial del componente. A partir de esta ecuación, podemos relacionar el coeficiente de difusión de Fick de la siguiente forma

$$
D_{t}=R T L\left(\frac{\partial \ln p}{\partial \ln C}\right)=D_{0}\left(\frac{\partial \ln p}{\partial \ln C}\right)=D_{0} \cdot \Gamma
$$

de donde obtenemos la relación entre el coeficiente de difusión de Fick y el coeficiente de Onsager. $D_{0}$ es conocido como el coeficiente de difusión corregido o coeficiente de Maxwell-Stephan y $\Gamma$ es el factor de corrección termodinámico, que define el nivel de desviación lineal entre la concentración y la presión. ${ }^{58,59}$

El coeficiente de difusión, $D_{t}$, obtenido a partir de la primera ley de Fick relaciona el transporte en un sistema no equilibrado. Sin embargo, podemos definir el flujo de moléculas en un sistema en equilibrio siguiendo el movimiento de algunas moléculas "marcadas" mientras interaccionan con moléculas de la misma especie "no-marcadas". Aplicando la primera ley de Fick para esta situación especial, obtenemos la relación

$$
J_{i}^{*}=-D_{s}\left(\frac{\partial C^{*}}{\partial x_{i}}\right)
$$

donde el flujo de moléculas marcadas está representado por $J^{*}$, y $D_{s}$ representa el coeficiente de auto-difusión. Haciendo uso nuevamente del factor de corrección termodinámico, podemos relacionar el coeficiente de auto-difusión con respecto al coeficiente de difusión a traves de la relación 


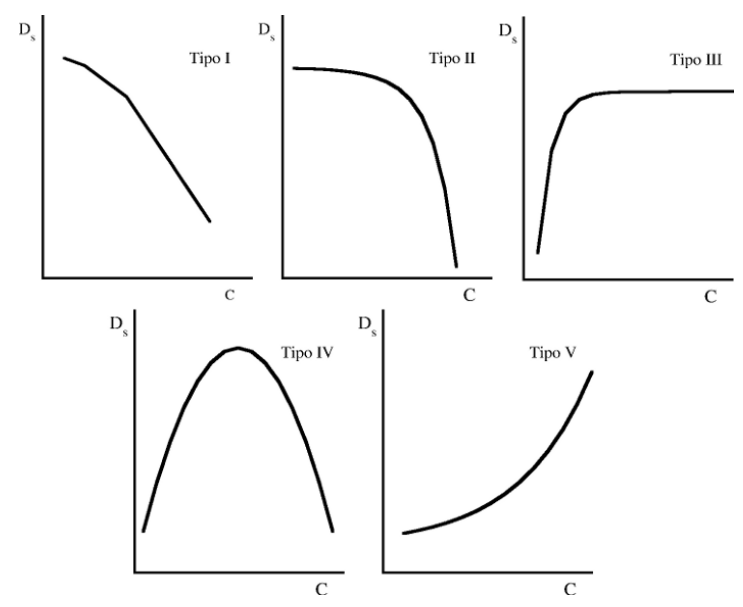

Figura 2.2: Tipos de dependencia de la difusividad con la concentración, de acuerdo a la nomenclatura propuesta por Kärger y Pfeifer. ${ }^{60}$

$$
D_{t}=D_{s}(0)\left(\frac{\partial \ln p}{\partial \ln q}\right)
$$

donde en este caso la dependencia de la presión se da con respecto a la concentración de la especie adsorbida en la zeolita. Esta ecuación tambien implica que a bajas concentraciones, el coeficiente de difusión de Fick es igual al coeficiente de auto-difusión. La implicación más importante de este hecho es que a bajas concentraciones los coeficientes de difusión medidos experimentalmente pueden ser comparados con coeficientes extraidos a partir de cálculos computacionales. En el capítulo IV, se hará extenso uso de esta aproximación para obtener los coeficientes de difusión de Fick de varios hidrocarburos a partir de cálculos de DM.

La figura 4 representa, de forma general, la dependencia de la auto-difusión con respecto a la concentración a partir de la clasificación de Kärger y Pfeifer. ${ }^{60}$ La dependencia de tipo I se da en zeolitas de poro grande en las cuales el adsorbato no presenta interacciones de tipo específico, tales como puentes de hidrógeno. En este caso, la difusividad disminuye de forma monotónica con respecto al aumento de la concentración, con el nivel de decrecimiento dado por el tamaño del adsorbato. En el segundo caso, se aprecia un nivel constante en el coeficiente de difusión hasta cierta concentración, a partir del cual el decaimiento de la difusividad es bastante acelerado. El tipo III, refleja la dependencia de la difusividad en dos tipos de moléculas polares, agua y amoníaco, en zeolita $\mathrm{NaX}$. El tipo IV es sin duda el más interesante de los casos, ya que existe un rango de concentraciones 
a lo largo del cual la difusividad aumenta, hasta alcanzar un máximo y luego disminuye, tal como en el caso I. Este tipo de comportamiento se da principalmente en zeolitas de poro pequeño con adsorbatos de dimensiones similares al tamaño de poro.

\subsubsection{Dependencia con la Temperatura}

Otro de los factores importantes que afectan el movimiento de especies adsorbidas en zeolitas es la temperatura. El proceso difusivo en los canales y poros de un material zeolítico depende principalmente de la interacción del adsorbato con las paredes del material. Por tanto, el movimiento del adsorbato a lo largo de la coordenada de difusión puede describirse como un movimiento activado, que se da entre sitios en los que existen las condiciones energéticas para la adsorción superficial de la moléculas. Este tipo de comportamiento puede describirse por medio del modelo de Arrhenius,

$$
D(T)=D_{0} \cdot \exp \left[-\frac{E_{a c t}}{R T}\right]
$$

en donde el término preexponencial, o coeficiente de difusión en el límite de temperatura, $D_{0}$, relaciona el coeficiente de difusión $D(T)$, en función de la temperatura $(T)$, siendo $E_{a c t}$ la energía necesaria para llevar a cabo el proceso de difusión. Esta energía de activación, $E_{a c t}$, representa la barrera energética que tendría que superar una molécula para efectual un salto entre dos sitios de adsorción, esto es, entre dos puntos donde la interacción de la molécula con el adsorbente es considerablemente más fuerte. A pesar de que este modelo solo incluye información simplificada acerca del proceso real de difusión, representa una excelente aproximación para la descripción del proceso, y su utilidad ha sido mostrada en una cantidad importante de estudios de difusión. Sin embargo, una nota de precaución es necesaria, debido a la dependencia de la concentración

con la temperatura, que, como vimos anteriormente, ejerce también una fuerte influencia en la constante de difusión. ${ }^{61}$

\subsubsection{Einstein y Movimiento Browniano}

Desde un punto de vista riguroso, en el sentido físico, podemos definir el desplazamiento medio al cuadrado (DMS) de las partículas del sistema por la ecuación

$$
\left\langle r^{2}(t)\right\rangle=\left\langle|\vec{r}(t)-\vec{r}(0)|^{2}\right\rangle=\frac{1}{N} \sum_{i=1}^{N}\left[\overrightarrow{r_{i}}(t)-\vec{r}_{i}(0)\right]^{2}
$$


donde $\vec{r}(t)$ representa el vector tridimensional de posición de la partícula $i$ en el momento $t$, y $N$ es el número total de partículas del sistema. Esta ecuación fué desarrollada por Einstein en sus estudios del movimiento Browniano, y representa el desplazamiento de las partículas en un tiempo $t$, representado por la distancia al cuadrado. ${ }^{31}$ Para tiempos lo suficientemente largos, puede demostrarse que el desplazamiento debido al "random walk" o "movimiento al azar" estará dado por la relación

$$
\left\langle\left|\vec{r}^{2}(t)\right|\right\rangle=(2 N) D_{s} t
$$

siendo $N$ en este caso la dimensionalidad de los canales de la zeolita. Esta relación es conocida com la relación de Einstein, y representa el hecho de que el desplazamiento al cuadrado de la partículas esta directamente relacionado con el tiempo, a través del coeficiente de auto-difusión. De forma alternativa, el coeficiente de difusión puede también obtenerse a partir de las relaciones de Green-Kubo, haciendo uso de la función de autocorrelación de la velocidad,

$$
D_{s}=\frac{1}{3} \int_{0}^{\infty}\langle\vec{v}(t) \cdot \vec{v}(t 0)\rangle
$$

Sin embargo, esta ecuación puede aplicarse solo en sistemas donde el nivel de restricción a la movilidad es suficientemente bajo como para garantizar que los cambios de velocidad se deben a desplazamientos de las moléculas más allá de los límites del poro, de otra forma estaremos unicamente verificando el tiempo entre colisiones intermoleculares adsorbato-adsorbente dentro del poro.

\subsection{Mecánica Estadística}

Las propiedades macroscópicas de un sistema son el producto de promedios de propiedades moleculares con respecto a la posición, el tiempo o una combinación de ambos. La termodinámica proporciona las relaciones fundamentales entre las propiedades macroscópicas de un sistema, sin embargo no proporciona los valores numéricos de dichas propiedades. Este tipo de información es obtenido a partir de propiedades microscópicas, tomando como base el promedio de dichas propiedades calculadas con modelos cuánticos o clásicos. La forma de calcular dichos promedios, que producirán los valores de las propiedades macroscópicas, está dada por la mecánica estadística.

Las propiedades básicas de un sistema cualquiera dependen, por lo general, de dos elementos: las posiciones y el momento de las partículas que lo conforman. 
Cada uno de los vectores de posición, $\overrightarrow{r_{i}}$, y momento, $\overrightarrow{p_{i}}$, tiene 3 componentes, en coordenadas cartesianas,

$$
\begin{gathered}
\overrightarrow{r_{i}}=\left(x_{i}, y_{i}, z_{i}\right)^{T} \\
\overrightarrow{p_{i}}=\left(p_{x i}, p_{y i}, p_{z i}\right)^{T}=\left(m_{i} v_{x i}, m_{i} v_{y i}, m_{i} v_{z i}\right)^{T}
\end{gathered}
$$

donde cada elemento de un vector corresponde a una dirección orthogonal. La dimensión de un sistema descrito de esta forma, tendrá por consiguiente dimension $(3 x 2 N) 6 N$. El vector de dimensión $6 N$ define, por lo tanto, el estado de un sistema de $\mathrm{N}$ partículas en el espacio de fase . Este espacio es por consiguiente un punto de referencia de coordenadas $\overrightarrow{r_{i}}$ y $\overrightarrow{p_{i}}$ para cada partícula del sistema. El espacio configuracional será por lo tanto un subespacio del espacio de fase, con $3 N$ elementos describiendo las posiciones de las moléculas, y el espacio de momentos, también con $3 \mathrm{~N}$ elementos correspondientes a la cantidad de movimiento de cada partícula.

El valor instantáneo de cualquier propiedad física, representada esa propiedad por $A\left(p^{N}(t), r^{N}(t)\right)$, estará definido como consecuencia de la interacción entre las partículas que conforman el sistema. Experimentalmente estos valores se miden durante un período dado de tiempo, y por tanto serán promedios calculados en el espacio temporal. Si el período de tiempo en que se toma la medida tiende a infinito, el valor de la propiedad $A$ tiende a su vez a tomar su valor real, o valor real promediado,

$$
A_{a v}=\lim _{\tau \rightarrow \infty} \frac{1}{\tau} \int_{\pi=0}^{\tau} A\left(p^{N}(t), r^{N}(t)\right) d t
$$

Por tanto, para calcular cualquier propiedad en un sistema de partículas, podemos simular dicho sistema, durante un período de tiempo suficientemente largo, basándo los cálculos en modelos de interacción inter e intra moleculares que describan lo más correctamente al sistema. A partir de los principios de la mecánica estadística, desarrollados por Boltzmann y Gibbs, el problema que tendríamos al intentar replicar un sistema de tamaño macroscópico, con número de particulas en el rango del número de Avogadro ( $10^{23}$ partículas $)$, se reduce a considerar simultaneamente un gran número de réplicas del sistema, o colectivos, de tamaño más manejable. De esta forma, los promedios en el tiempo son reemplazados por promedios obtenidos de un colectivo (ensemble), representado por:

$$
\langle A\rangle=\iint A\left(p^{N}, r^{N}\right) \rho\left(p^{N}, r^{N}\right) d p^{N} d r^{N}
$$


El símbolo $\langle\ldots\rangle$ representa el valor promedio del colectivo, o el valor promedio del observable A obtenido a partir del promedio sobre las réplica de sistema. La densidad de probabilidad, $\rho\left(p^{N}, r^{N}\right)$, representa la probabilidad de encontrar el sistema en una configuración determinada con valores de momento y posición dados por $p^{N}$ y $r^{N}$. Por tanto, el valor promedio del observable A está dado por la integral sobre todas las posibles configuraciones del sistema. De acuerdo a la hipótesis ergódica, postulado básico de la mecánica estadística, el promedio del colectivo es entonces igual al promedio a lo largo del tiempo. En condiciones de número de partículas $(\mathrm{N})$, volumen $(\mathrm{V})$ y temperatura $(\mathrm{T})$ constantes (colectivo NVT o colectivo canónico), la densidad de probabilidad estará representada por una distribución de Boltzmann

$$
\rho\left(p^{N}, r^{N}\right)=\frac{\exp \left(\frac{-E\left(p^{N}, r^{N}\right)}{k_{B} T}\right)}{Q}
$$

con energía $E\left(p^{N}, r^{N}\right)$ y donde $Q$ representa la llamada función de partición, $k_{B}$ es la constante de Boltzmann y Tes la temperatura. La función de partición puede representarse en términos del Hamiltoniano $\mathcal{H}$

$$
\mathcal{H}\left(p^{N}, r^{N}\right)=\sum_{i=1}^{N} \frac{\left|p_{i}\right|^{2}}{2 m_{i}}+V\left(r_{1}, \ldots, r_{N}\right)
$$

el cual representa la energía total del sistema: la cinética, calculada a partir del movimiento de las partículas y la potencial, con base en las interacciones entre las partículas del sistema. Para un sistema de $N$ partículas, idénticas, la función de partición para el colectivo canónico (NVT), estaría dada por

$$
Q_{N V T}=\frac{1}{N !} \frac{1}{h^{3 N}} \iint \exp \left(\frac{-\mathcal{H}\left(p^{N}, r^{N}\right)}{k_{B} T}\right) d p^{N} d r^{N}
$$

El factor $\frac{1}{N !}$ representa el hecho de que las partículas en el sistema son indistinguibles, y el factor $\frac{1}{h^{3 N}}$, donde $h$ es la constante de Planck, se requiere para asegurar que la función de partición sea igual al resultado mecano-cuántico para un sistema del tipo partícula-en-una-caja. ${ }^{48,49,51,53}$

\subsection{Dinámica Molecular}

La década de los años cuarenta del siglo pasado fué testigo de un inusitado interés en el campo de las ciencias de la computación, y especialmente de la simulación de sistemas a escala atomística. Este impulso inicial alrededor del 
uso de modelos y algoritmos para el modelamiento de sistemas reales ha seguido creciendo de manera sostenida a lo largo de los años. Sin embargo, los inicios en el campo del modelamiento y la simulación atomística, a pesar de su actual carácter beneficioso para la sociedad, tienen otro lado no tan admirable.

La motivación básica para el desarrollo del campo de la simulación fue la necesidad de entender los detalles de las explosiones nucleares. Con este fin se crearon las primeras máquinas de computación, en laboratorios nacionales de los Estados Unidos, especialmente en Los Alamos y en Livermore, en California. Como resultado puramente científico, resalta la utilización del método de Monte Carlo (MC) para la resolución de problemas basados en la mecánica estadística, descrito por los esposos Rosenbluth, Arianna y Marshal, y los esposos Teller, Augusta y Edward, así como por Metropolis en 1953, quien por esa época estaba a cargo de las únidades de computo en Los Alamos. ${ }^{62}$

Este primer intento por visualizar la dinámica de un sistema de partículas a nivel atomico fué sin embargo calificado como de no mucha utilidad por la comunidad de la mecánica estadística, y en especial los teóricos, ya que no representaba la evolución "real" de un sistema a nivel molecular, sino que eran "snapshots" o fotografías de configuraciones puntuales. La verdadera dinámica llegaría 4 años después, con el trabajo de dos pioneros, Berni Alder y Tom Wainwright ${ }^{63,64}$ quienes trabajaban en el laboratorio de Edward Teller por aquella época.

Su invención, la Dinámica Molecular, tomó principios conocidos de los procesos elementales de interacción entre dos partículas, y con la utilización de herramientas de cómputo disponibles, pudo resolver el problema de la interacción en sistemas de muchas partículas. Esto supuso la creación de verdaderas "películas", mostrando la evolución dinámica de sistemas moleculares complejos. Como podemos apreciar, el desarrollo de la DM fué, al igual que el del método de MC, llevado a cabo durante el desarrollo del proyecto Manhattan.

La técnica de la DM se basa en propagar en el tiempo las ecuaciones clásicas de movimiento, partiendo de un modelo matemático representativo de las fuerzas interatómicas existentes, y utilizando un conjunto adecuado de condiciones de frontera.

\subsubsection{Ecuaciones de movimiento de Newton}

La técnica de la DM se basa en la generación determinística de configuraciones de un sistema de partículas, las cuales son generadas a partir de la integración de las ecuaciones de movimiento de Newton. A nivel microscópico, todas las partículas $\{1 . . N\}$ que hacen parte del sistema tendrán exactamente 3 grados de libertad para desplazarse, con lo cual tendremos exactamente un conjunto de $3 \mathrm{~N}$ ecuaciones diferenciales acopladas de segundo orden por resolver. La segunda ley 
de Newton es descrita como

$$
F_{i}=\frac{d p_{i}}{d t}, i \in\{1 . . N\}
$$

donde $F_{i}$ representa la fuerza ejercida sobre la partícula $i$, como consecuencia de su movimiento, caracterizado por tener un momento $p$, a su vez está descrita por la ecuación

$$
\begin{gathered}
p_{i}=m_{i} v_{i} \\
v_{i}=\frac{d r_{i}}{d t}
\end{gathered}
$$

donde $m$ y $v$ representan masa y velocidad de la partícula $i$, respectivamente. La fuerza estará a su vez dada por la ecuación

$$
F_{i}=-\nabla V\left(\left\{r_{j}(t)\right\}\right)
$$

donde $V\left(\left\{r_{j}(t)\right\}\right)$ representa el potencial interatómico, y su dependencia con la posición. Al considerar un sistema aislado, donde no hay intercambio de masa o de energía con los alrededores, la integración de las ecuaciones de movimiento lleva a la conservación de la energía total del sistema,

$$
\begin{aligned}
& E_{t o t}=E_{c i n}+E_{p o t} \\
& E_{c i n}=\frac{1}{2} \sum m_{i} v_{i}^{2} \\
& E_{\text {pot }}=V\left(\left\{r_{j}(t)\right\}\right)
\end{aligned}
$$

donde $E_{c i n}$ y $E_{p o t}$ representan la energía cinética y potencial, repectivamente, del sistema.

La integración de las ecuaciones de movimiento genera una trayectoria que describe la posición, velocidad y aceleración de todas las partículas móviles que componen el sistema, y que varían con el tiempo. Para llevar a cabo esta tarea, se necesita de un integrador que reuna las siguientes características: Eficiencia, preservación de las restricciones de energía (conservación de la energía total) y reversibilidad en el espacio temporal, de acuerdo al teorema de Liouville. El algoritmo de Velocity-Verlet es sin duda el método más apropiado para llevar a cabo la integración de estas ecuaciones de movimiento en simulaciones atomísticas, y es el método utilizado en los cálculos presentados en este documento. El algoritmo se basa en una expansión de Taylor de las posiciones con respecto al tiempo, 


$$
\begin{gathered}
r_{i}(t+\Delta t)=r_{i}(t)+v_{i}(t) \Delta t+\left(\frac{1}{2}\right) a_{i}(t) \Delta t^{2} \\
F_{i}(t+\Delta t)=F_{i}\left(r_{i}(t+\Delta t)\right) \\
v_{i}(t+\Delta t)=v_{i}(t)+\frac{F_{i}(t+\Delta t)+F_{i}(t)}{2 m_{i}}
\end{gathered}
$$

El algoritmo así descrito es rápido, con requerimientos bajos de memoria, reversible, y muestra pocas fluctuaciones en el cálculo de la energía. La función de error del algoritmo, en posiciones y velocidades, es de orden $\mathcal{O}\left(\Delta t^{4}\right)$. Esta función de error, normalmente produce fluctuaciones de energía en el rango de $\frac{\Delta E_{t o t}}{E_{\text {tot }}}<10^{-5}$, valor adecuado para la mayoría de las aplicaciones de la DM. ${ }^{48,53,65-68}$

El paso de integración respecto al tiempo, $\Delta t$, es el parámetro más importante para la correcta implementación del método de simulación. Idealmente, debería ser lo suficientemenete grande para evitar el gasto innecesario de recursos de cómputo, pero no tan grande que la conservación de energía se vea afectada. Un compromiso adecuado entre gasto computacional y conservación de energía puede calcularse a partir de la ecuación

$$
\sigma_{E_{t o t}}^{2}=\left\langle E_{t o t}^{2}\right\rangle-\left\langle E_{t o t}\right\rangle^{2}
$$

donde las fluctuaciones de la energía, $\sigma_{E_{t o t}}^{2}$, deberían depender linealmente con $\Delta t^{2}$.

\subsubsection{Condiciones Periódicas de Frontera}

La aplicación de condiciones periódicas de frontera (PBC, por sus siglas en Inglés) en la celda de simulación se lleva a cabo principalmente para evitar el modelamiento de superficies, lo cual incrementaría en alto grado la complejidad del modelo y por supuesto impactaría la eficiencia computacional de los cálculos. Una de las ventajas de usar PBC es que se limita el tamaño de la celda de simulación, tamaño que sin embargo debe ser lo suficientemente grande para evitar correlaciones en la dinámica del sistema. Estas correlaciones aparecen debido a la limitación en el número de partículas necesarias para llevar a cabo un muestreo correcto dentro del colectivo que se esté utilizando.

En la definición de las PBC, la celda de simulación original es replicada a lo largo del espacio. Cuando una molécula en la celda original se desplaza, todas sus imágenes periódicas en las celdas cicundantes se desplazan exactamente en la misma forma. Si una molécula se desplaza más allá de los límites de la celda 


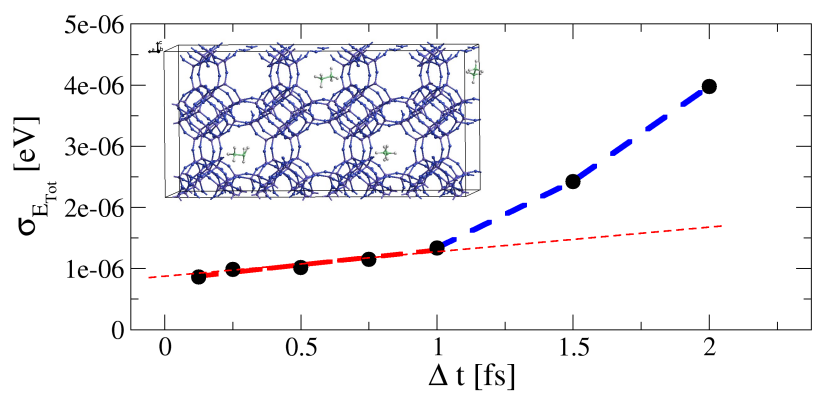

Figura 2.3: Desviación estandar de la energía total del sistema, $\sigma_{E_{t o t}}=\left(\left\langle E_{t o t}^{2}\right\rangle-\left\langle E_{t o t}\right\rangle^{2}\right)^{1 / 2}$, en función del paso de integración $\Delta t$. El sistema en este caso hace referencia a una celda de simulación compuesta por 800 átomos: 768 correspondientes a un material con estructura de tipo ITE (zeolita ITQ-3) con 4 moléculas de etano ( 8 átomos/molécula) adsorbidas. La temperatura de simulación es $300 \mathrm{~K}$, con todos los átomos del sistema móviles, en un colectivo microcanónico (NVE). La linea roja representa la dependencia lineal de $\sigma_{E_{t o t}}$ con respecto a $\Delta t$ para tiempos de integración menores de 1. La linea azul muestra la dependencia no lineal entre $\sigma_{E_{\text {tot }}}$ y $\Delta t$ que ocurre para pasos de integración mayores de 1 .

central, una de sus imágenes ingresará a la celda por el extremo opuesto. La ventaja de esta forma de modelar el sistema radica en que no es necesario almacenar las coordenadas y el momento de las imágenes, solo la celda central es necesaria, ya que las imágenes pueden ser obtenidas a través de operadores de translación. Los límites entre celdas no tienen ningún significado físico, unicamente la forma y orientación son fijas, y dependerán de las características de la celda unidad del sólido que se modela.

Normalmente se utiliza la convención de la imagen mínima, donde se define la distancia entre dos partículas como la distancia más corta entre sus imágenes periódicas. Esta definición se complementa con la definición de un cut-off, que es la distancia máxima a la que se considera que dos partículas interactúan a traves del potencial. El cut-off es nomalmente aplicado a una distancia igual o menor a la mitad de la longitud de la celda, en su parámetro de red más corto, para evitar inconsistencias con la definición de la imagen mínima. El cut-off, debe ser también lo suficientemente grande para evitar el alto grado de correlación que se da entre partículas cercanas entre sí. La función de distribución de distancias entre partículas, $g(r)$, define la probabilidad de encontrar dos partículas a una distancia $r$ una de otra, relativa a la probabilidad esperada para una distribución totalmente aleatoria a la misma densidad.

En cristalografía se define la celda unidad como la porción mínima del sólido 


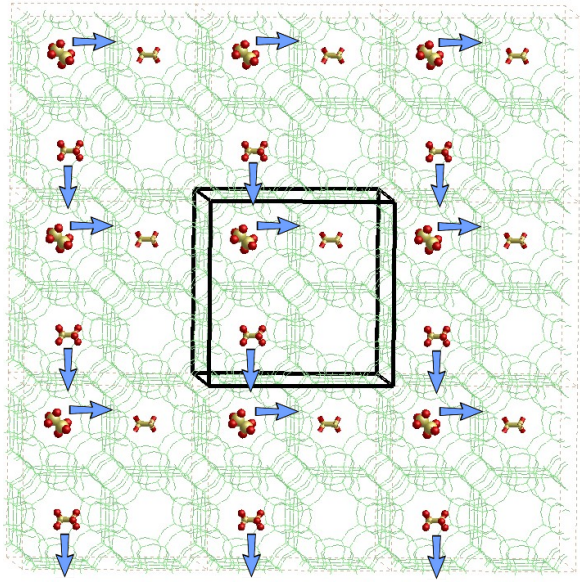

Figura 2.4: Representación esquemática de las condiciones periódicas de frontera.

que mantiene sus características topológicas intactas y se describe por medio de los parámetros de la celda y las coordenadas fraccionales de los átomos que la componen. Este conjunto de coordenadas define lo que se conoce como un espacio adimensional ortonormal o espacio-S. La matriz $\mathrm{H}$ define las operaciones de transformación desde el espacio-S al espacio real o espacio-R, en la forma:

$$
F=\left(\begin{array}{ccc}
a & b \cos (\gamma) & c \cos (\gamma) \\
0 & b \sin (\gamma) & c \varsigma \\
0 & 0 & c\left(1-\cos ^{2} \beta-\varsigma^{2}\right)
\end{array}\right)
$$

donde

$$
\varsigma=\frac{\cos (\alpha)-\cos (\gamma) \cos (\beta)}{\sin (\gamma)}
$$




\title{
Capítulo 3
}

\section{Campos de Fuerza para el Estudio de Polimorfos Silíceos}

\begin{abstract}
A pesar del uso continuo de metodologías basadas en campos de fuerza para el estudio de polimorfos de $\mathrm{SiO}_{2}$, existen pocos trabajos que revisen en detalle la literatura en este tema. En esta sección se presenta un análisis de varios modelos utilizados para la predicción de propiedades estructurales y dinámicas en materiales silíceos. Los potenciales han sido seleccionados entre los mas utilizados en técnicas de simulación molecular, y se analiza su nivel de precisión y transferibilidad en la predicción de propiedades mecánicas, estructurales y dinámicas de polimorfos silíceos.
\end{abstract}

El uso de métodos computacionales para el estudio de materiales silíceos ha sido vital para el desarrollo del campo de los materiales de diseño. Los trabajos pioneros de Catlow, Demontis, Jackson, Vessal y otros, ${ }^{67,69-73}$ sobre el modelamiento de las fuerzas físicas que aparecen como consecuencia de la interacciones mecano-cuánticas entre electrones y núcleos atómicos, han sido básicos para el desarrollo de metodologías computacionales y por supuesto, para la comprensión de procesos que ocurren a escala molecular en dichos materiales. Este tipo de procesos hace uso de escalas de tamaño y tiempo que exceden en términos de capacidad computacional los recursos requeridos por modelos cuánticos. Sin duda alguna, las técnicas de simulación basadas en potenciales analíticos han demostrado su capacidad para el estudio de sistemas en diferentes campos de las ciencias, desde la determinación de cambios de fase en materiales cristalinos a partir de campos de fuerza reactivos, ${ }^{74,75} \mathrm{o}$ el estudio de interfases reactivas solido-líquido ${ }^{76,77}$ pasando por la determinación estructural de proteínas en combinación con técnicas experimentales cristalográficas, ${ }^{78}$ hasta la predicción de fases interme- 
dias en procesos de síntesis de estructuras cristalinas. ${ }^{79,80}$ Esta capacidad para simular de manera confiable y cuantitativa la fenomenología de procesos moleculares depende, en primera instancia, de los modelos utilizados para representar las interacciones atómicas.

Tal como se definió en el capítulo anterior, la técnicas de simulacion molecular se basan en la representación de las interacciones entre partículas atómicas a través de campos de fuerza, que se definen a través de funciones matemáticas adecuadamente parametrizadas. En el ámbito específico de la dinámica molecular el campo de fuerza es el factor determinante para evaluar la calidad de los resultados obtenidos. En primer lugar, las fuerzas físicas que rigen el comportamiento tanto estático como dinámico de un sistema, deben estar definidas en el campo de fuerza, de forma tal, que representen adecuadamente el modelo real.

En el caso de propiedades estáticas en materiales cristalinos podemos nombrar algunas propiedades de interés como las constantes de red, las distancias interatómicas, angulos de enlace, el módulo de Young y otras. Como ejemplos de propiedades dinámicas podemos destacar las constantes vibracionales y los coeficientes de difusión como los mas importantes. En este sentido, un aspecto muy importante en el campo de la simulación molecular es la influencia de la flexibilidad de la red en la dinámica y, como consecuencia lógica de la flexibilidad de la red, la variación dinámica del tamaño de poro. Algunos estudios a este respecto pueden ser encontrados en la literatura, ${ }^{81-87}$ aunque en ocasiones el factor de flexibilidad de la red no es tenido en cuenta. ${ }^{88,89}$ El modelamiento dinámico de un sistema se hace imprescindible cuando se estudian fenómenos de transferencia de masa como la adsorción y difusión en materiales porosos. El campo de fuerza diseñado para modelar un sistema dinámico debe ser, por lo tanto, capaz de modelar las fuerzas interatómicas de forma tal que permita la inclusión de grados de libertad asociados a la dinámica intramolecular y a la fenomenología que se produce debido al movimiento relativo intermolecular.

El propósito principal del presente capítulo es el de presentar una selección amplia de funciones de energía potencial para materiales silíceos y su utilización en el cálculo y posterior comparación con propiedades estructurales, dinámicas y mecánicas aplicado a un conjunto de polimorfos de sílica. Los materiales utilizados en la comparación se dividen en dos grupos: polimorfos de alta densidad y bajo orden estructural y polimorfos de baja densidad con alta cristalinidad. En el grupo de los materiales de alta densidad tenemos las dos variedades de quarzo, $\alpha$ y $\beta$, así como la coesita. Los polimorfos de alta densidad seleccionados son las zeolitas formadas por pura sílice con estructuras de tipo LTA, CHA, MWW y SAS.

Las propiedades estructurales calculadas son los parámetros de red, volumenes, longitudes de enlace $\mathrm{SiO}$ y $\mathrm{SiSi}$, y por último los ángulos de enlace $\widehat{\operatorname{SiOS} i}$ 
y $\widehat{O S i O}$. Las propiedades mecánicas evaluadas son las constantes de elasticidad y el módulo Bulk. Estas dos propiedades se seleccionan por ser extremadamente sensibles a los campos de fuerza, ya que se definen en función de las derivadas segundas de la energía potencial con respecto a las componentes de la carga mecánica. Complementariamente, las constantes dieléctricas estáticas y a altas frecuencias tambien fueron calculadas para el conjunto de potenciales. Finalmente, la densidad de estados vibracionales a presión cero se calcularon para evaluar la capacidad de algunos de los potenciales con miras a su utilización en cálculos computacionales de simulación molecular.

\subsection{Un Vistazo a la Literatura}

El campo de la simulación computacional aplicada a materiales silíceos es un ejemplo de una disciplina extremadamente exitosa y, además, es un área de investigación muy activa en la actualidad, debido, principalmente, a los continuos desarrollos en el campo de los materiales y de las ciencias de la computación. Ejemplos de este desempeño exitoso a lo largo del tiempo son los trabajos pioneros de Sanders et al., ${ }^{69}$ que muestran las aplicaciones de técnicas computacionales basadas en campos de fuerza interatómicos en el área de los materiales, o el desarrollo de campos de fuerza para modelos moleculares mecanísticos de Smirnov et al. ${ }^{90}$ que han servido de base a muchos estudios en el area de materiales, y mas especificamente en materiales zeolíticos.

El desarrollo de campos de fuerza capaces de predecir de forma precisa y eficiente las propiedades estructurales y dinámicas de materiales silíceos se inicia con el desarrollo de modelos muy sencillos. Estos potenciales, desarrollados siguiendo el modelo de Born-Mayer ${ }^{91}$ fueron usados para la predicción de propiedades estructurales relevantes de polimorfos de silicio. Sin embargo, la precision de las propiedades calculadas era limitada, debido a que el campo de fuerza mostraba algunas carencias debido a una incompleta representación de las interacciones $\mathrm{Si}-\mathrm{O} .{ }^{92}$

La inclusión de términos que representan interacciones entre tres cuerpos, SiOSi ó OSiO, ${ }^{93,94}$ y mas adelante la adición de formas funcionales para caracterizar los efectos de polarización, han permitido un mejoramiento cualitativo en la modelización de las estructuras cristalinas. Ejemplos de campos de fuerza que integran términos para evaluar la polarizabilidad son los potenciales basados en iones core-shell de Schröder y Sauer ${ }^{95}$ y Gale,${ }^{96}$ el modelo de la carga variable en los potenciales de Garofalini ${ }^{97}$ y el modelo de la igualación de cargas de Rappé y Goddard. ${ }^{98,99}$

Algunos esfuerzos destacados sobre la comparación de potenciales para materiales silíceos han sido publicados en los últimos años. Para citar algunos ejem- 
plos, tenemos los trabajos de Huang et al., ${ }^{100}$ Herzbach et. al. ${ }^{101}$ y mas recientemente por Zwijnenburg et al. ${ }^{102}$ y Muralidharan et al. ${ }^{103}$ Estos autores han comparado potenciales ampliamente utilizados, tales como el potencial de van Beest et al. ${ }^{104}$ y Tsuneyuki et al. ${ }^{105}$ con potenciales recientemente desarrollados, los cuales incluyen representaciones mas sofisticadas de las cargas atómicas o parametrizaciones alternativas de potenciales clásicos que presentan mejoras en las predicción de propiedades termomecánicas, estructurales y dinámicas de materiales basados en óxido de silicio.

En el trabajo de Huang et al., ${ }^{100}$ se compara la capacidad de los potenciales BKS, TTAM, Sanders et al. ${ }^{69}$ y Kramer et al. ${ }^{106}$ para el cálculo de densidades de estado vibracionales, en configuraciones minimizadas de materiales silíceos amorfos.

Herzbach et al. ${ }^{101}$ van un paso mas allá y comparan propiedades estructurales, termomecánicas y dinámicas de polimorfos de silica usando los potenciales clásicos de van Beest et al. ${ }^{104}$ y Tsuneyuki et al. ${ }^{105}$ con los potenciales basados en el principio de igualación de cargas de Demiralp et al. ${ }^{107}$ y el modelo de polarización dipolar de Tangney et al. ${ }^{108}$ Este último potencial está parametrizado a partir de datos obtenidos con técnicas ab-initio, únicamente, e incluye además el esquema de polarización dipolar de Madden y Wilson. ${ }^{109}$

Mas recientemente, Zwijnenburg et al. ${ }^{102}$ comparan datos termoquímicos experimentales de materiales silíceos que presentan anillos de 3 unidades de $\mathrm{SiO}_{2}$ con predicciones hechas a partir de los potenciales Sanders et al., ${ }^{69}$ van Beest et al., ${ }^{104}$ Sastre et al. ${ }^{110}$ y de predicciones con modelos períodicos a partir de cálculos de la teoría del funcional de la densidad (DFT).

En esta misma línea, el trabajo de Muralidharan et al. ${ }^{103}$ muestra propiedades energéticas y estructurales de materiales puramente silíceos calculadas a partir de potenciales de tipo átomo embebido (EAM). Este tipo de modelo, dependiente del medio a través de cargas variables, tiene la particularidad de estar parametrizado con datos de energía y estructura obtenidos a partir de técnicas DFT aplicadas a pequeños clusters compuestos de unidades silíceas. La comparación se centra en las diferencias de este nuevo esquema con los modelos de Flikkema y Bromley, ${ }^{111}$ y con los omnipresentes modelos van Beest y Tsuneyuki.

\subsection{Seleccion de los Campos de fuerza}

La selección de los campos de fuerza se ha llevado a cabo partiendo de su aplicabilidad a materiales zeolíticos. Los potenciales seleccionados han sido agrupados en varios conjuntos. Primero, un conjunto de potenciales clásicos, con términos de interacción entre dos y tres partículas de tipo Born-Mayer, con iones rígidos, entre los que se destacan los potenciales de van Beest et al. y Tsuneyuki et 
al. Este conjunto inicial es complementado con los potenciales derivados o parametrizados por Auerbach et al., ${ }^{112}$ Jaramillo y Auerbach, ${ }^{113}$ Vessal, ${ }^{93}$ Jackson y Catlow $^{72}$ y los potenciales de Pedone et al. ${ }^{114,115}$

Un segundo grupo está integrado por potenciales con cargas formales, de tipo core-shell con términos de interacción entre dos y tres partículas. Los potenciales son: Sanders et al., ${ }^{69}$ Gale, ${ }^{96}$ Sastre y Corma, ${ }^{116}$ Schröder y Sauer, ${ }^{95}$ Sierka y Sauer ${ }^{117}$ y el potencial de Jackson and Catlow. ${ }^{72}$ Tres potenciales especiales, parametrizados exclusivamente para ser utilizados en cálculos de mecánica molecular, también han sido escogidos. Esta categoría esta compusta por los potenciales de Demontis et al., ${ }^{70,71}$ Smirnov et al. ${ }^{90}$ y Sastre y Corma. ${ }^{116}$

Potenciales que usan modelos sofisticados para la inclusión de efectos de polarizabilidad, han sido agrupados en el último grupo. Entre estos están los potenciales basados en el modelo de igualación de cargas de Rappé y Goddard, ${ }^{98}$ específicamente los potenciales de Demiralp et al. ${ }^{107}$ y Sefcik et al. ${ }^{99}$ Algunos otros potenciales desarrollados recientemente no han sido incluidos en este trabajo, debido principalmente a que su implementación en códigos estándar de simulación aún no ha sido llevada a cabo, y por tanto requerirían de su programación directa en códigos existentes. Algunos de estos potenciales son los desarrollados por Tilocca et al. ${ }^{118}$ o el modelo de la fluctuación de cargas de Tangney y Scandolo, ${ }^{108,119}$ Olano y Rick, ${ }^{120}$ y Garofalini et al. ${ }^{97,121-123}$

La tabla 3.1 muestra las principales características de los potenciales seleccionados. La clasificación se da con respecto a:

- Método de Parametrización

- Estructura usada para la parametrización

- Si la polarizabilidad es incluída explicitamente via el modelo Shell, igualación de carga o fluctuación de carga

- La carga de las partículas, sea esta formal o parcial

- La aplicación del campo de fuerza, siendo las mas conocidas: cálculos para la determinación de parámetros estructurales, minimización de energía libre, minimización de estructuras, mecánica molecular y dinámica molecular

Los parámetros para cada uno de los potenciales descritos en la tabla 3.1 se incluyen en el Apéndice A, tabla A1. En las siguientes secciones se presentan las formas funcionales utilizadas por los potenciales seleccionados, y se describen las estructuras silíceas escogidas para la evaluación de propiedades. Los resultados obtenidos son presentados en el Apéndice A, y son discutidos en la sección correspondiente en el presente capítulo. 


\begin{tabular}{|c|c|c|c|}
\hline Campo de Fuerza & Parametrización (Modelo) & Polarizabilidad & Applicación \\
\hline Sanders et al. ${ }^{69}$ (SLC) & Empirico $($ Cuar $z o-\alpha)$ & CF / MS & Estructura de Red \\
\hline Schroeder and Sauer ${ }^{95}$ (SS96) & $\mathrm{Ab}$ initio $\left(\mathrm{SiO}_{4}, \mathrm{AlO}_{4}\right)$ & CF / MS & Estructura de Red \\
\hline \multirow[t]{2}{*}{ Sierka and Sauer ${ }^{117}$ (SS97) } & Ab initio & CF / MS & Estructura de Red \\
\hline & Anillos 4-6, $\mathrm{SiO}_{4 / 2} \mathrm{AlO}_{4}$ & & Reactividad \\
\hline Gale $^{96}$ (Gale) & Empirico (Cuar zo - $\alpha)$ & CF / MS & Min. Energía Libre \\
\hline Sastre and Corma ${ }^{116}(\mathrm{SCI})$ & Empirico $($ Cuar $z o-\alpha)$ & CF / MS & Est. Red y Estabilidad \\
\hline Sastre and Corma ${ }^{116}{ }_{(S C 2)}$ & Empirico (Cuar $z o-\alpha)$ & $\mathrm{CF} / \mathrm{MS}$ & Est. Red y Estabilidad \\
\hline Jackson and Catlow ${ }^{72}(\mathrm{JCI})$ & Empirico (Cuar zo $-\alpha)$ & $\mathrm{CF} / \mathrm{MS}$ & Min. Energía Libre \\
\hline Jackson and Catlow ${ }^{72}(\mathrm{JC} 2)$ & Empirico (Cuar $z o-\alpha)$ & $\mathrm{CF} / \mathrm{IR}$ & Min. Energía Libre \\
\hline Demontis et al. ${ }^{70}(D S)$ & Empirico (Natrolita, Zeolitas) & $\mathrm{CF} / \mathrm{IR}$ & Dinámica Molecular \\
\hline Jaramillo and Auerbach $^{113}(\mathrm{JA})$ & $\begin{array}{l}\text { Ab initio / Empirico } \\
(\text { Cuar zo }-\alpha)\end{array}$ & $\mathrm{CP} / \mathrm{IR}$ & Dinámica Molecular \\
\hline Auerbach et al. ${ }^{112}$ (AHCM) & $\begin{array}{l}\text { Ab initio / Empirico } \\
(\text { Cuar } z o-\alpha)\end{array}$ & $\mathrm{CP} / \mathrm{IR}$ & Dinámica Molecular \\
\hline Van Beest et al. ${ }^{104}(B K S)$ & $\begin{array}{l}\text { Ab initio / Empirico } \\
(\text { Cuarzo }-\alpha)\end{array}$ & $\mathrm{CP} / \mathrm{IR}$ & Dinámica Molecular \\
\hline Vessal $^{93}$ (Vessal) & $\begin{array}{l}\text { Empirico } \\
(\text { Cuarzo }-\alpha)\end{array}$ & $\mathrm{CF} / \mathrm{IR}$ & Dinámica Molecular \\
\hline Pedone et al. ${ }^{114}$ (PMM06) & $\begin{array}{l}\text { Empirico } \\
\text { (Estructuras Variadas) }\end{array}$ & $\mathrm{CP} / \mathrm{IR}$ & Dinámica Molecular \\
\hline Pedone et al. ${ }^{115}$ (PMM08) & DFT Periódico & $\mathrm{CP} / \mathrm{MS}$ & Estructura de Red+DM \\
\hline Tsuneyuki et at. ${ }^{105}$ (TAM) & Ab initio (Cuarzo $-\alpha)$ & $\mathrm{CP} / \mathrm{IR}$ & DM \\
\hline Smirnov and Bougeard ${ }^{90}$ (SB) & & $\mathrm{CP} / \mathrm{IR}$ & Mecánica Molecular \\
\hline Demiralp et al. ${ }^{107}$ (DCG) & & $\operatorname{IgQ} / \mathbf{I R}$ & Dinámica Molecular \\
\hline Sefcik et al. ${ }^{99}$ (SDCG) & & IgQ / IR & Dinámica Molecular \\
\hline Tangney and Scandolo ${ }^{108}$ (TS) & DFT Periódico (LDA-GGA) & $\mathrm{CP} / \mathrm{PD}$ & Dinámica Molecular \\
\hline Ma and Garofalini ${ }^{97}$ (MG) & & IgQ-EEM & Dinámica Molecular \\
\hline Tilocca et al. ${ }^{118}$ (TLC) & Basado en el potencial SLC & CF / MS & DM-MS Adiabatico \\
\hline Olano and Rick ${ }^{120,124}(\mathrm{OR})$ & & FQ & Dinámica Molecular \\
\hline$=$ Carga Formal, MS=Modelo & $\mathrm{CP}=$ Carga Parcial, $\mathrm{IR}=\mathrm{Ión}$ & & \\
\hline
\end{tabular}

Tabla 3.1: Campos de fuerza, acrónimo, método de parametrización, base estructural para su parametrización, polarizabilidad y aplicaciones. 


\begin{tabular}{lccccccc}
\hline Estructura & $\mathbf{a}[\AA]$ & $\mathbf{b}[\AA]$ & $\mathbf{c}[\AA]$ & $\alpha[\mathbf{r a d}]$ & $\beta[\mathbf{r a d}]$ & $\gamma[\mathbf{r a d}]$ & $\mathbf{V o l}\left[\AA^{3}\right]$ \\
\hline Cuarzo- $\alpha^{\mathbf{1 2 5}}$ & 4.916 & 4.916 & 5.405 & 90.00 & 90.00 & 120.00 & 113.13 \\
${\text { Cuarzo- } \beta^{\mathbf{1 2 5}, \mathbf{1 2 6}}}$ & 4.997 & 4.997 & 5.458 & 90.00 & 90.00 & 120.00 & 118.01 \\
Coesita $^{\mathbf{1 2 7}}$ & 7.136 & 12.383 & 7.186 & 90.00 & 120.38 & 90.00 & 547.82
\end{tabular}

Tabla 3.2: Cuarzo $\alpha, \beta$ y coesita: parámetros de red y volúmenes de celda unidad.

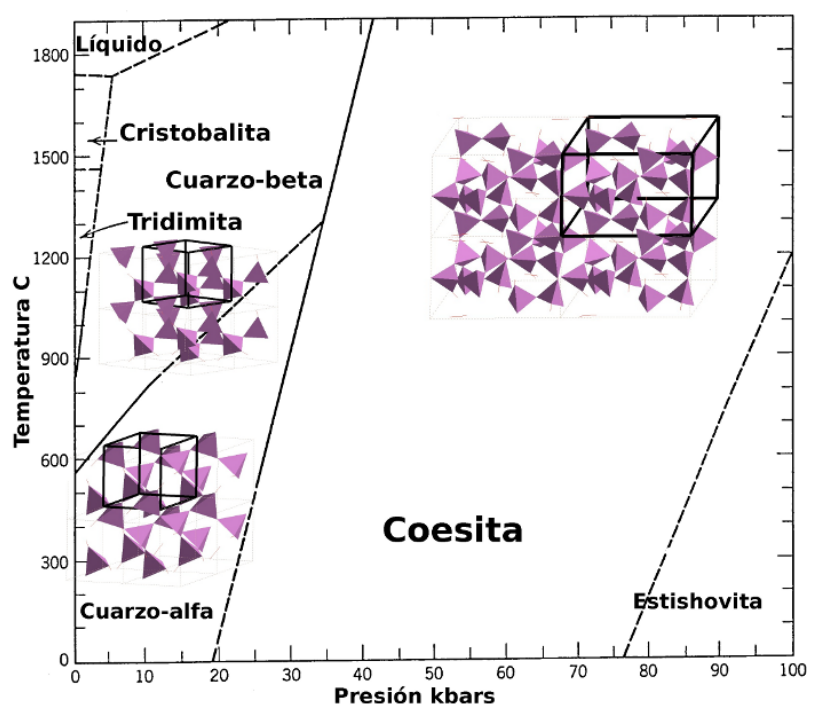

Figura 3.1: Diagrama de fases del cuarzo. La representación poliédrica de las estructuras del cuarzo- $\alpha$, cuarzo- $\beta$ y coesita se muestra en las regiones respectivas.

\begin{tabular}{lccccccc}
\hline Zeolite & $\mathbf{a}[\AA]$ & $\mathbf{b}[\AA]$ & $\mathbf{c}[\AA]$ & $\alpha[\mathbf{r a d}]$ & $\beta[\mathbf{r a d}]$ & $\gamma[\mathbf{r a d}]$ & $\left.\mathbf{V o l}_{[\AA} \AA^{3}\right]$ \\
\hline ITQ-29 $^{\mathbf{1 2}}$ & 11.846 & 11.846 & 11.846 & 90.00 & 90.00 & 90.00 & 1662.53 \\
SSS-73 $^{\mathbf{1 0}}$ & 14.104 & 14.104 & 10.187 & 90.00 & 90.00 & 90.00 & 2026.50 \\
Si-CHA $^{\mathbf{1 2 8}}$ & 13.675 & 13.675 & 14.767 & 90.00 & 90.00 & 120.00 & 2391.54 \\
ITQ-1 $^{\mathbf{1 2 9}}$ & 14.208 & 14.208 & 24.945 & 90.00 & 90.00 & 120.00 & 4361.00
\end{tabular}

Tabla 3.3: Detalles estructurales de los polimorfos silíceos de baja densidad y alta cristalinidad con estructuras de tipo LTA, CHA, MWW and SAS. 


\subsection{Modelos de Polimorfos Silíceos}

Tres estructuras polimorfas silíceas de alta densidad fueron seleccionadas para la evaluación de los FF: cuarzo $\alpha$ y $\beta$ y coesita. El polimorfo $\alpha$ es la fase termodinamicamente estable del cuarzo a condiciones normales, y es una estructura compuesta por unidades tetraédricas de $\mathrm{SiO}_{4}^{-4}$ unidas por los vértices. La celda unidad del cuarzo $\alpha$ presenta simetría espacial trigonal $P 3_{1} 21$. Además, representa una fuente de información básica para la parametrización de muchos potenciales interatómicos, debido a que es una de las estructuras mas estudiadas, tanto por su utilidad como por su ubicuidad en la naturaleza.

El cuarzo $\beta$ es una fase que se presenta a temperaturas superiores a los 847 $\mathrm{K}$. Se produce como consecuencia de un cambio de fase del cuarzo $\alpha$, que ocurre en el rango de temperaturas entre 846 a $847,1 \mathrm{~K} .{ }^{130}$ La estructura del cuarzo $\beta$ presenta una celda unidad hexagonal, con simetría de grupo $P 6_{1} 22$.

Por último, la coesita es la fase que se genera a partir del cuarzo $\alpha$ a altas presiones, y presenta una estructura muy particular que tiene dos unidades tetraédricas no equivalentes. Esta característica convierte a la coesita en un especimen excelente para evaluar la transferabilidad de los campos de fuerza, especialmente aquellos parametrizados a partir de la estructura del cuarzo $\alpha .{ }^{131}$ La coesita tiene una celda unidad e tipo monoclínico-beta, compuesta por 48 átomos, con simetría de grupo $C 2 / c$. La tabla 3.2 sumariza los parámetros estructurales para estas tres estructuras, y la figura 3.1 muestra el diagrama de fases del cuarzo.

En el grupo de los polimorfos de baja densidad y alto nivel de ordenamiento estructural se han seleccionado 4 tipos de red compuestos por sus variantes puramente silíceas: LTA, CHA, MWW and SAS. La estructura silícea LTA (zeolita ITQ-29) consiste en un arreglo cúbico de celdas tipo sodalita conectadas entre sí por sus caras cuadradas, con lo que se forman estructuras dobles de anillos de 4 unidades tetraédricas de $\mathrm{SiO}_{4 / 2}$. La celda unidad está compuesta por 72 átomos con simetría de grupo $P m 3 m .^{12}$

La estructura de tipo CHA presenta una celda unidad romboédrica de 36 átomos, con simetía espacial $R 3 m$ y una distribución de cuatro ángulos cristalográfi$\cos \widehat{\operatorname{SiOS} i}$ diferentes. ${ }^{128}$

La zeolita ITQ-1, es el equivalente estructural de tipo MWW puramente silíceo. Presenta una topología idéntica a la MCM-22, unidad de celda hexagonal y simetría espacial $P 6 / \mathrm{mm}$. La estructura forma un sistema de canales bidimensional conectado através de anillos de 10 unidades tetraédricas. ${ }^{129}$

Por último, la zeolita SSZ-73 es el equivalente silíceo de estructura de red SAS, con canales unidimensionales formados por anillos de 8, 6 y 4 unidades de $\mathrm{SiO}_{4 / 2}$, y con simetría espacial $14 / \mathrm{mmm} .{ }^{10}$ La tabla 3.3 muestra los parámetros de red y volúmenes de las zolitas seleccionadas. El conjunto de sólidos porosos 
seleccionado es un grupo lo suficientemente representativo estructuralmente para evaluar las carácterísticas de lo potenciales, además, ya que la mayoría de potenciales ha sido parametrizado a partir de información estructural y energética del cuarzo $\alpha$, tendremos la oportunidad de evaluar ampliamente su transferabilidad.

\subsection{Propiedades Calculadas}

Tal como se mencionó en la introducción, hemos utilizado los campos de fuerza seleccionados aplicados a los polimorfos de sílica descritos en la sección 3.3 para el cálculo de propiedades estructurales, mecánicas y dinámicas. Para llevar a cabo la determinación de dichas propiedades hemos utilizado el código GULP (General Utility Lattice Program), ${ }^{132,133}$ desarrollado para la utilización de técnicas de dinámica de red a través de la resolución de modelos analíticos. Este programa permite la utilización e implementación de campos de fuerza partiendo de formas funcionales estandar para la definición de interacciones intra e intermoleculares.

En todos los casos, las estructuras experimentales reportadas han sido utilizadas como punto de partida para los cálculos. La optimización de las estructuras es llevada a cabo a través de un proceso de minimización de energía a presión constante, incluyendo todos los grados de libertad internos dentro de las celdas de simulación. Para llevar a cabo el análisis de la conectividad estructural, longitudes de enlace, ángulos entre triadas, etc, hemos utilizado el código ZeoTsites. ${ }^{134}$ Los resultados de los cálculos se presentan de forma extensiva en el Apéndice I.

\subsubsection{Propiedades Estructurales}

Uno de los cálculos computacionales mas comunes en el estudio de materiales es la optimización estructural. Una vez se han definido los diferentes términos de la función de energía potencial, descritos anteriormente, es necesario tratar de hallar la estructura que represente un mínimo global en dicha superficie de energía. Por tanto, el desafío que representa la optimización de una estructura es nada mas que la aplicación de una metodología eficiente de optimización. Algunos métodos utilizan técnicas basadas en algoritmos genéticos, Monte Carlo o dinámica molecular para la ubicación de mínimos globales, mientras que otras técnicas exploran analíticamente la matriz de derivadas direccionales de la función de energía potencial para encontrar mínimos locales. El código utilizado para nuestros cálculos es del segundo tipo, y está incluido en el programa de simulación descrito anteriormente.

Los métodos de minimización de energía pueden describirse a partir de la 

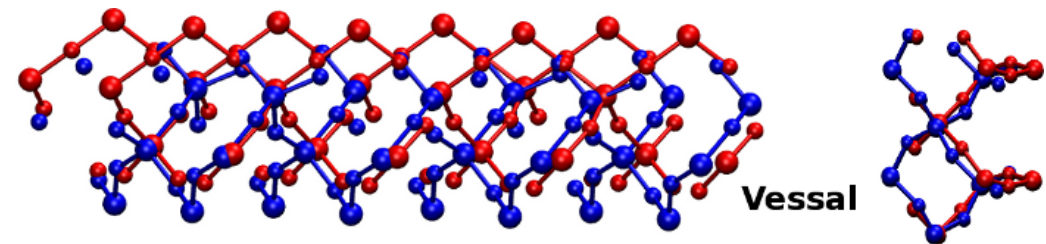

[010]

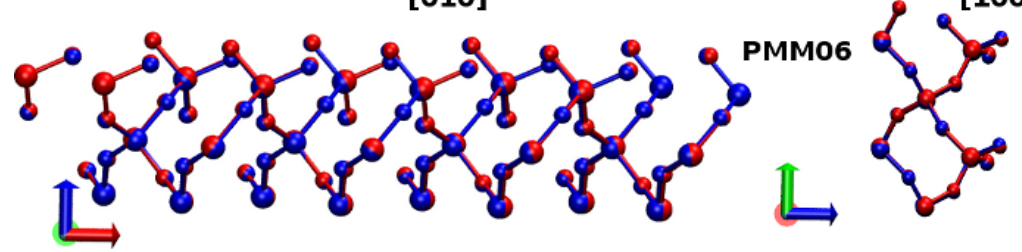

Figura 3.2: Representación de la estructura experimental del Cuarzo- $\alpha$, en color azul, en comparación con estructuras optimizadas utilizando los campos de fuerza Vessal (arriba) y PMM06 (abajo), superposición en rojo. Adicionalmente, la representación de las superficies [010] (izquierda) y [100] (derecha) permiten observar mas claramente el nivel de coincidencia de las estructuras minimizadas respecto a la estructura experimental.

expansión de la energía potencial en series de Taylor

$$
V(r+\delta r)=V(r)+\frac{\partial V}{\partial r} \delta r+\frac{1}{2 !} \frac{\partial^{2} V}{\partial r^{2}}(\delta r)^{2+\cdots}
$$

donde el segundo término de la derecha representa el vector gradiente de la función de energía potencial, y el tercer término representa la matriz de segundas derivadas de $V(r)$, o matriz Hessiana $H$, la cual define la curvatura de la superficie de energía potencial en términos de sus variables,

$$
H(V(r))=\left[\begin{array}{cccc}
\frac{\partial^{2} V}{\partial r_{1}^{2}} & \frac{\partial^{2} V}{\partial r_{1} \partial r_{2}} & \cdots & \frac{\partial^{2} V}{\partial r_{1} \partial r_{n}} \\
\frac{\partial^{2} V}{\partial r_{2} \partial r_{1}} & \frac{\partial^{2} V}{\partial r_{2}^{2}} & \cdots & \frac{\partial^{2} V}{\partial r_{2} \partial r_{n}} \\
\vdots & \vdots & \ddots & \vdots \\
\frac{\partial^{2} V}{\partial r_{n} \partial r_{1}} & \frac{\partial^{2} V}{\partial r_{n} \partial r_{2}} & \cdots & \frac{\partial^{2} V}{\partial r_{n}{ }^{2}}
\end{array}\right]
$$

Debido al comportamiento armónico del sistema en cercanías del mínimo, la expansión de Taylor suele truncarse después del segundo término. Un método muy eficiente de minimización, utilizado en GULP, el es de los gradientes conjugados, que utiliza el algoritmo de Newton-Raphson para calcular los desplazamientos consecutivos en la coordenada de exploración de la función de energía potencial.

$$
\Delta r=-\alpha H^{-1} \nabla V(r)
$$


Este método involucra la inversión de la matriz $H$, que de lejos es el paso mas costoso en términos computacionales para el algoritmo de minimización. Por tanto, el tamaño de la matriz Hessiana, que en última instancia está definido por el número de partículas que componen el sistema, es clave en la elección del algoritmo de minimización a utilizar.

El método BFGS (Broyden, Fletcher, Goldfarb and Shanno ${ }^{135}$ ), utilizado por defecto en GULP, agrega mejoras sustanciales al método de los gradientes conjugados, sin embargo la selección final de la metodología a utilizar dependerá tanto del tamaño del sistema como de las características de las funciones que describen la superficie de energía potencial.

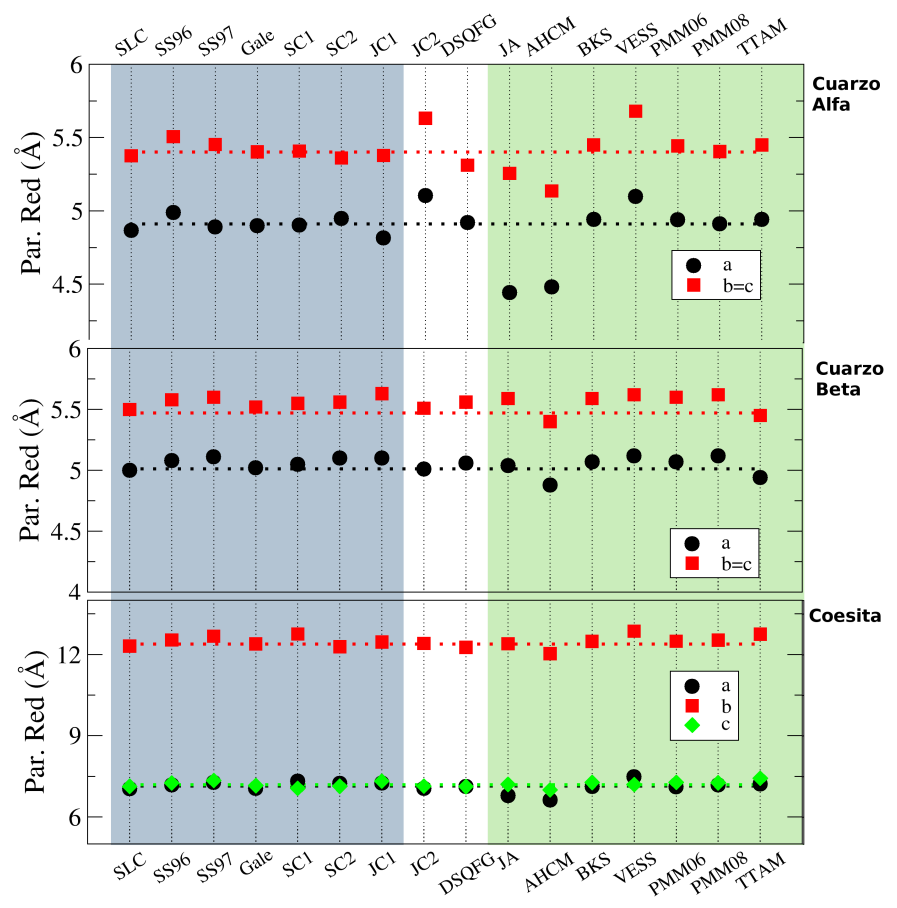

Figura 3.3: Parámetros de red para cuarzo $\alpha$ (arriba), cuarzo $\beta$ (medio) y coesita (abajo) calculados a partir de campos de fuerza interatómicos. Las líneas punteadas hacen referencia a los valores medidos experimentalmente. Laz zonas coloreadas especifican los campos de fuerza con cargas formales y modelo core-shell (azul) y cargas parciales e iones rígidos (verde). En una zona intermedia están los modelos JC2 y DS que representan las interacciones electrostáticas a través de cargas formales en combinación con iones rígidos. En la zona verde se excluye el CF PMM08 que incluye el modelo shell con cargas parciales. 
Las tablas A1-A21 muestran los valores calculados para propiedades estructurales, mecánicas y dieléctricas para polimorfos silíceos y sus correspondientes valores experimentales. Las propiedades estructurales calculadas son los parámetros de red, distancias de enlace $\mathrm{Si}-\mathrm{O}$ y $\mathrm{Si}-\mathrm{Si}$, así como los ángulos entre tripletas $\widehat{\operatorname{SiOS} i}$ y $\widehat{O S i O}$, se incluyen las variaciones de las distancias de enlace, así como los límites superior e inferior de los ángulos de enlace $\widehat{S i O S i}$ y $\widehat{O S i O}$.

La figura 3.3 muestra la comparación entre parámetros de red calculados utilizando campos de fuerza y los valores experimentales para polimorfos silíceos de alta densidad. En la zona correspondiente a los CF que utilizan el modelo del core-shell en conjunto con cargas formales podemos ver en general una mejor reproducción de los valores experimentales correspondientes. Para estos potenciales, las predicciones de las longitudes de enlace $\mathrm{Si}-\mathrm{O}$ se correlacionan con los valores de parámetros de red. Por ejemplo, la sobreestimación de los CF SS96 y SS97 en cuanto a los parámetros de red tiene como razón la sobreestimación de las longitudes de enlace $\mathrm{Si}-\mathrm{O}$, además de la predicción de algunos ángulos $\widehat{\operatorname{SiOS}} i$ con valor de $180^{\circ}$. La magnitud de la distancia media Si-Si tambien registra un valor por encima del valor experimental para los potenciales SS.

\subsection{Campos de Fuerza}

En términos generales la función de energía potencial $(V)$ utilizada en simulaciones atomísticas contiene los siguientes términos:

$$
V=V^{\text {coul }}+V^{\text {core-shell }}+V^{\text {enlazante }}+V^{\text {no-enlazante }}
$$

siendo $V^{\text {coul }}$ la contribución electrostática, $V^{\text {core-shell }}$ el término que define la utilización o no del modelo core-shell para incluir efectos de porlarización, $V^{\text {enlazante }}$ agrupa los términos que controlan las interacciones enlazantes intramoleculares y $V^{\text {no-enlazante }}$ describe las formas funcionales que definen interacciones débiles de atracción y dispersión intra e interatómicas.

\subsubsection{Términos Electrostáticos}

El término $V^{\text {coul }}$ define el aporte energético que resulta de las interacciones electrostáticas entre partículas, por tanto se trata de una sumatoria sobre todas las cargas puntuales $i \neq j$, con forma funcional

$$
V^{\text {Coul. }}=\sum_{i=1}^{N} \sum_{j \neq i \neq 1}^{\infty} \frac{q_{i} q_{j}}{r_{i j}}
$$


donde $r_{i j}$ representa la separación entre las cargas. A pesar de la aparente simplicidad en la definición funcional, este término representa la mayor contribución a la energía potencial del sistema, y su evaluación matemática es sin duda extremadamente desafiante. La dificultad en el cálculo deviene de la convergencia condicional que resulta de la aplicación de condiciones periódicas. Este problema es elegantemente resuelto utilizando el formalismo de Ewald, que divide la interacción Coulómbica en dos series convergentes, una en espacio real y otra en el espacio recíproco o espacio de Fourier. ${ }^{136}$

El uso de condiciones periódicas de frontera hacen que el algoritmo de Ewald presente un gasto computacional asociado a $\mathcal{O}\left(N^{2}\right)$, donde $N$ representa el numero de cargas puntuales en el sistema. Algunos algoritmos alternativos han sido desarrollados para aumentar la eficiencia del modelo de Ewald. Por ejemplo, el método "Particle-Particle Particle-Mesh" es una modificación del modelo original de Ewald, con una carga computacional asociada a $N \ln (N)$, y se basa en el método "Particle-Mesh" donde las interacciones son interpoladas a partir de un "grid" y las fuerzas se integran a partir de este grid resolviendo la ecuación de Poisson de forma discreta, o numérica. ${ }^{137}$

Una forma alternativa para la evaluación del término Coulómbico es presentada por Wolf, ${ }^{138}$ con un método de cálculo que mejora la convergencia de la ecuación 3.5 basada en la observación de que el término electrostático es de corto alcance, y asegurando además el equilibrio electrostática dentro de la esfera de influencia especificada para el sistema. Además, la utilización de una función de "Dampening", convenientemente parametrizada, permite calcular con gan precisión el término de energía electrostático para funciones de tipo Eq. 3.5. Este método es muy económico en gasto computacional y es usado como alternativa al método original de Ewald. ${ }^{97,121-123}$

La segunda parte de la ecuación de energía potencial 3.4 define los términos en los que puede incluirse la polarizabilidad atómica a partir del modelo CoreShell. Como regla general, únicamente los aniones están divididos en Core and Shell, representando de esta manera un par de cargas puntuales de signo contrario, donde la carga positiva está concentrada en el Núcleo (Core) y la negativa en la corteza electrónica (Shell). Estas cargas puntuales están conectadas a traves de un muelle armónico, con constante de fuerza $k$ y distancia entre cargas dada por $r_{c-s}$. La utilización del modelo Core-Shell, tiene el efecto de mejorar el tratamiento electrostático del sistema, mejorando a su vez los resultados en cálculos de propiedades estáticas como constantes dieléctricas y en la obtención de constantes de red mas cercanas a los modelos reales.

$$
V^{\text {core-shell }}=k r_{c-s}^{2}
$$



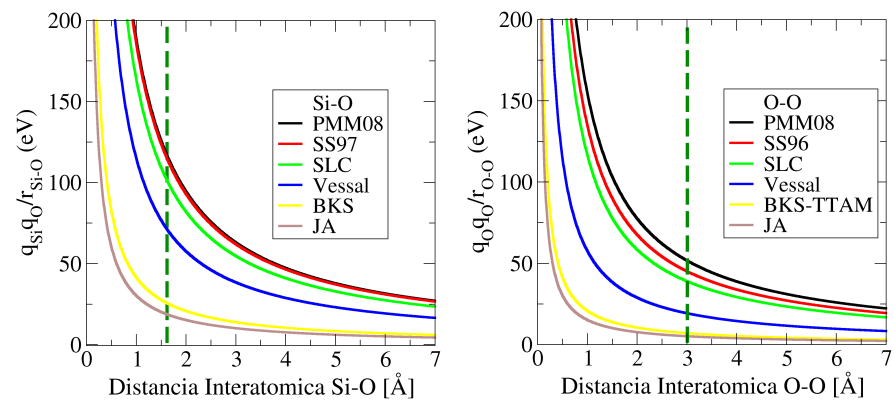

Figura 3.4: Energía de interacción Coulómbica entre átomos de $\mathrm{Si}-\mathrm{O}$ y $\mathrm{O}-\mathrm{O}$ para los campos de fuerza JA, BKS y TTAM (iones rígidos) y PMM08, SS97 y SLC (Core-Shell).

Un ejemplo de la utilización exitosa de este modelo en cálculos de dinámica molecular es el trabajo de Tilocca et al. ${ }^{118}$ La utilización del modelo Core-Shell en sus cálculos sugiere una mejora en la predicción de la estructura tetraédrica del óxido de silicio, debido a la representación mas rigurosa de los enlaces intertetraédricos en la estructura y una mejor descripción del fenómeno de polarización local. De forma general, podemos predecir que la inclusión del modelo CoreShell en simulación de sistemas con moléculas polares adsorbidas representaría una mejora en la descripción de las interacciones intermoleculares, y por tanto se podrían dar mejores predicciones en propiedades que dependieran de la fenomenología intermolecular, tales como energías de adsorción y coeficientes de difusión.

Para la representación de las interacciones electrostáticas en zeolitas y materiales silíceos, la elección de las cargas puntuales es primordial, ya que estas interacciones tienden a ser dominantes en la expresión de energía potencial (ver escalas en la figura 3.5). Para efectos prácticos, la asignación de estados de oxidación formales en combinación con el modelo Core-Shell, normalmente aplicado solo a las partículas aniónicas susceptibles de sufrir polarización, permite el estudio de sistemas inhomogéneos, e.g. sólidos cristalinos con defectos en zonas específicas, donde la asignación de cargas parciales se puede limitar a los fragmentos que componen la zona de interés. Otro caso de interés se da cuando la inhomegeneidad en el sistema se debe a la existencia de moléculas de diferente naturaleza, como es el caso en sistemas zeolita-adsorbato. En este caso las cargas formales se pueden asignar al sólido cristalino y las cargas formales se asocian a los átomos que componen las moléculas adsorbidas.

La asignación de cargas formales sin la inclusión del modelo Core-Shell no es muy recomendable, ya que los términos de interacción electrostática resultan 


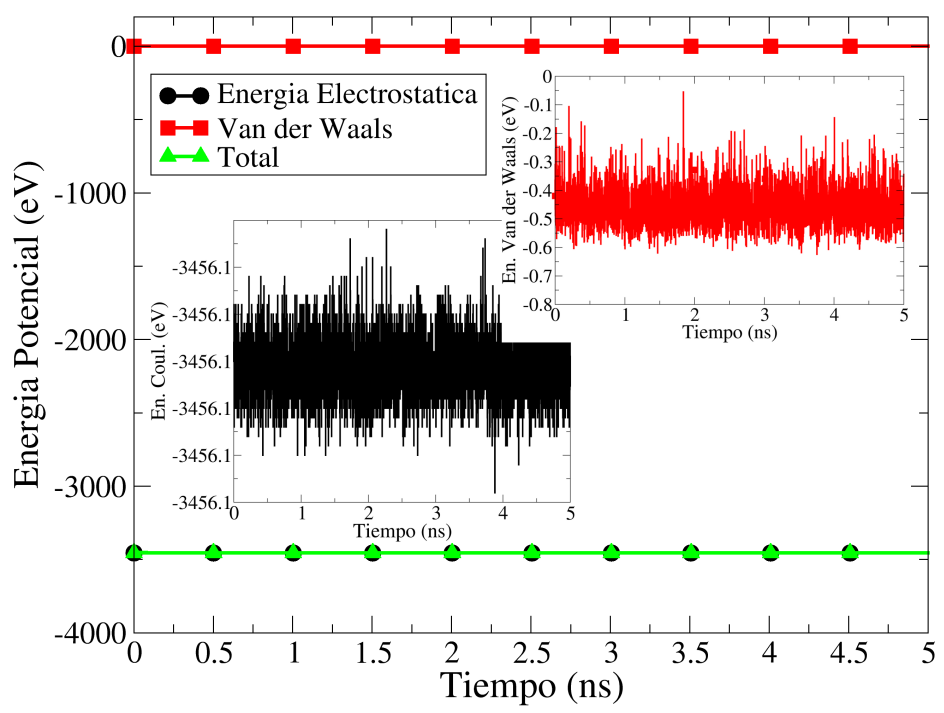

Figura 3.5: Energía potencial total y sus componentes, electrostático y de Van der Waals, para un sistema zeolita-adsorbato (Zeolita LTA y metano) simulado utilizando el método de dinámica molecular en el colectivo canónico (NVT) a $600 \mathrm{~K}$. El campo de fuerza utilizado es el BKS. Las escalas de cada uno de los términos de interacción se resaltan para mostrar la influencia relativa de cada uno en referencia al valor total de la energía potencial.

demasiado cohesivos (en el caso de interacciones atractivas). Este es el caso del modelo de Vessal, que tal como puede apreciarse en la figura 3.4 resulta en valores de interacción electrostática demasiado alta, $E(r=1,62)_{\text {Coul }} \sim 70 \mathrm{eV}$, en comparación con modelos de tipo ión rígido y cargas parciales BKS o JA, los cuales presentan valores de energía electrostática $E(r=1,62)_{\text {Coul }} \sim 20 \mathrm{eV}$. La asignación de las cargas se da generalmente en base a cálculos mecano-cuánticos, teniendo en cuenta que las cargas definidas según la metodología de Mulliken no son la mejor elección, debido a la gran dependencia de las cargas calculadas con respecto al conjunto de funciones que representan la población electrónica del sistema. Cargas calculadas utilizando el modelo del Tensor Effectivo de Cargas de Born ha demostrado que la elección de cargas para modelos de tipo Core-Shell es bastante acertado, ya que reproduce resultados mecano-cuánticos con un alto grado de precision. ${ }^{139} \mathrm{El}$ tensor efectivo de cargas es una cantidad que puede obtenerse a partir del ajuste del potencial a datos dieléctricos experimentales, sin embargo, existe la posibilidad de obtenerlo también a partir de cálculos ab initio. 

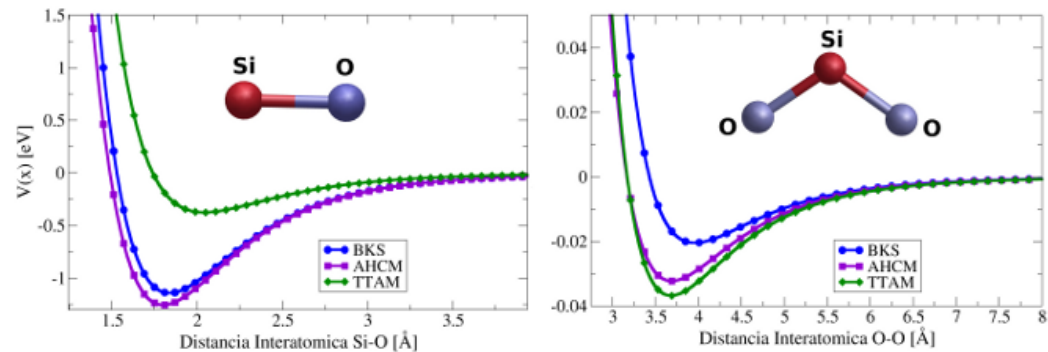

Figura 3.6: Energía de interacción no enlazante entre átomos de $\mathrm{Si}-\mathrm{O}$ y $\mathrm{O}-\mathrm{O}$ para los campos de fuerza AHCM, BKS y TTAM. La energía de interación está descrita por medio de funciones de Buckingham en cada caso, con parámetros específicos para cada uno de los potenciales. Estos tres potenciales tienen en común la descripción de las partículas a través de cargas parciales y iones rígidos.

\subsubsection{Términos Enlazantes}

Tres de los modelos seleccionados (DSQFG, SC2 y SB), utilizan una función armónica entre dos partículas para describir las interacciones $\mathrm{SiO}_{4 / 2}$. Este tipo de función armónica es simplemente un expansión de Taylor de segundo orden en términos de la distancia interatómica $r_{i j}$ entre parejas $\mathrm{T}-\mathrm{O}$, tal como se describe en la ecuación 3.7. La definición de la conectividad intramolecular de esta manera representa una forma sencilla y eficiente computacionalmente para el cálculo de las fuerzas que dominan el sistema, además de permitir la transferencia de energía intermolecular, lo cual supone la posibilidad de llevar a cabo los cálculos dentro del colectivo microcanónico, NVE. Algunos ejemplos de la utilización modelos basados en términos interatómicos enlazantes para la prediccion del comportamiento de adsorbatos en materiales porosos son los trabajos de Hedin et al. ${ }^{140,141}$

$$
V^{\text {Harmonic-Two-Body }}=\frac{k_{i j}}{2}\left(r_{i j}-r_{0}\right)^{2}
$$

La forma funcional armónica entre tres partículas es utilizada para describir explícitamente la hibridación $s p 3$ asociada al carácter covalente del enlace $\mathrm{Si}-\mathrm{O}$. La desviación del ángulo $\theta$ con respecto al ángulo de equilibrio $\left(\theta_{0}=109,47^{0}\right)$ es representada por una constante de fuerza, $k$, la cual representa la rigidez de la tripleta $\mathrm{O}-\mathrm{Si}-\mathrm{O}$. Los modelos SLC, SS96-97, JC1-2 y SC1-2 utilizan este tipo de forma funcional para el estudio de propiedades estructurales y de reactividad en catalizadores zeolíticos. ${ }^{72,95,117,142,143}$ 


\subsubsection{Términos No-Enlazantes}

La mayoría de los campos de fuerza seleccionados, utilizan una descripción de las interacciones $\mathrm{Si}-\mathrm{O}$ basadas en la forma funcional de Buckingham. En caso de los potenciales que describen las partículas aniónicas de la red cristalina por medio del modelo Core-Shell, estas interacciones se dan entre los Shells de los aniones y los cationes. La forma funcional de Buckingham normalmente solo incluye términos para describir las interacciones $\mathrm{Si}-\mathrm{O}$ y $\mathrm{O}-\mathrm{O}$, y se acostumbra excluir las interacciones $\mathrm{Si}-\mathrm{Si}$. La ecuación 3.8 se divide en dos términos: una contribución repulsiva, dada por el término exponencial de repulsión, y una componente atractiva-dispersiva. Esta formulación permite un tratamiento fisicamente razonable de las fuerzas entre los átomos para distancias de interacción de rango medio y bajo. ${ }^{144}$

$$
V^{\text {Buckingham }}=A_{i j} \exp \left(-r_{i j} / \rho_{i j}\right)-\frac{C_{i j}}{r^{6}}
$$

Una variante del potencial de Buckingham es el denominado modelo de cuatroniveles, el cual divide el rango de acción del potencial en regiones de inteacción de acuerdo a:

$$
\begin{aligned}
V^{4-\text { Buckingham }} & =A_{i j} \exp \left(-r_{i j} / \rho_{i j}\right), r_{i j}<r_{1} \\
V^{4-\text { Buckingham }} & =\sum_{m=0}^{5} A_{m} r_{i j}^{m} \rightarrow r_{1}<r_{i j}<r_{2} \\
V^{4-\text { Buckingham }} & =\sum_{m=0}^{3} B_{m} r_{i j}^{m} \rightarrow r_{2}<r_{i j}<r_{3} \\
V^{4-B u c k i n g h a m} & =-\frac{C_{i j}}{r_{i j}^{6}} \rightarrow r_{3}<r_{i j}<r_{c}
\end{aligned}
$$

Esta formulación fue usada por Vessal et al. en el estudio particular de propiedades estructurales de materiales silíceos y de fosfatos vítreos. ${ }^{93}$

Los campos de fuerza de Pedone et al. (PMM06 y PMM08) utilizan una función de tipo Morse para describir las interacciones iónicas de corto alcance. Este tipo de forma funcional es usada normalmente para describir la conectividad en 
sistemas orgánicos covalentes. Tal como se discute en los artículos originales, Pedone et al. solo toman prestada la forma funcional de Morse, que adecuadamente parametrizada es utilizada para la descripción de las interacciones no enlazantes de las unidades de $\mathrm{SiO}_{4 / 2}$. Los parámetros de la ecuación no representan la energía de disociación, asociada con el parámetro $D_{i j}$ o las distancias de equilibrio de enlace $r_{i j}$ en la ecuación 3.13. Estos parámetros deben, por tanto, ser vistos exclusivamente como ajustes a la forma funcional. ${ }^{114}$

$$
V^{\text {Morse }}=D_{i j}\left[\left\{1-\exp \left(-a_{i j}\left(r-r_{0}\right)\right)\right\}^{2}-1\right]
$$

La forma funcional de Urey-Bradley es usada en dos de los potenciales seleccionados: DSQFG y Gale. Esta función es básicamente un potencial de enlace aplicado a una tripleta, donde los átomos terminales de dicha tripleta están conectados a través del mismo átomo central. La ecuación de Urey-Bradley, Eq. 3.14, está definida por la distancia $r_{2-1-3}$, la cual define la distancia entre los átomos de oxígeno terminales de la tripleta $\widehat{O S i O}$, y $r_{2-1-3}^{0}$ representa la distancia de equilibrio. Este modelo se desarrolló para reducir el costo computacional asociado a funciones entre tres partículas ${ }^{96}$ en la minimización de energías libres de silicatos y en el estudio de propiedades dinámicas de zeolitas en cálculos de dinámica molecular. ${ }^{145,146}$

$$
V^{\text {Urey-Bradley }}=\frac{k_{2}}{2}\left(r_{2-1-3}-r_{2-1-3}^{0}\right)^{2}
$$

Finalmente, la función de Vessal entre tres partículas, definida por la ecuación 3.15:

$$
V^{v e s s a l}=\frac{1}{4} A_{i j k} B_{i j k}^{2} \exp \left(-\frac{r_{i j}}{\rho_{1}}\right) \exp \left(-\frac{r_{k j}}{\rho_{2}}\right)
$$

donde

$$
A_{i j k}=\frac{k_{i j k}}{2\left(\theta_{0}-\pi\right)^{2}}
$$

y

$$
B_{i j k}=\left(\theta_{0}-\pi\right)^{2}-\left(\theta_{i j k}-\pi\right)
$$

es usada para representar el ángulo en las tripletas $\widehat{O S i O}$ o $\widehat{S i O S i}$. En los potenciales $\mathrm{AHCM}^{112}$ y JA, ${ }^{113}$ el potencial de Vessal se usa para modelar exclusivamente la interacción angular $\widehat{\mathrm{OSiO}}$, mientras que en los potenciales de Sastre y Corma (SC1-2) ${ }^{116}$ la variación del ángulo $\widehat{O S i O}$ se modela a través de una función armónica y el ángulo $\widehat{\operatorname{SiOS}} i$ está representado por la ecuación de Vessal. Esta forma funcional fué originalmente desarrollada para modelar la estructura amorfa de la sílice, y otros sistemas en los que existe una distribución amplia de ángulos, ya sea $\widehat{O S i O}$ o $\widehat{\operatorname{SiOS}} i .^{93}$ 


\title{
Capítulo 4
}

\section{Difusión en Zeolitas: Influencia de la Flexibilidad de la Red}

\begin{abstract}
En este capítulo se muestra la aplicación de la técnica de dinámica molecular en el estudio de las características estructurales de las zeolitas Si-CHA, Si-SAS y Si-LTA, las cuales corresponden a cristales nanoporosos con canales de diferente dimensionalidad interconectados por ventanas de dimensiones moleculares. La característica común a las estructuras escogidas es la presencia de anillos de 8 unidades tetraédricas de silicio. Inicialmente se estudian las fluctuaciones de la red debidas a efectos térmicos. Con el fin de tomar en cuenta el efecto del potencial interatómico que modela las interacciones en la red de la zeolita, hemos utilizado dos modelos diferentes: el potencial de van Beest, et al. (BKS) y el de Pedone, et al. (PMM).
\end{abstract}

La separación de mezclas olefina/parafina (ejemplo $\mathrm{C}_{2} \mathrm{H}_{6} / \mathrm{C}_{2} \mathrm{H}_{4}, \mathrm{C}_{3} \mathrm{H}_{8} / \mathrm{C}_{3} \mathrm{H}_{6}$ ) es un campo de investigación singularmente activo en la actualidad debido a la creciente demanda de materias primas de alta pureza en la industria y al altísimo grado de consumo energético necesario para llevar a cabo dichas operaciones de separación. ${ }^{147}$ Los procesos de separación basados en zeolitas intercambiadas se llevan a cabo a través de la formación de complejos de tipo $\pi$, los cuales se forman cuando iones de $\mathrm{Cu}$ o Ag, previamente adicionados a la estructura zeolítica, interaccionan con las moléculas insaturadas, ${ }^{148,149}$ mientras que en el caso de separaciones que involucran zeolitas puramente silíceas la separación se da por efectos de tamizado molecular. ${ }^{20,21,141,150}$

En este capítulo se estudia la dinámica de la red cristalina y las consecuencias que el modelamiento de la flexibilidad de dicha red tendrá sobre la movilidad de las especies adsorbidas. Para este fin usaremos la técnica de Dinámica Molecular 
(DM), ya que este método proporciona información a nivel atomístico, relevante en las escalas de tiempo y tamaño adecuadas para el estudio de la movilidad de adsorbatos en zeolitas puramente silíceas con ventanas de 8 unidades.

Usualmente los estudios computacionales de especies adsorbidas en zeolitas han considerado de menor importancia la inclusión de grados de libertad vibracionales de la red silícea, ${ }^{151}$ sin embargo en situaciones de gran confinamiento el factor de flexibilidad es de esperar que tenga un alto grado de relevancia. ${ }^{81,152,153}$ En esta línea de ideas, el trabajo de van der Berg et al. ${ }^{154,155}$ acerca de la influencia de la flexibilidad de la red en la difusión de hidrógeno molecular en zeolitas con anillos de 6 unidades tetraédricas, muestra el alto grado de dependencia de la dinámica interna del adsorbato respecto a la flexibilidad de la red. Este efecto se hace aún mas evidente cuando se analiza el efecto de los cationes en zeolitas intercambiadas, ya que dichos cationes tienen la capacidad de afectar el nivel de flexibilidad de la red, y por tanto, tienen la capacidad de afectar directamente la difusión del adsorbato.

El ansazt de nuestro estudio se basa en la identificación de las ventanas, o anillos de 8 unidades tetraédricas, como los cuellos de botella para la evolución difusional de los adsorbatos, por tanto un conocimiento atomístico detallado de la dinámica de dichas ventanas y su influencia en el grado de movilidad inter-poro es de gran importancia. En el caso específico de propano y propileno como adsorbatos, el tamaño de la ventana se convierte en un factor crítico, ya que el diámetro cinético de las moléculas es casi identico al diámetro crítico de la ventana.

Tal como se vió en el capítulo anterior, el factor más importante a tener en cuenta para la representación adecuada de las interacciones intramoleculares del cristal es el potencial interatómico. En este trabajo, la influencia del potencial se analizó comparando los resultados obtenidos a partir de dos potenciales: el potencial de van Beest, Kramer y van Santen (BKS) ${ }^{104}$ y el Pedone, Malavasi, Menziani, Cormack y Segre (PMM), ambos potenciales con modelo de ión rígido y cargas parciales. ${ }^{114}$

El potencial BKS ha sido escogido por su capacidad para modelar y predecir la estructura de diferentes zeolitas con un alto grado de precisión, aunque sus limitaciones para la representación de la densidad de estados vibracionales está muy bien documentada. ${ }^{101} \mathrm{El}$ potencial PMM, a diferencia del modelo BKS, utiliza la forma funcional de Morse para la descripción de las interacciones no enlazantes de corta distancia. Este potencial ha sido parametrizado a partir de información estructural de una serie de óxidos silíceos.

La diferente representación de las interacciones no enlazantes de van der Waals en los potenciales (Buckingham y Morse asociadas al BKS y PMM repectivamente) servirá para la identificación de las diferencias estructurales, dinámicas y en la movilidad de los adsorbatos que se generan al utilizar diferentes modelos 

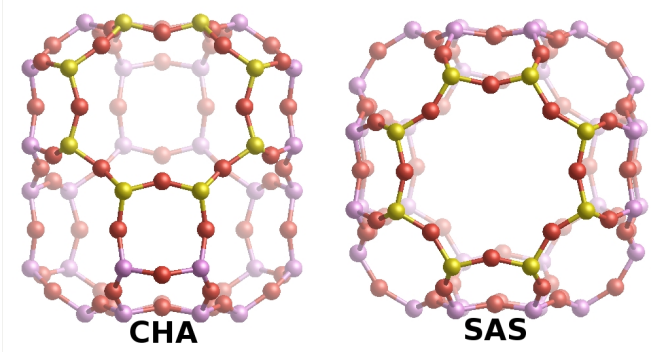

Figura 4.1: Esructura de las zeolitas utilizadas

para la representación de la estructura cristalina. Como nota final, la selección de estos dos potenciales para la representación de la red se da también en base a su facilidad de implementación computacional.

En las secciones siguientes se describen las estructuras utilizadas, (sección 4.1), se dan los detalles particulares del método de simulación especificando los detalles de los potenciales seleccionados, los algoritmos usados para el análisis de la información obtenida de las simulaciones (sección 4.2), se pesentan y discuten los resultados obtenidos (sección 4.3) y finalmente las conclusiones y comentarios finales se presentan en la sección 4.4.

\subsection{Modelos}

Se han seleccionado dos estructuras compuestas enteramente por unidades silíceas de tipo SAS y CHA. La red zeolitica de tipo SAS, o zeolita SSZ-73, es una estructura con canales unidimensionales formados por anillos compuestos por 4,6 y 8 unidades silíceas tetraédricas, con grupo espacial $I 4 / \mathrm{mmm} .{ }^{10} \mathrm{La}$ zeolita SSZ-73 es inusual porque si bien posee canales unidimensionales formados por poros relativamente pequeños también posee un un volumen de microporo muy grande, debido a las dimensiones de su celda. SSZ-73 tiene un volumen de microporo de $0,25 \mathrm{~cm}^{3} / \mathrm{g}$, el cuál es sorprendentemente alto para un tamiz molecular unidimensional. Otro factor inesperadamente sobresaliente es su gran área superficial de cerca de $585 \mathrm{~m}^{2} / \mathrm{g}$.

La zeolita de tipo CHA posee una celda unitaria romboédrica formada por 36 atoms, con simetría $R 3 m$ y con cuatro ángulos Si-O-Si cristalográficamente diferentes. ${ }^{128}$ Está formada igualmente por una serie de anillos de 4, 6 y 8 unidades siíceas tetraédricas en un arreglo 3-dimensional de celdas interconectadas (Ver figura 4.1). Los detalles estructurales de las estructuras seleccionadas se detallan en la tabla 4.1. 


\begin{tabular}{lccccccc}
\hline Zeolitea & a $[\AA]$ & $\mathbf{b}[\AA]$ & $\mathbf{c}[\AA]$ & $\alpha[\mathbf{G r a d}]$ & $\beta[\mathbf{G r a d}]$ & $\gamma[\mathbf{G r a d}]$ & Vol $\left[\AA^{3}\right]$ \\
\hline SSS-73 $^{\mathbf{1 0}}$ & 14.104 & 14.104 & 10.187 & 90.00 & 90.00 & 90.00 & 2026.50 \\
Si-CHA $^{\mathbf{1 2 8}}$ & 13.675 & 13.675 & 14.767 & 90.00 & 90.00 & 120.00 & 2391.54
\end{tabular}

Tabla 4.1: Zeolitas Si-CHA y Si-SAS: parámetros de red y volúmenes de celda

\subsection{Métodos}

\subsubsection{Detalles de Cálculo}

El método de dinámica molecular, descrito a fondo en el Capítulo 3, se utiliza para simular la dinámica de la red y la difusión de adsorbatos adsorbidos en dichas estructuras cristalinas, utilizando para ello el código DL_POLY. ${ }^{156}$ Además de la evaluación de la influencia del potencial se ha estudiado la forma en que la temperatura afecta la estructura y por consiguiente la dinámica de los adsorbatos. ${ }^{157}$ Las dimensiones de la celda de simulación se obtuvieron a partir del escalamiento de la celda unidad, utilizando un factor de 2 para cada parámetro de red.

Las simulaciones se llevaron a cabo utilizando el algoritmo "Velocity Verlet" para la integración de las ecuaciones de movimiento de Newton, con tiempo de integración de 1 fs en todos los casos. La carga de adsorbato se fijó en 4 moléculas por celda de simulación, carga lo suficientemente baja para minimizar la influencia que las interacciones adsorbato-adsorbato tendrían sobre la dinámica del sistema, ya que el objetivo es poner de relieve el efecto de la topología, cuya mayor influencia es visible a través de las interacciones red-adsorbato.

Los sistemas simulados se evaluaron dentro de los colectivos canónico (NVT) y microcanónico (NVE). El colectivo NVT se utiliza para el caso de la red fija, mientras que el colectivo NVE se usa en condiciones de red flexible, tomando ventaja del hecho que los grados de libertad vibracionales del cristal actúan efectivamente como baño térmico. Las velocidades de las partículas móviles del sistema se inicializaron a partir de distribuciones de Maxwell-Boltzmann, con las cuales se obtienen trayectorias de pre-equilibración, con reemplazo de las velocidades de las partículas cada 10 fs por un tiempo de simulación de 10 ps. Seguidamente, un período de equilibrio de 200 ps es usado para relajar el sistema hasta que la variación de energía y temperatura se encuentran dentro de límites estadisticamente confiables. Este procedimiento es especialmente crítico al llevar a cabo la simulación del proceso de obtención de parámetros de red a partir de variaciones térmicas. Para este caso específico, se usó el barostato/termostato de Berendsen para el control de presión y temperatura.

Una vez finalizado el proceso de equilibración del volumen, las configuracio- 
nes obtenidas se usan como puntos iniciales para trayectorias dentro del colectivo NVE. Las simulaciones se inician con períodos de equilibración de 20 ps, seguidas de períodos de producción de hasta $15 \mathrm{~ns}$. El análisis de las trayectorias se llevó a cabo a partir de los archivos generados por DL_POLY, utilizando como herramienta principal funciones de distribución de pares de partículas, las cuales se obtienen a partir de la relación:

$$
G(r)_{a b}=\frac{1}{G_{a b}}\left[\frac{d N_{a b}}{d V}\right]
$$

donde $N_{a b}$ es el número promedio de parejas entre partículas a y b separadas por una distancia comprendida entre $r$ y $r+d r, G_{a b}$ es la densidad de partículas a y b, y $\mathrm{V}$ es el volumen del sistema.

\subsubsection{Potencial Interatómico}

La superficie de energía potencial total del sistema simulado involucra tres términos: el potencial de la red, $V^{Z e o}$, el potencial del adsorbato, $V^{A d s}$, y las interacciones intermoleculares red-adsorbato, $V^{\text {Zeo-Guest/Guest-Guest }} \mathrm{y}$ adsorbatoadsorbato.

$$
V^{T o t a l}=V^{Z e o}+V^{A d s}+V^{Z e o-A d s / A d s-A d s}
$$

\section{Potencial para la Red Cristalina}

La función descriptora del potencial intramolecular cristalino se representó en la forma:

$$
V^{Z e o}=V^{Z e o-S h o r t}+V^{C o u l}
$$

Con un término Coulombico que describe las interacciones electrostáticas de largo alcance, y un término de corta influencia para modelar la energía dispersiva/repulsiva entre pares de partículas. La evaluación del término electrostático se llevó a cabo usando el formalismo Particle Mesh Ewald, ${ }^{136}$ incluído dentro del código DL_POLY. Para la evalución de los términos de corto alcance hemos seleccionado dos formas de potenciales diferentes. El primero coresponde al modelo de Born-Mayer para sólidos iónicos parametrizado por van Beest et al. (BKS), ${ }^{104}$ y el segundo es el potencial desarrollado por Pedone et al. ${ }^{114}$ Estos potenciales fueron descritos en profundidad en el capítulo anterior, sin embargo cabe resaltar las diferencias más sobresalientes entre ellos.

El ptencial BKS, aplicado a los pares oxígeno-oxígeno y oxígeno-silicio (Ver Eq. 4.4), se divide en dos términos, el primero representa la contribución repulsiva 


\begin{tabular}{|c|c|}
\hline$B K S$ & PMM \\
\hline$S i[\mathbf{e}]+2.4$ & $S i[\mathbf{e}]+2.4$ \\
\hline$O$ [e] -1.2 & $O$ [e] -1.2 \\
\hline Buckingham (Eq 4.4) & Morse (Eq. 4.5) \\
\hline$A_{S i-O}[\mathrm{eV}]=18003.7572$ & $D_{e}[\mathrm{ev}]=0.340554$ \\
\hline$A_{O-O}[\mathrm{eV}]=1388.7730$ & $a_{s i-O}\left[\AA^{-2}\right]=2.006700$ \\
\hline$\rho_{S i-O}[\AA \AA]=0.205205$ & $r_{S i-0}[\AA ̊]=2.100000$ \\
\hline$\rho_{O-O}[\AA]=0.362319$ & $D_{e}[\mathrm{eV}]=0.042395$ \\
\hline$C_{S i-O}\left[\right.$ evÅ $\left.^{6}\right]=133.5381$ & $a_{O-O}\left[\AA^{-2}\right]=1.379316$ \\
\hline$C_{O-O}\left[\mathrm{evA}^{6}\right]=175.0000$ & $r_{O-O}[\AA \AA]=3.618701$ \\
\hline
\end{tabular}

Tabla 4.2: Parámetros para los potenciales BKS y PMM

(término exponencial) y el segundo la contribución dispersiva (término descrito por variación inversa respecto a la separación, con potencia 6):

$$
V_{B K S}^{Z e o-S h o r t}=A_{i j} \exp \left(\frac{-r_{i j}}{\rho_{i j}}\right)-\frac{C_{i j}}{r^{6}}
$$

El modelo PMM utiliza una función de tipo morse para la descripción de las interacciones interpartículas, entendiendo los diferentes parámetros de la ecuación como simples parámetros de ajuste, sin ningún significado relevante desde el punto de vista químico. ${ }^{114}$

$$
V_{P M M}^{Z e o-S h o r t}=D_{i j}\left\{\left[1-\exp \left(a_{i j}\left(r-r_{0}\right)\right)\right]^{2}-1\right\}
$$

Los parámetros de los potenciales se describen en la tabla 4.2.

\section{Potencial para los Adsorbatos}

La función de energía potencial de los adsorbatos se tomó de los modelos descritos por Oie et al. ${ }^{158}$ En forma general, el potencial de los adsorbatos puede describirse a partir de términos de interacción entre pares de partículas enlazantes $V^{2 B}$, términos angulares actuando entre tripletas $V^{3 B}$ y términos que especifican las variaciones entre ángulos dihedros que se forman entre cuatro partículas $V^{\text {Torsion }}$, además de las interacciones coulómbicas entre cargas puntuales $V^{\text {Coul }}$ :

$$
\begin{gathered}
V^{\text {Guest }}=V^{2 B}+V^{3 B}+V^{\text {Torsion }}+V^{\text {Coul }} \\
V^{2 B}=\frac{1}{2} k_{2 B}\left(r_{i j}-r_{i j}^{0}\right)^{2}
\end{gathered}
$$




$$
\begin{gathered}
V^{3 B}=\frac{1}{2} k_{3 B}\left(\theta_{i j k}-\theta_{i j k}^{0}\right)^{2} \\
V^{\text {Torsion }}=k_{4 B}\left[1+\cos \left(n \phi_{i j k}-\phi_{i j k}^{o}\right)\right]
\end{gathered}
$$

los parámetros para los diferentes potenciales se dan en la referencia original.

Finalmente, las interacciones entre partículas que pertenencen a moléculas diferentes (adsorbato-adsorbato y adsorbato-zeolita) se describen a través de potenciales de tipo Lennard-Jones de acuerdo a la ecuación:

$$
V^{\text {Zeo-Guest } / \text { Guest-Guest }}=4 \varepsilon_{i j}\left[\left(\frac{\sigma_{i j}}{r_{i j}}\right)^{12}-\left(\frac{\sigma_{i j}}{r_{i j}}\right)^{6}\right]+V^{\text {Coul }}
$$

donde $\varepsilon_{i j}$ representa la profundidad del pozo de potencial, $\sigma_{i j}$ describe el diámetro cinético de cada partícula y $r_{i j}$ referencia la distancia entre las partículas $i$ y $j$, respectivamente.

\subsection{Resultados y Discusión}

La evaluación inicial de los potenciales interatómicos se llevó a cabo calculando parámetros de red, distancias de enlace y ángulos entre tripletas enlazadas en las estructuras cristalinas Si-SAS y Si-CHA. Estos cálculos se llevaron a cabo por medio de la técnica de optimización estructural a partir de la minimización de la energía, utilizando el algoritmo BFGS (Broyden-Fletcher-GoldfarbShanno ${ }^{135}$ ) disponible en GULP. ${ }^{132,133}$

La tabla 4.3 muestra una relación de las propiedades estructurales calculadas y los valores experimentales existentes. Para las dos estructuras se obtiene una predicción consistente por parte de los dos potenciales, arrojando parámetros de red $(\mathrm{a}, \mathrm{b})$ y distancias de enlace $(\mathrm{Si}-\mathrm{O})$ por encima de los valores experimentales para los cálculos con el potencial BKS y menores en el caso del PMM, con desviaciones tambien consistentemente mayores para el modelo PMM. En el caso de los ángulos entre tripletas $(\mathrm{Si}-\mathrm{O}-\mathrm{Si})$ las desviaciones en los dos casos son positivas, con mejores predicciones obtenidas a partir del modelo BKS.

\subsubsection{Variación Estructural con la Temperatura}

Como segunda prueba del funcionamiento de los potenciales se ha estudiado la influencia de la temperatura en la descripción de los parámetros de la red. Para este efecto se escogió la estructura Si-CHA como estructura de prueba inicial, 


\begin{tabular}{|c|c|c|c|c|c|c|}
\hline Potencial & $\mathbf{a}[\AA ̊]$ & c [̊̊] & $S i-O[\AA ̊]$ & $S i-S i[\AA ̊]$ & $\angle S i-O-S i$ & $\angle O-S i-O$ \\
\hline \multicolumn{7}{|c|}{ SAS } \\
\hline \multirow[t]{2}{*}{ BKS } & 14.43 & 10.28 & $1.594-1.616$ & $3.149-3.161$ & $156.154-161.993$ & $106.080-114.073$ \\
\hline & & & 1.608 & 3.152 & 157.454 & 109.396 \\
\hline \multirow[t]{2}{*}{ PMM06 } & 13.564 & 9.668 & $1.497-1.501$ & $2.956-2.982$ & $160.169-167.940$ & $103.197-119.055$ \\
\hline & & & 1.499 & 2.962 & 162.716 & 109.381 \\
\hline $\operatorname{Exp}^{10}$ & 14.1039 & 10.1875 & 1.595 & & 150.1 & \\
\hline \multicolumn{7}{|c|}{ CHA } \\
\hline \multirow[t]{2}{*}{ BKS } & 13.85 & 14.82 & $1.599-1.615$ & $3.121-3.158$ & $153.650-155.701$ & $106.908-114.458$ \\
\hline & & & 1.609 & 3.140 & 154.852 & 109.391 \\
\hline \multirow[t]{2}{*}{ PMM06 } & 12.98 & 14.09 & $1.498-1.499$ & $2.939-2.958$ & $157.3742-162.089$ & $102.383-117.969$ \\
\hline & & & 1.499 & 2.953 & 160.440 & 109.361 \\
\hline $\operatorname{Exp}^{128}$ & 13.53 & 14.75 & 1.603 & & $148.4 / 148.0$ & 109.47 \\
\hline
\end{tabular}

Tabla 4.3: Comparativa entre parametros de red experimentales y calculados para las zeolitas Si-CHA y Si-SAS.

debido a la existencia de mediciones experimentales bajo condiciones de temperatura variable. Los detalles de los cálculo y resultados se presentan en la tabla 4.4. El volumen de la celda unidad reportado por Hedin et al. ${ }^{140}$ es de $2348 \AA^{3}$ a $298 \mathrm{~K}$ y $101,3 \mathrm{kPa}$, y a condiciones similares Woodcock et al. ${ }^{157}$ reportan un valor de $2334 \AA^{3}$. A partir de nuestros cálculos a $300 \mathrm{~K}$ utilizando el potencial BKS, se obtuvo un valor de $2442 \AA^{3}$, el cual se desvía $5 \%$ de los datos experimentales. El potencial PMM da como resultado volúmenes de celda ligeramente mayores que los del BKS. Los resultados a diferentes temperaturas se presentan en la Figura 4.2, en contraste con los resultados de Woodcock et al. ${ }^{157}$

Experimentalmente se encuentra una variación no lineal del volumen de la celda con respecto a la temperatura, tal como se aprecia en la Figura 4.2. Esta variación es parcialmente reproducida por los dos potenciales, aunque se observa una mejor descripción de la variación negativa del volumen respecto a la temperatura con el potencial BKS. El potencial BKS calcula volúmenes que se desvían 4-5\% de los valores experimentales en el rango de temperaturas estudiado, con desviaciones aumentando con el aumento de la temperatura, mientras que el potencial PMM se devía en un porcentaje un poco más alto, entre 6-7\%. Sin embargo, los datos experimentales muestran una variación que se acentúa a temperaturas altas, comportamiento que ninguno de los dos potenciales es realmente capaz de capturar correctamente.

Los datos estructurales para la zeolita Si-SAS se detallan en la tabla 4.5. El volumen experimental medido para la zeolita SSZ-73 a condiciones normales es 


\begin{tabular}{|c|c|c|c|c|}
\hline \multicolumn{5}{|c|}{ Si-CHA } \\
\hline BKS & $D_{1}[\AA \mathbf{\AA}]$ & $D_{2}[\AA]$ & $\langle V\rangle^{N P T}\left[\AA^{3}\right]$ & $\Delta V^{|E x p-C a l c|}[\%]$ \\
\hline $300 \mathrm{~K}$ & $3,83 \pm 0,14$ & $4,20 \pm 0,17$ & $2442 \pm 2,4$ & 4,6 \\
\hline $400 \mathrm{~K}$ & $3,84 \pm 0,16$ & $4,18 \pm 0,19$ & $2439 \pm 2,8$ & 4,6 \\
\hline $600 \mathrm{~K}$ & $3,82 \pm 0,20$ & $4,17 \pm 0,23$ & $2434 \pm 3,6$ & 4,8 \\
\hline $800 \mathrm{~K}$ & $3,82 \pm 0,23$ & $4,16 \pm 0,27$ & $2428 \pm 4,9$ & 5,2 \\
\hline PMM & $D_{1}[\AA]$ & $D_{2}[\AA]$ & $\langle V\rangle^{N P T}\left[\AA^{3}\right]$ & $\Delta V^{|E x p-C a l c|}[\%]$ \\
\hline $300 \mathrm{~K}$ & $3,96 \pm 0,14$ & $4,12 \pm 0,16$ & $2470 \pm 2,0$ & 5,8 \\
\hline $400 \mathrm{~K}$ & $3,95 \pm 0,16$ & $4,11 \pm 0,19$ & $2469 \pm 2,6$ & 5,9 \\
\hline $600 \mathrm{~K}$ & $3,92 \pm 0,19$ & $4,12 \pm 0,23$ & $2467 \pm 3,3$ & 6,2 \\
\hline $800 \mathrm{~K}$ & $3,92 \pm 0,22$ & $4,10 \pm 0,26$ & $2465 \pm 4,3$ & 6,8 \\
\hline EXP & $D_{1}[\AA]$ & $D_{2}[\AA]$ & $V^{E x p}\left[\AA^{3}\right]$ & REF \\
\hline $293 \mathrm{~K}$ & - & - & 2334 & 157 \\
\hline $298 \mathrm{~K}$ & 3,70 & 4,17 & 2348 & 140 \\
\hline $300 \mathrm{~K}$ & - & - & 2392 & 128 \\
\hline
\end{tabular}

Tabla 4.4: Variaciones de las dimensiones de las ventanas y volúmenes de celda, y su dependencia con la temperatura para la zeolita Si-CHA. Las distancias efectivas oxígeno-oxígeno en esta estructura se obtuvieron a partir de trayectorias de DM en el colectivo NVE a las temperaturas especificadas (con configuraciones iniciales obtenidas del cálculo en el colectivo NPT) usando los potenciales BKS y PMM. Las distancias efectivas se presentan como resultado de la sustracción de dos veces el radio de van der Waals correspondiente al oxígeno $(2 \times 1.35=2.7 \AA$, tal como sugiere la International Zeolite Association $\left.{ }^{159}\right)$. Los volúmenes calculados representados por $\langle V\rangle^{N P T}$, hacen referencia al colectivo bajo el que se hace el muestreo, NPT en este caso, y a la desviación estandar correspondiente a la trayectoria. El término de diferencia entre volumen experimental y calculado $\Delta V^{|E x p-C a l c|}$ representa el valor absoluto de dicha diferencia a la temperatura correspondiente. $^{157}$ 


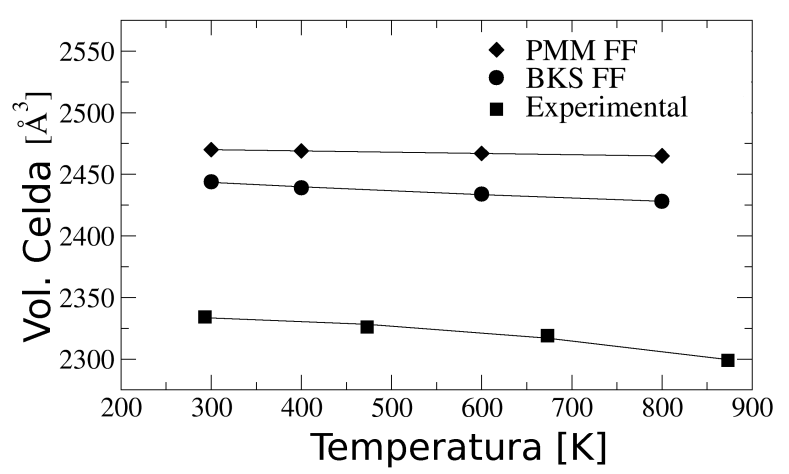

Figura 4.2: Cambio en el volumen de la celda calculado con los potenciales BKS y PMM para $\mathrm{Si}-\mathrm{CHA}$ en el rango de temperaturas entre 300-800 K. Valores experimentales de Woodcock et al. ${ }^{157}$

de $2026,5 \AA^{3} .{ }^{10}$ Nuestros cálculos producen valores de $2119 \AA^{3}$ y $2140 \AA^{3}$ a $300 K$ para los potenciales BKS y PMM, respectivamente, dentro de un intervalo de desviación porcentual de $5-6 \%$ respecto al valor experimental. ${ }^{10}$ Los datos obtenidos sugieren un comportamiento consistente de los potenciales, con valores sobreestimados para los dos potenciales, y una variación ligeramente más acelerada del potencial BKS a altas temperaturas. Hasta la fecha no existen mediciones experimentales para la zeolita SSZ-73, con lo cual no es posible confirmar la precisión de los datos calculados. Sin embargo, la mejor representación obtenida utilizando el potencial BKS para la estructura Si-CHA nos permite tener un mayor grado de confianza sobre la transferibilidad de este potencial a otras estructuras de tipo similar en comparación con el potencial PMM.

\subsubsection{Dinámica de las Ventanas}

Las funciones de distribución de distancia entre pares de átomos de oxígeno, las cuales definen las dimensiones críticas de la ventana, se representan en la figura 4.3. Los datos se han tomado de trayectorias de DM obtenidas en condiciones NVE a diferentes temperaturas, para cada uno de los modelos de energía potencial estudiados.

Las distancias $\mathrm{O}-\mathrm{O}$ efectivas calculadas a $300 \mathrm{~K}\left(D_{1}=3,83 \AA, D_{2}=4,20\right.$ $\AA$ ) para $\mathrm{Si}-\mathrm{CHA}$ con el potencial BKS son ligeramente mayores a las reportadas a similares condiciones por Hedin et al. $\left(D_{1}=3,70 \AA\right.$ y $\left.D_{1}=4,17 \AA\right) .{ }^{140} \mathrm{La}$ diferencia en dimensiones se debe a la sobreestimación de los parámetros de red, 


\begin{tabular}{|c|c|c|c|c|}
\hline \multicolumn{5}{|c|}{ Si-SAS } \\
\hline BKS & $D_{1}[\AA]$ & $D_{2}[\AA]$ & $\langle V\rangle^{\operatorname{Calc}}\left[\AA^{3}\right]$ & $\Delta V^{|E x p-C a l c|}[\%]$ \\
\hline $300 \mathrm{~K}$ & $4,24 \pm 0,16$ & $4,26 \pm 0,16$ & $2119 \pm 2,7$ & 4,6 \\
\hline $600 \mathrm{~K}$ & $4,22 \pm 0,22$ & $4,28 \pm 0,22$ & $2109 \pm 4,3$ & N/A \\
\hline $800 \mathrm{~K}$ & $4,21 \pm 0,25$ & $4,24 \pm 0,25$ & $2102 \pm 5,0$ & N/A \\
\hline PMM & $D_{1}[\AA]$ & $D_{2}[\AA]$ & $\langle V\rangle^{\operatorname{Calc}}\left[\AA^{3}\right]$ & $\Delta V^{|E x p-C a l c|}[\%]$ \\
\hline $300 \mathrm{~K}$ & $4,24 \pm 0,16$ & $4,28 \pm 0,15$ & $2140 \pm 2,3$ & 5.6 \\
\hline $600 \mathrm{~K}$ & $4,25 \pm 0,20$ & $4,26 \pm 0,21$ & $2135 \pm 4,0$ & N/A \\
\hline $800 \mathrm{~K}$ & $4,20 \pm 0,23$ & $4,29 \pm 0,24$ & $2132 \pm 4,9$ & N/A \\
\hline EXP & $D_{1}[\AA]$ & $D_{2}[\AA]$ & $V^{E x p}\left[\AA^{3}\right]$ & REF \\
\hline $298 \mathrm{~K}$ & 4,2 & 4,2 & 2027 & 10 \\
\hline
\end{tabular}

Tabla 4.5: Variaciones de las dimensiones de las ventanas, volúmenes de celda, y su dependencia con la temperatura para la zeolita Si-SAS. Las forma de cálculo de las distancias efectivas oxígenooxígeno, los volúmenes y la diferencia entre volumen experimental y calculado $\Delta V^{|E x p-C a l c|}$ es igual al especificado en el encabezamiento de la tabla anterior, con la salvedad de que los datos experimentales en este caso son tomados de Wragg et al. ${ }^{10}$

y por consiguiente al valor mayor de volumen, obtenidos utilizando el modelo BKS.

La estimación de longitudes de enlace $\mathrm{Si}-\mathrm{O}$ mayores a las experimentales tiene como origen posible una asignación baja de las cargas iónicas asignadas a los átomos de la red, $+2,4$ y $-1,2$ para silicio y oxígeno respectivamente. Estas cargas, las cuales son probablemente bajas, generan fuerzas de repulsión entre partículas del mismo tipo y de atracción entre cargas opuestas posiblemente de menor magnitud a las presentes en el material "real", con lo cual se requiere de términos no enlazantes de atracción y repulsión de mayor magnitud para contrarrestarlas.

Este efecto de compensación es evidente si se comparan los parámetros del modelo BKS con otros potenciales. Evidencia de este hecho lo tenemos en la magnitud del término $C_{6}$ del potencial, parámetro netamente dispersivo, el cual es extremadamente alto $\left(C_{6}=175 \mathrm{eV}\right)$ si se compara con modelos de tipo ion flexible y carga formal, por ejemplo $C_{6}=52,12 \mathrm{eV}$, para el modelo Vessal y aún menor, $C_{6}=27,88 \mathrm{eV}$, para el JC2.

Los puntos máximos en las funciones de distribución representadas en la figura 4.3(a) muestra una disminución progresiva de las distancias $\mathrm{O}-\mathrm{O}$ con aumento de la temperatura, lo cual no es sorprendente dado el caracter negativo del factor de expansión térmica, definido por la relación $\alpha_{v}=\frac{1}{V}\left(\frac{\partial V}{\partial T}\right)_{p}$.

Es de destacar que la disminución de las dimensiones de la ventana no es 

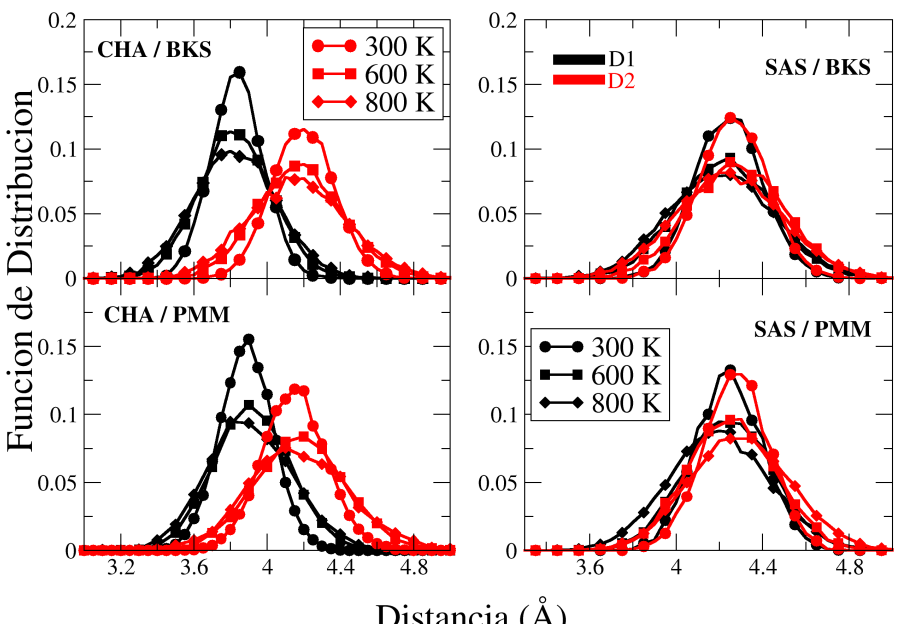

Distancia $(\AA)$

Figura 4.3: Funciones de distribución entre parejas $O-O$, definiendo las distancias como $D_{1}$ y $D_{2}$, en Si-CHA y Si-SAS a partir de trayectorias de DM a 300, 600 y $800 \mathrm{~K}$ usando los potenciales $B K S$ y $P M M$.

simétrica respecto a las dimensiones críticas especificadas, produciéndose una disminución mayor en magnitud en la distancia $D_{2}$. El efecto de esta variación asimétrica en las dimensiones de la ventana tiene como consecuencia estructural la formación de una ventana de forma menos elongada.

Otra consecuencia importante del efecto térmico es el aumento en la magnitud de las fluctuaciones de $D_{1}$ y $D_{2}$. De esta forma, el efecto que tendrá una mayor apertura de las ventanas con respecto a la temperatura será aditivo a la simple excitación térmica del adsorbato. Esta suposición será discutida en el siguiente capítulo, donde se presentarán los resultados de difusión de adsorbatos en estructuras zeolíticas.

La figura 4.3 presenta los valores calculados de la distribución de distancias $\mathrm{O}-\mathrm{O}$ utilizando el potencial PMM y estructura Si-CHA. Las distancias O-O obtenidas a $300 \mathrm{~K}, D_{1}=3,96 \AA$ y $D_{2}=4,12 \AA$, se desvían en mayor medida de los datos experimentales comparado con el modelo BKS para la misma estructura. La distancia $D_{1}$ es mayor y la $D_{2}$ es ligeramente menor en comparación. En consonancia con el comportamiento de la estructura modelada con el potencial BKS, también se obtiene una variación de las dimensiones de las ventanas aumentando con los cambios térmicos. Las dimensiones ligeramente mayores claculadas para este potencial, podrían traducirse en una menor barrera al movimiento difusional 
de adsorbatos en esta estructura.

Del mismo modo, las variaciones en dimensión de las ventanas se han calculado a partir de funciones de distribució de distancias entre pares para la estructura Si-SAS en el mismo rango de temperaturas. Idénticas condiciones de colectivo de muestreo (NVE) y potenciales son utilizados para la zeolita Si-SAS. Los resultados se presentan en la figura 4.3.

Las distancias efectivas $\mathrm{O}-\mathrm{O}$ calculadas con el potencial BKS a $300 \mathrm{~K}\left(D_{1}=\right.$ $4,24 \AA, D_{2}=4,26 \AA$ ) son comparables a las obtenidas con el potencial PMM en iguales condiciones $\left(D_{1}=4,24 \AA\right.$ y $\left.D_{2}=4,28 \AA\right)$, con variación de las dimensiones promedio de la ventana independientes de la temperatura. Los valores experimentales $D_{1}$ y $D_{2}$ reportados para Si-SAS son $D_{1}=D_{2}=4,25$ $\AA$. No existen diferencias apreciables en cuanto a la precisión de los resultados en función del potencial utilizado para esta estructura. Una tendencia similar a la encontrada para la estructura Si-CHA se obtiene para Si-SAS, esto es, valores ligeramente mayores de volumen, y por consiguiente de los parámetros de la red. Los coeficientes de expansión térmica para esta estrucutra concuerdan con los resultados obtenidos para $\mathrm{Si}-\mathrm{CHA}$, aunque en este caso los resultados son puramente predictivos, al no existir información experimental para confirmar los resultados. La principal diferencia entre resultados estructurales calculados con los potenciales ensayados radica en la mejor reproducción por parte del modelo BKS, obteniéndose un mayor nivel de precisión si se compara con el modelo PMM.

\subsubsection{Difusión}

El coeficiente de auto-difusión de moléculas adsorbidas en materiales posrosos, puede entenderse como una medida de la evolución de la posición tridimensional del adsorbato dentro de la estructura cristalina como consecuencia exclusiva de su energía térmica. A partir del método de la DM se han calculado los coeficientes de difusión de dos adsorbatos: propano y propeno para las estructuras seleccionadas. Los coeficientes de difusión intracristalina se han calculado a partir de las estructuras modeladas con los potenciales BKS y PMM descritos anteriormente.

La coincidencia entre los tamaños de las ventanas de las zeolitas y los diámetros cinéticos de los adsorbatos hace necesario que el tiempo de simulación sea extremadamente largo, esto con el fin de obtener un cierto nivel de confianza estadística en los resultados. Para el caso de los sistemas descritos, los tiempos de simulación llegan a los $100 \mathrm{~ns}$, esto es $10^{8}$ pasos de simulación. La dinámica del propano/propeno se reduce a saltos que ocurren despues de tiempos de residencia en los poros relativamente largos, debido a la barrera que presentan las ventanas 


\begin{tabular}{lccc}
\hline \multicolumn{4}{c}{ Diametros Críticos (̊̊) } \\
Estructuras & LX & LY & LZ \\
\hline Propano & 4,0 & 4,5 & 6,6 \\
Propeno & 4,0 & 4,2 & 6,6 \\
Etano & 3,8 & 4,1 & 4,8 \\
Metano & 3,8 & 4,1 & 3,9 \\
\hline
\end{tabular}

Tabla 4.6: Dimensiones de hidrocarburos, calculadas a partir de las proyecciones de las moléculas en diferentes planos. $L X, L Y$ y $L Z$ hace referencia a las dimensiones de las proyecciones de las moléculas en los planos $X Z, X Y$ y $Y Z$, respectivamente.

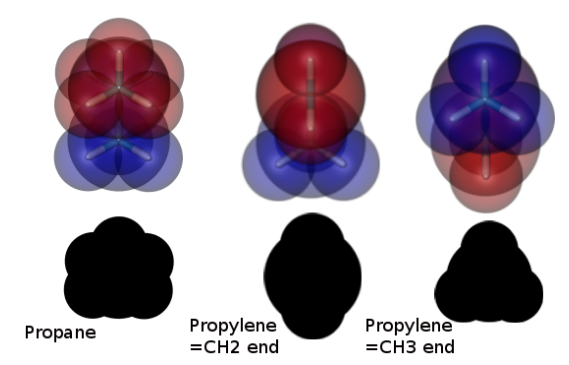

Figura 4.4: Representación de las proyecciones de los grupos $\mathrm{CH} 3$ y $\mathrm{CH} 2$ terminales en moléculas de propano y propeno.

que comunican los poros. Para tener una idea del tamaño cinético de los adsorbatos, la tabla 4.6 muestra las dimensiones de algunos hidrocarburos. Las dimensiones han sido calculadas siguiendo el esquema de Rohrbaugh y Jurs, ${ }^{160}$ que asigna las dimensiones de las moléculas a partir de las proyecciones en diferentes planos (Ver Fig. 4.4).

La tabla 4.7 muestra los coeficientes de difusión para propano y propeno en las dos estructuras y con los dos potenciales interatómicos usados para representar las interacciones en las zeolitas. A las temperaturas de simulación más bajas, 300 y $400 \mathrm{~K}$, el nivel de incertidumbre en el desplazamiento medio de las moléculas es muy bajo. El error estandar del DMC es de dos y un orden de magnitud para las temperaturas de 300 y $400 \mathrm{~K}$, repsectivamente. Por este motivo, se hace necesario acelerar la dinámica de los adsorbatos, para lo cual incrementamos la excitación térmica a niveles de 600 y $800 \mathrm{~K}$, condiciones a las cuales la dinámica de saltos entre poros adyacentes se incrementa notablemente, permitiendo el cálculo de DMC con incertidumbre estadística mucho más baja. A $800 \mathrm{~K}$, el error estandar llega a ser tres órdenes de magnitud menor al coeficiente de difusión calculado, aunque la movilidad entre poros adyacentes es aún baja como puede observarse 


\begin{tabular}{l|cccc}
\hline \multicolumn{5}{c}{$D_{B K S}\left[\frac{m^{2}}{s}\right]$} \\
\hline Si-CHA/Propeno & $5,2 \times 10^{-11}$ & $8,8 \times 10^{-11}$ & $1,0 \times 10^{-10}$ & $1,6 \times 10^{-10}$ \\
Si-CHA/Propano & $\sim 10^{-14}$ & $1,6 \times 10^{-14}$ & $5,12 \times 10^{-14}$ & $9,6 \times 10^{-12}$ \\
Si-SAS/Propeno & -N/A- & -N/A- & $8,8 \times 10^{-11}$ & $1,1 \times 10^{-10}$ \\
\hline \multicolumn{5}{c}{$D_{P M M}\left[\frac{m^{2}}{s}\right]$} \\
Si-CHA/Propeno & $2,41 \times 10^{-11}$ & $4,6 \times 10^{-11}$ & $1,31 \times 10^{-10}$ & $2,75 \times 10^{-10}$ \\
Si-CHA/Propano & $\sim 10^{-14}$ & $1,5 \times 10^{-14}$ & $6,0 \times 10^{-12}$ & $1,1 \times 10^{-11}$ \\
Si-SAS/Propeno & -N/A- & -N/A- & $7,1 \times 10^{-11}$ & $1,1 \times 10^{-10}$ \\
\hline
\end{tabular}

Tabla 4.7: Constantes de auto-difusión para propano/propeno a las condiciones indicadas usando los potenciales BKS y PMM.

en la escala de la figura 4.5.

Los coeficientes de difusión para propeno muestran valores más altos, en comparación con el propano, a pesar de la similitud en el tamaño de la molécula con las ventanas entre poros. Coeficientes de difusión para propeno están en el rango $10^{-11}-10^{-10}\left[\frac{m^{2}}{\mathrm{~s}}\right]$ en el intervalo de temperaturas $300-800 \mathrm{~K}$. La movilidad del propeno en la estructura Si-CHA presenta coeficientes de difusión órdenes de magnitud mayores a los obtenidos para el propano: $\left[D_{\text {Propeno }} \sim\right.$ $\left.10^{-11} / D_{\text {Propano }} \sim 10^{-14}\right]$ a $300 \mathrm{~K}$, y $\left[D_{\text {Propeno }} \sim 10^{-10} / D_{\text {Propano }} \sim 10^{-12}\right]$ a la temperatura más alta, $800 \mathrm{~K}$.

Los coeficientes de difusión caclulados para sistemas modelados con el potencial PMM muestran un comportamiento similar, con magnitudes de igual orden a los calculados para el modelo BKS, aunque un pequeño aumento en los coeficientes es visible a las temperaturas más altas (Ver fig. 4.5 y Tabla 4.7). La influencia del modelo interatómico usado no es visible a las temperaturas más bajas, ya que los coeficientes de difusión no pueden calcularse con un alto grado de confianza estadística, debido al casi inexistente movimiento entre poros a estas condiciones. Aún a $600 \mathrm{~K}$ la pendiente de la curva DMC es positiva, mientras que la obtenida con el modelo BKS es totalmente plana. A esta temperatura hay una diferencia entre coeficientes de difusión de dos órdenes de magnitud $\left[D_{\text {Propane }}^{B K S-600} \sim 10^{-10} / D_{\text {Propane }}^{P M M-600} \sim 10^{-12}\right]$, aunque las desviaciones estandar de los coeficientes son de la misma magnitud que los valores obtenidos. El orden de magnitud de los coeficientes de auto-difusion a $800 \mathrm{~K}$ es practicamente igual, independientemente del modelo utilizado, aunque los valores obtenidos para el potencial PMM es dos veces mayor al del BKS. Las dimensiones de las ventanas obtenidos con los potenciales tienen por lo tanto un impacto visible en los coeficientes de difusión, el cual se hace más evidente a la temperatura de simula- 

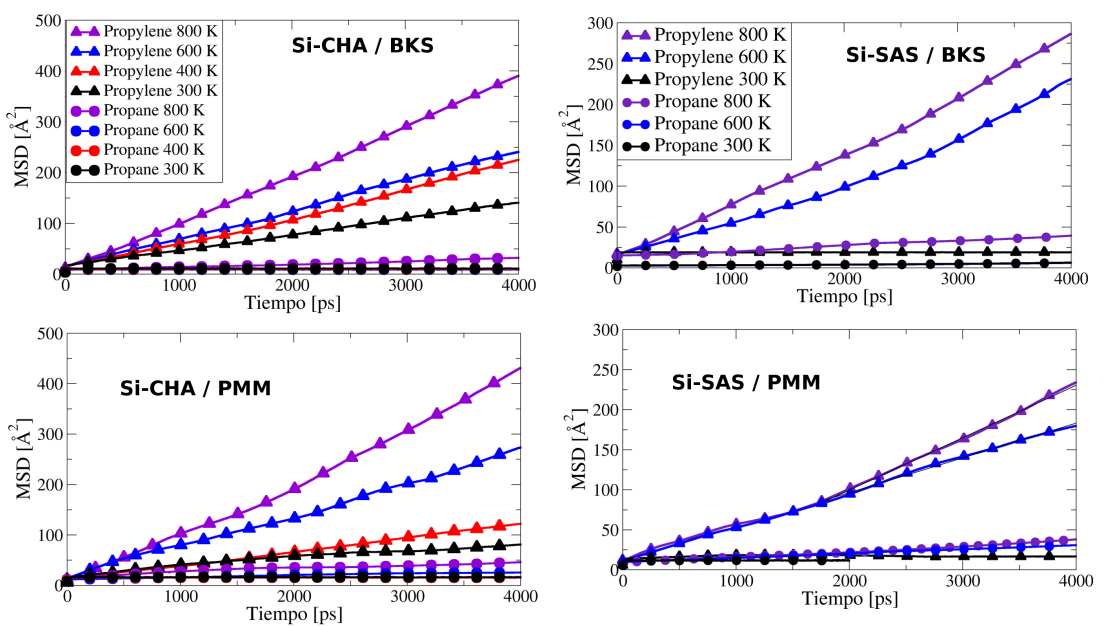

Figura 4.5: Desplazamientos medios al cuadrado para propano y propeno en Si-CHA y Si-SAS a partir de trayectorias de DM. Curvas obtenidas con los potenciales BKS y PMM FFs at 300, 400, 600 y $800 \mathrm{~K}$. La carga de adsorbato en todos los casos es de 0.5 moleculas por celda unidad.

ción más alta, $800 \mathrm{~K}$, condición a la cual existe un aceleramiento de la dinámica de los adsorbatos que permite tener mayor grado de confianza en los resultados obtenidos.

$\mathrm{Al}$ igual que para la zeolita $\mathrm{Si}-\mathrm{CHA}$, se han calculado los coeficientes de difusión a diferentes temperaturas para el sistema propano/propeno-Si-SAS. La Figura 4.5 muestra los DMC de los adsorbatos, a partir de los cuales se han calculado los coeficientes de difusión.

Los coeficientes de difusión obtenidos con los potenciales PMM y BKS están en el mismo rango de magnitudes, tal como se muestra en la tabla 4.7. Una característica sobresaliente de la estructura Si-SAS es su caracter unidimensional, lo que hace que la dinámica de las moléculas se vea forzada en la dirección de los canales. Este efecto es visible a través de los coeficientes de difusión, los cuales, a pesar de la mayor amplitud del diámetro crítico de ventana en la Si-SAS comparada con la de Si-CHA, son en general de menor magnitud que los obtenidos para Si-CHA. En este aspecto, la conectividad tridimensional entre poros de la estructura de tipo CHA, y por consiguiente la mayor probabilidad para efectuar traslaciones a poros contiguos, producirá un mayor valor en los coeficientes de difusión. 


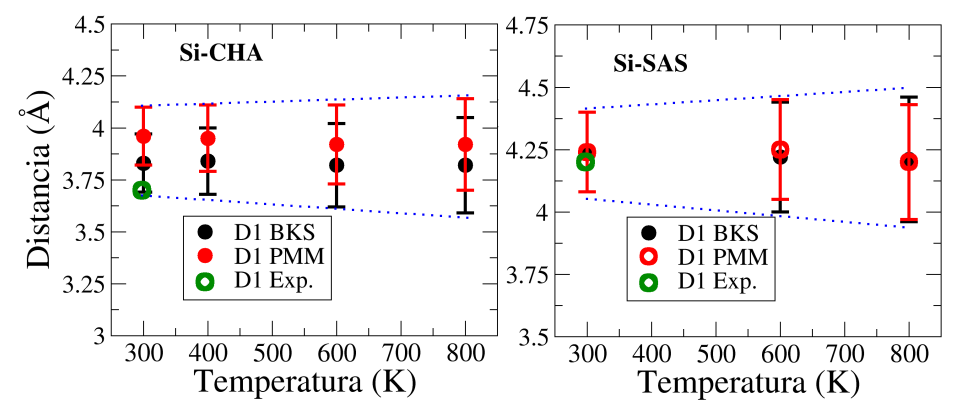

Figura 4.6: Variación del diámetro crítico (D1) de ventana en las zeolitas Si-CHA y Si-SAS respecto a la temperatura. Las barras muestran las fluctuaciones de las ventanas respecto a los valores medios. El círculo verde representa el valor experimental para cada estructura.

\subsection{Conclusiones}

En este capítulo se ha estudiado la influencia del potencial interatómico usado para modelar la interacciones de la estructura cristalina. El efecto del potencial se analizó empleando dos modelos diferentes, ampliamente utilizados en cálculos atomísticos, con el objetivo de resaltar el efecto del potencial sobre los parámetros estructurales del material estudiado y sobre la movilidad de las moleculas adsorbidas.

La metodología desarrollada en este capítulo, se basa en la identificación de factores estructurales asociados al tipo de modelo utilizado para la representación de las interacciones internas de la red. Incialmente se estudia la respuesta de los potenciales seleccionados, BKS y PMM, ante la variación de los parámetros estructurales de la red con la temperatura. El efecto de variación negativa del volumen respecto a la temperatura es capturado por los dos modelos, con una mejor representación de los resultados experimentales obtenida a través del potencial BKS. Si bien el comportamiento a altas temperaturas no se acopla perfectamente a los datos experimentales para ninguno de los potenciales, la desviación es menor al usar el potencial BKS.

En segundo lugar, se estudió la respuesta de los diámetros críticos de las ventanas entre poros en función de la temperatura, y su desviación con respecto a los datos cristalográficos experimentales. Los diámetros de las ventanas y sus fluctuaciones fueron obtenidos a partir de funciones de distribución entre pares, tomando en cuenta que las estructuras presentaban flexibilidad total.

Las desviaciones del diámetro de ventana para las zeolitas Si-CHA y Si-SAS 


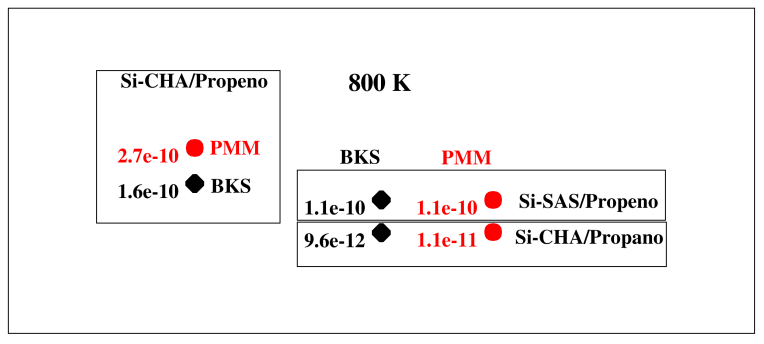

Figura 4.7: Comparativa del coeficiente de difusión de propeno y propano en las zeolitas SiCHA y Si-SAS a $800 \mathrm{~K}$. El cuadro de la izquierda compara los coeficientes de difusión del propeno obtenidos utilizando los potenciales BKS y PMM. En los recuadros de la izquierda se muestran los coeficientes de difusión del propano en las dos estructuras y calculados con cada uno de los potenciales.

se muestran graficamente en la figura 4.6. Las fluctuaciones van desde 0.14 hasta $0.23 \AA$ para Si-CHA, y desde 0.14 hasta $0.22 \AA$ para Si-SAS. Las variaciones en el diámetro crítico medio no se ven afectadas por la temperatura de manera apreciable, sin embargo, las fluctuaciones de las ventanas muestran un incremento notable, y por consiguiente a condiciones de temperatura alta se tendrá una barrera difusiva menor, como consecuencia de la excitación térmica. Este efecto se sumará a la excitación propia de los grados de libertad translacionales para los adsorbatos, con lo cual se obtiene un aumento en los coeficientes de difusión correspondientes.

La figura 4.7 permite ver la influencia del potencial en los coeficientes de difusión $\left(\mathrm{m}^{2} / \mathrm{s}\right)$ para el propano y propeno. El coeficiente de difusión del propeno calculado con el potencial BKS, el cual predice un diámetro crítico de ventana menor al obtenido con el potencial PMM, es igualmente menor en magnitud al calculado con el potencial PMM. Sin embargo, la diferencia de los coeficientes de difusión, en este caso comparando los valores a $800 \mathrm{~K}$ para propeno en Si-CHA, no son en extremo diferentes. Igual situación se da para el propano, obteniendose coeficientes virtualmente iguales independientemente del potencial utilizado. 


\title{
Capítulo 5
}

\section{Difusión de Hidrocarburos en Zeolitas}

\begin{abstract}
La técnica de la Dinámica Molecular es utilizada en este capítulo para estudiar la movilidad de metano, etano, propano y propeno en tres estructuras cristalinas de tipo IHW, ITE y LTA. Estas estructuras están formadas por poros conectados por anillos de 8 unidades tetraédricas de óxido de silicio, con diferente dimensionalidad y cuyos diámetros críticos de ventana son del mismo orden de magnitud que los diámetros cinéticos de los adsorbatos. La finalidad de este estudio es el de describir la dinámica de los adsorbatos, y mostrar su dependencia respecto a las características topológicas de las estructuras estudiadas.
\end{abstract}

En el presente capítulo se reportan los resultados obtenidos al emplear la técnica de la DM para el estudio de la movilidad de metano, etano, propano y propeno adsorbido en tres estructuras zeolíticas con anillos de 8 unidades tetraédricas de $\mathrm{SiO}_{4}$ (8-ring). El primero de los objetivos es el de describir la dependencia de la movbilidad de los adsorbatos respecto a la temperatura. Pare este fin se han seleccionado tres estructuras, puramente silíceas, de tipo LTA ${ }^{12}$, ITE ${ }^{13}$ e IHW. ${ }^{11}$

La selección de estructuras puramente silíceas obedece a la necesidad de simplificación de las interacciones entre los adsorbatos y la red cristalina con el fin de resaltar los efectos puramente topológicos sobre la movilidad de los adsorbatos. En el caso de zeolitas con iones intercambiados, los efectos de la red provendrían de dos fuentes: inicialmente de la conformación topológica de la red y en segundo lugar de las interacciones provenientes de los cationes presentes.

De las estructuras seleccionadas, la zeolita Si-LTA (zeolita ITQ-29) es probablemente la más estudiada, tanto computacional ${ }^{161-166}$ como experimentalmen- 


\begin{tabular}{lcccccccc}
\hline Zeolita & $\mathbf{a}[\AA]$ & $\mathbf{b}[\AA]$ & $\mathbf{c}[\AA]$ & $\alpha=\beta, \gamma[\mathbf{G r a d}]$ & Vol $\left[\AA^{3}\right]$ & Celda Sim. & Atomos & Dim. \\
\hline (ITQ-29) LTA $^{\mathbf{1 2}}$ & 11.92 & 11.92 & 11.92 & 90.0 & 1693.2 & $2 \times 2 \times 2$ & 576 & $3 \mathrm{D}$ \\
(ITQ-32) IHW $^{\mathbf{1 1}}$ & 13.70 & 24.02 & 18.20 & 90.0 & 6064.6 & $2 \times 1 \times 1$ & 672 & 2D \\
${\text { (ITQ-3) } \text { ITE }^{\mathbf{1 3}}}^{20.75}$ & 9.80 & 20.01 & 90.0 & 4071.1 & $2 \times 2 \times 1$ & 768 & 2D (1D)
\end{tabular}

Tabla 5.1: Estructuras zeolíticas: parámetros de red y volumen de celda.

te, ${ }^{140,141,167,168}$ debido a su variada utilización a nivel industrial y a la alta simetría de su red cúbica. ${ }^{12}$ Con base en el anterior argumento, la estructura Si-LTA es un candidato ideal para evaluar la pertinencia de la metodología planteada, la cual se basa en el método de la dinámica molecular.

\subsection{Estructuras Seleccionadas}

Se han seleccionado tres estructuras zeolíticas, compuestas enteramente por unidades tetraédricas silíceas, de tipo LTA, IHW e ITE. La tabla 5.1 y la Figura 5.1 proporcionan detalles estructurales de las zeolitas utilizadas como modelos: parámetros de red, volúmenes, tamaños de las celdas de simulación, número de átomos, así como la dimensionalidad de los canales intracristralinos.

La zeolita ITQ-29 es la contraparte puramente silícea de los materiales con tipo de red LTA, y puede definirse estructuralmente como una red de cavidades esféricas (celdas alfa), interconectadas tridimensionalmente, de longitud aproximada a $11.4 \AA$ conectadas por seis ventanas de tamaño pequeño (8-ring) con diámetro efectivo de $4.1 \AA$.

Alternativamente, la estructura LTA puede describirse como una red formada por celdas de sodalita (celdas beta) conectadas entre si por sus caras cuadradas planas, formando así anillos dobles de dimensión 4-ring. Esta estructura ofrece la posibilidad de difusión paralela a los tres ejes cristalográficos.

La topología de la zeolita ITQ-32 (estructura IHW) se compone de canales unidireccionales a lo largo del eje cristalográfico a, con aperturas de $3,5 \times 4,3$ Å. El sistema poroso resultante está cruzado por canales relativamente cortos con ventanas de tipo 12-ring, el cual conecta canales 8-ring vecinos a lo largo del eje c. Estos canales tienen un diámetro de $6.3 \AA$ y una longitud de $16.2 \AA .{ }^{11} \mathrm{La}$ zeolita ITQ-32 ha sido objeto de estudios experimentales para probar su posible utilización en procesos de separación de mezclas olefina/parafina, específicamente para su utilización en la separación cinética de moléculas de tamaño similar, caso específico de la separación de propano/propeno. ${ }^{20}$

La zeolita ITQ-3, de estructura ITE, está formada por una red bidimensional de canales rectos conectados por ventanas 8-ring orientadas a lo largo de los ejes cristalográficos b y c, grupo espacial $\mathrm{Cmcm}$ y celda unidad ortorómbica. La in- 


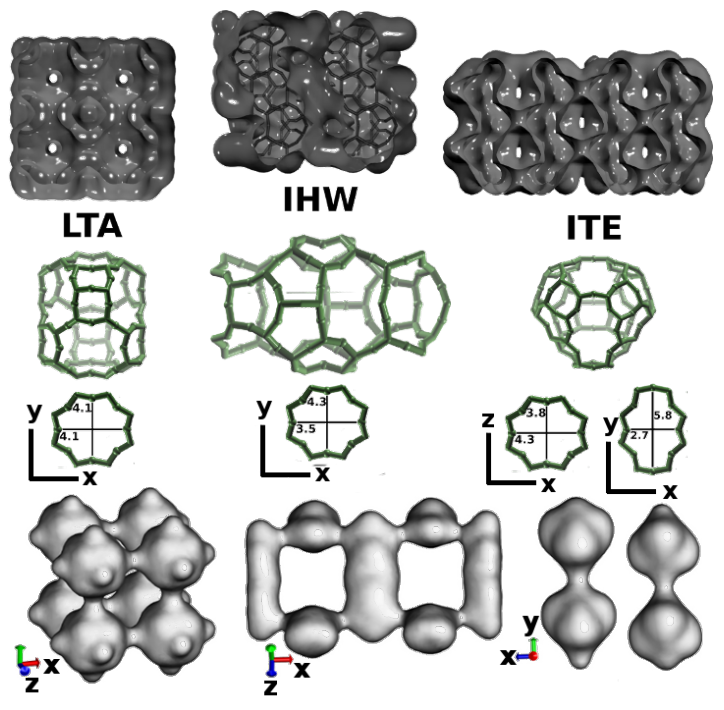

Figura 5.1: Representación de los mapas de probabilidad de ocupación (arriba), celdas, canales y tamaños de ventana (centro), y mapas volumétricos de la densidad de probabilidad del $\mathrm{CH}_{4}$ adsorbido en las diferentes zeolitas (abajo).

tersección de los canales generan cuatro cavidades por celda unidad, conectadas por ventanas 8-ring de diferentes dimensiones. ${ }^{13}$ La marcada diferencia en los tamaños de dichas ventanas (ver Fig. 5.1) permite la difusión de adsorbatos unicamente a lo largo del eje cristalográfico b, lo que hace que esta estructura sea efectivamente un arreglo unidimensional de celdas conectadas por ventanas de tamaño molecular. Al igual que la zeolita ITQ-32, esta estructura también ha sido estudiada experimentalmente para la adsorción de hidrocarburos pequeños (C1C4) y como posible tamiz molecular para separaciones de moléculas de tamaño similar. $^{21}$

Las zeolitas descritas anteriormente presentan como característica común el poseer poros conectados por ventanas con 8 unidades de $\mathrm{SiO}_{4}$, pero las configuraciones de las ventanas dependen de las características topológicas específicas para cada estructura. Las dimensiones y formas de las ventanas estarán dictadas por los ángulos intrínsecos $\mathrm{SiOSi}-\mathrm{OSiO}$ y los dihedros $\mathrm{SiOSiO}$. Las dimensiones de las ventanas se calcularon de acuerdo a las recomendaciones de la IZA de sustraer dos veces el radio de van der Waals para el oxígeno. ${ }^{159}$ Como comparación hemos calculado los diámetros cinéticos del metano, etano, propeno y propano siguiendo el método desarrollado por Rohrbaugh y Jurs. ${ }^{160}$ Los datos obenidos se muestran en la Tabla 5.2. 


\begin{tabular}{|c|c|c|c|}
\hline \multicolumn{4}{|c|}{ Diametros Críticos $(\AA)$} \\
\hline Estructuras & $\mathbf{L X}$ & $\mathbf{L Y}$ & $\mathbf{L Z}$ \\
\hline Propano & 4.0 & 4.5 & 6.6 \\
\hline Propeno & 4.0 & 4.2 & 6.6 \\
\hline Etano & 3.8 & 4.1 & 4.8 \\
\hline Metano & 3.8 & 4.1 & 3.9 \\
\hline
\end{tabular}

Tabla 5.2: Diámetros críticos para metano, etano, propeno y propano. $L X, L Y$ y $L Z$ hace referencia a la longitud de las sombras resultantes de la proyección de las moléculas respecto a los planos $X Z, X Y$ y $Y Z$, respectivamente. ${ }^{160}$

\subsection{Metodología}

El método de DM es utilizado para simular la movilidad intra-poro e interporo de metano, etano, propano y propeno adsorbido en zeolitas de pura sílica. Los cálculos se han levado a cabo utilizando el código paralelo de propósito general DL_POLY 2.20. ${ }^{156}$

Como regla general hemos implementado las simulaciones permitiendo la flexibilidad de las estructuras cristalinas, ya que como se mostró en el capítulo anterior existe una dependencia de la difusión de adsorbatos con respecto a las fluctuaciones de las ventanas. La condición de flexibilidad estructural nos permite hacer uso del ensamble microcanónico, NVE, sin necesidad de implementar un termostato para el control de la temperatura.

Los cálculos de probabilidades de ocupación han sido efectuados a partir de la generación de histogramas, obtenidos a su vez de las trayectorias a las condiciones específicadas de temperatura y carga de adsorbato. ${ }^{68,169} \mathrm{El}$ tamaño de las celdas de simulación se efectuó por medio del escalamiento de las celdas unidad, tal como se especifica en la tabla 5.1. La integración de las ecuaciones de movimiento de Newton se lleva a cabo utilizando el algoritmo de Velocity-Verlet, con un tamaño de paso de $1 \mathrm{fs}$.

La carga de adsorbato se define como 4 moléculas por celda de simulación, para todos los sistemas. Este nivel de carga permite relativamente bajo permite obtener resultados de difusividad dependientes en mayor grado de la topología de las estructuras, y no de la interacción entre especies que se presentaría al tener cargas más elevadas.

\subsubsection{Detalles de Cálculo}

Todos los sistemas especificados se evalúan en el colectivo NVE, en el rango de temperaturas entre 300-1200 K. Inicialmente los sistemas se pre-equilibran 
por un período de hasta $150 \mathrm{ps}$, ajustando las velocidades de las partículas del sistema cada 5 fs hasta que se obtiene una distribución de temperatura estable y ajustada a la temperatura objetivo. Un segmento posterior de equilibración de hasta 200 ps se utiliza antes de iniciar la toma de datos, permitiéndose de esta forma que las fluctuaciones de temperatura alcancen un nivel estadisticamente confiable. El muestreo de las trayectorias finales se inicia despues de este paso de pre-equilibración en el colectivo NVE, con tiempos de simulación de $10 \mathrm{~ns}$ para metano, $20 \mathrm{~ns}$ para etano, $80 \mathrm{~ns}$ para propeno y $100 \mathrm{~ns}$ para propano. El análisis de las trayectorias se lleva a cabo a partir de los archivos generados por DL_POLY con las posiciones y velocidades de las partículas del sistema, utilizando la relación de Einstein,

$$
2 t D=\frac{1}{N_{t}}\left\langle\left|r\left(t+t_{0}\right)-r(t)\right|^{2}\right\rangle
$$

donde, tal como se especificó en el capítulo 2 (Eq. 10), $r$ corresponde a la posición del centro de masa del adsorbato, $N_{t}$ hace referencia al número de grados de libertad translacional de dicha molécula, y el símbolo \langle\rangle representa el promedio evaluado en el colectivo NVE. El factor $N_{t}$ es de importancia fundamental, ya que involucra la influencia de la dimensionalidad de la estructura cristalina respecto a la movilidad del adsorbato (ver Tabla 5.1 y Figura 5.1), y se define para cada estructura como: $N_{t}^{L T A}=3, N_{t}^{I H W}=2$ y $N_{t}^{I T E}=1$. La dependencia del coeficiente de difusión con la temperatura se estima utilizando la ecuación de Arrhenius, detallada también en el capítulo 2 (Eq. 8). Cabe anotar que las energías de activación obtenidas para los adsorbatos $\mathrm{C} 3$ deben ser vistos como valores puramente cualitativos, debido a su limitada movilidad en las estructuras estudiadas, lo que dificulta obtener valores de coeficientes de difusión con un alto grado de confianza estadística, especialmente a bajas temperaturas.

\subsubsection{Superficie de Energía Potencial}

La función que describe la energía potencial de los sistemas involucra tres términos principales: La energía potencial de la zeolita $\left(V^{Z e o}\right)$, la energía potencial del adsorbato $\left(V^{A d s}\right)$, y la contribución intermolecular $\left(V^{Z e o-A d s / A d s-A d s}\right)$.

$$
V^{\text {Total }}=V^{Z e o}+V^{\text {Guest }}+V^{Z e o-A d s / A d s-A d s}
$$

La energía potencial de la red cristalina se representa en la siguiente forma: un término Coulómbico que integra la contribución electrostática de largo alcance, y una función que describe la repulsión-dispersión entre pares de partículas,

$$
V^{Z e o}=V^{Z e o-V d w}+V^{Z e o-C o u l}
$$




\begin{tabular}{ll}
\hline$S i$ [e] +2.4 & \\
$O[\mathbf{e}]-1.2$ & \\
\hline$A_{S i-O}[\mathrm{ev}]$ & 18003.7572 \\
$A_{O-O}[\mathrm{ev}]$ & 1388.7730 \\
$\rho_{S i-O}[\AA]$ & 0.205205 \\
$\rho_{O-O}[\AA]$ & 0.362319 \\
$C_{S i-O}\left[\mathrm{ev \AA}{ }^{6}\right]$ & 133.5381 \\
$C_{O-O}\left[\mathrm{ev \AA}{ }^{6}\right]$ & 175.0000 \\
\hline
\end{tabular}

Tabla 5.3: Parámetros para el potencial BKS

Para la evaluación de las interacciones electrostáticas se ha utilizado el algoritmo Particle Mesh Ewald. ${ }^{136}$ La descripción de las interacciones de corto alcance $\left(V^{Z e o-V d W}\right)$ se lleva a cabo usando el modelo de Born-Mayer para sólidos iónicos de van Beest et al. (BKS), ${ }^{104}$ el cual se ha descrito en detalle en el capítulo 3. El potencial BKS se aplica solo a las interacciones entre los pares oxígeno-oxígeno y oxígeno-silicio. Este potencial (Ver ecuación 5.4 y parámetros en Tabla 5.3) se divide en dos términos: una contribución repulsiva, dada por el término exponencial, y un término atractivo-dispersivo.

$$
V_{B K S}^{Z e o-V d W}=A_{i j} \exp \left(\frac{-r_{i j}}{\rho_{i j}}\right)-\frac{C_{i j}}{r^{6}}
$$

El potencial intramolecular para los hidrocarburos integra interacciones entre pares, interacciones angulares entre tres partículas e interacciones descritas por los diedros formados entre cuatro partículas. Los parámetros se describen en la referencia original de Oie et al. ${ }^{158}$ Finalmente, el potencial de Lennard-Jones (126) es usado para la descripción de las interacciones intermoleculares adsorbatozeolita y adsorbato-adsorbato.

\subsection{Difusión en Zeolitas}

\subsubsection{Dinámica en la Zeolita Si-LTA}

Los perfiles de desplazamiento medio al cuadrado a diferentes temperaturas para metano, etano, propeno y propano adsorbido en la zeolita ITQ-29 se muestran en la Figura 5.2, y coeficientes de auto-difusión $D_{s}$ calculados usando la ecuación de Einstein-Smoluchowsky se presentan en las Tablas 5.4 y 5.4. El efecto global de la temperatura en el desplazamiento de los adsorbatos se observa 


\begin{tabular}{ccc} 
& \multicolumn{2}{c}{ Coeficientes de Difusión $D_{s}\left(\frac{m^{2}}{s}\right)$} \\
\hline $\mathrm{T}(\mathrm{K})$ & $\mathbf{C H}_{\mathbf{4}}$ & $\mathbf{C}_{\mathbf{2}} \mathbf{H}_{\mathbf{6}}$ \\
\cline { 2 - 3 } 300 & $5,9 \times 10^{-9}$ & $4,8 \times 10^{-10}$ \\
600 & $6,1 \times 10^{-9}$ & $4,3 \times 10^{-10}$ \\
900 & $9,5 \times 10^{-9}$ & $6,6 \times 10^{-10}$ \\
1200 & $1,5 \times 10^{-8}$ & $1,7 \times 10^{-9}$ \\
\hline & $3,0 \times 10^{-10}(\mathrm{MD} \mathrm{300} \mathrm{K})^{59}$ & $1,0 \times 10^{-11}-1,0 \times 10^{-7}(\operatorname{Exp} 300 \mathrm{~K})^{167}$ \\
& $1,0 \times 10^{-9}(\mathrm{MD} \mathrm{300} \mathrm{K})^{162}$ & $2,09 \times 10^{-10}(\operatorname{Exp~300~K})^{140}$ \\
& $1,42 \times 10^{-10}\left(\operatorname{Exp~300~K)^{140}}\right.$ & $1,2 \times 10^{-10}(\mathrm{MD} \mathrm{750} \mathrm{K})^{165}$ \\
& $1,0 \times 10^{-9}(\mathrm{MD} \mathrm{600} \mathrm{K})^{165}$ & \\
\hline
\end{tabular}

Tabla 5.4: Coeficientes de Difusión experimental y calculados para metano y etano en la zeolita ITQ-29. Arriba: resultados obtenidos en el presente estudio. Abajo: datos publicados de valores experimentales y calculados en otros trabajos.

en forma de aumento en las pendientes de las curvas de desplazamiento. También puede observarse la fuerte dependencia del desplazamiento inter-poro con respecto al tamaño del adsorbato. Las curvas de MSD para metano fluctuan desde 4000 $\AA^{2}$ hasta algo más de $10000 \AA^{2}$ en el rango de temperaturas y tiempo representados en la figura 5.2, con la característica dependencia lineal del desplazamiento con respecto al tiempo, lo cual es un buen indicador de un regimen de difusion no anómalo.

La longitud de los desplazamientos en este sistema sugiere además que la trayectoria de las moléculas alcanza varias veces el tamaño de la celda de simulación, con lo cual se genera un alto grado de certidumbre estadística para el cálculo de los coeficientes de difusión.Los coeficientes de difusión obtenidos son comparables a los valores experimentales reportados por Hedin et al. ${ }^{140} \mathrm{y}$ con cálculos previos llevados a cabo via DM. ${ }^{59,162,165}$

El comportamiento de las curvas de desplazamiento se altera de manera notoria al incrementarse el tamaño del adsorbato. Para etano, el rango de los desplazamientos varía entre $400 \AA^{2}$ a $300 \mathrm{~K}$ hasta $2000 \AA^{2}$ a $1200 \mathrm{~K}$ en la escala de tiempo representado en la figura 5.2. Al igual que en el caso del metano, las MSDs presentan un comportamiento lineal, con pendiente positiva, indicando que la movilidad del adsorbato abarca distancias por encima del tamaño de la celda de simulación, reforzando el argumento de la movilidad entre celdas cruzando las barreras entrópicas ejercidas por las ventanas.

Cabe destacar que existe un aumento considerablemente mayor de pendiente en los MSDs entre las temperaturas de $900 \mathrm{~K}$ y $1200 \mathrm{~K}$, lo que sugiere un comportamiento no compatible con el modelo de Arrhenius para este adsorbato a altas temperaturas. Este comportamiento se hace patente en la figura 5.3, la cual 


\begin{tabular}{ccc} 
& \multicolumn{2}{c}{ Coeficientes de Difusión $D_{s}\left(\frac{\mathrm{m}^{2}}{\mathrm{~s}}\right)$} \\
\hline $\mathrm{T}(\mathrm{K})$ & $\mathbf{C}_{\mathbf{3}} \mathbf{H}_{\mathbf{6}}$ & $\mathbf{C}_{\mathbf{3}} \mathbf{H}_{\mathbf{8}}$ \\
\cline { 2 - 3 } 300 & $1,4 \times 10^{-11}$ \\
600 & $5,6 \times 10^{-11}$ & $4,0 \times 10^{-12}$ \\
900 & $9,1 \times 10^{-11}$ & $1,65 \times 10^{-11}$ \\
1200 & $1,5 \times 10^{-10}$ & $3,8 \times 10^{-11}$ \\
\hline & $4,7 \times 10^{-15}\left(\operatorname{Exp~300~K)^{140}}\right.$ & $1,5 \times 10^{-12}(\mathrm{MD} 750 \mathrm{~K})^{170}$ \\
& $5,0 \times 10^{-14}(\operatorname{Exp} 300 \mathrm{~K})^{141}$ & $2,0 \times 10^{-14}(\mathrm{dcTST} 600 \mathrm{~K})^{165}$ \\
\hline
\end{tabular}

Tabla 5.5: Coeficientes de Difusión experimental y calculados para propeno y propano en la zeolita ITQ-29. Arriba: resultados obtenidos en el presente estudio. Abajo: datos publicados de valores experimentales y calculados en otros trabajos.
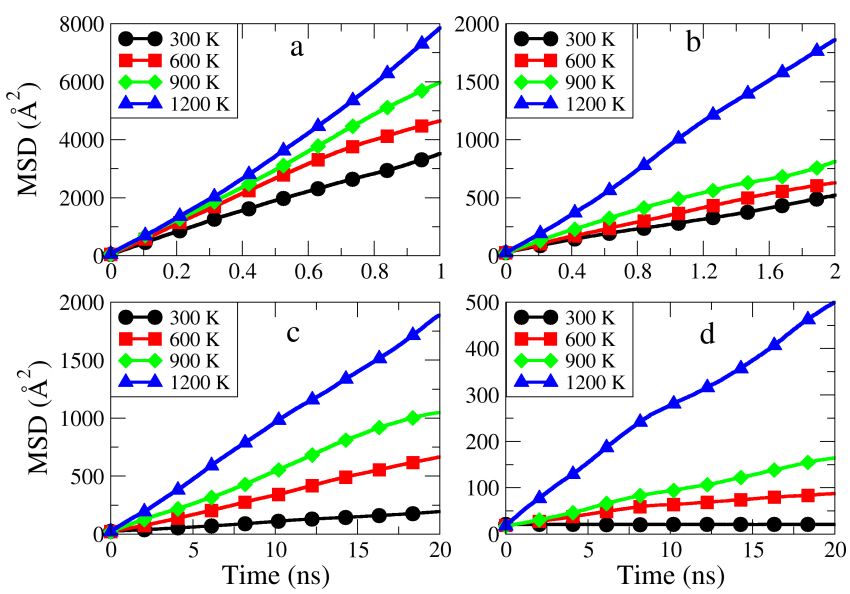

Figura 5.2: Perfiles MSD para (a) metano, (b) etano, (c) propeno y (d) propano adsorbido en la zeolita Si-LTA a diferentes temperaturas. 
muestra la dependencia no lineal entre los coeficientes de difusión y la temperatura para metano y etano. Las energías de activación calculadas muestran un valor bajo para el metano y etano, entre 2.5 y $2.9 \mathrm{~kJ} / \mathrm{mol}$ respectivamente.

La figura 5.4 representa la densidad de ocupación, $p(z)$, a lo largo del eje $\mathrm{z}$ para $\mathrm{CH}_{4}, \mathrm{C}_{2} \mathrm{H}_{6}, \mathrm{C}_{3} \mathrm{H}_{6}$ y $\mathrm{C}_{3} \mathrm{H}_{8}$ en el interior de la zeolita ITQ-29 en el rango de temperatura 300-1200 K. Los extremos representados en la coordenada $\mathrm{Z}$ ( $Z=$ $0, Z \sim 12 \AA$ ) corresponden a las ventanas de 8-ring que conectan celdas vecinas, mientras que el punto medio $(Z \simeq 6 \AA$ ) correspondería al centro de una cavidad.

A la temperatura de simulación de $300 \mathrm{~K}$, el metano y etano ocupan zonas bien definidas de la cavidad, en posiciones con máximos relativos en las gráficas de densidad de población. el efecto del aumento de temperatura se ve como una distribución más uniforme en la densidad de ocupación, con máximos locales desplazándose en favor de una distribución menos localizada. Este comportamiento sugiere que las barreras energéticas intra-cavidad se hacen menos importantes permitiendo a la molécula desplazarse libremente por el interior de las cavidades, aumentando así la probabilidad de ubicarse en posiciones favorables y con la cantidad de energía necesarias para cruzar a través de las barreras energéticas creadas por las ventanas 8-ring. El panorama es basicamente igual para etano, con la diferencia de que las posiciones preferenciales del centro de masa en el interior de la cavidad están un poco más alejadas de las paredes de la red cristalina.

En el caso del propeno se observa un cierto nivel de orden a la temperatura de $300 \mathrm{~K}$, con dos regiones preferenciales de adsorción alrededor del centro de la cavidad, los cuales desaparecen y dejan paso a una distribución uniforme a temperaturas más altas. En el caso de propano, las distribuciones del centro de masa se ubican preferencialmente en el centro de la cavidad, y no se observan diferencias notorias con el aumento de la excitación térmica. En todos los casos, los valores extremos de las distribuciones de población, $P(0)$ y $P(12)$, son efectivamente diferentes de cero, con lo cual se confirma la existencia de eventos de cruce entre cavidades.

Los diagramas de Arrhenius para los diferentes adsorbatos en Si-LTA se muestran en la Figura 5.3, junto con los valores correspondientes a la energía de activación. Las curvas para metano y etano evidencian una region a las temperaturas más altas donde se presenta desviación del comportamiento lineal. A pesar de este hecho, se han utilizado todos los puntos para el cálculo de la energía de activación, teniendo en cuenta que los resultados así obtenidos servirán unicamente como criterio para la discusión cualitativa de las barreras energéticas difusionales.

Las barreras energéticas son bastante bajas para el caso de los adsorbatos menos voluminosos, metano y etano, con valores de 2.54 y $2.96 \mathrm{~kJ} / \mathrm{mol}$ respectivamente. A pesar de las diferencias apreciables entre los diámetros cinéticos de las moléculas la energía de activación es muy similar, lo que sugiere que el ta- 


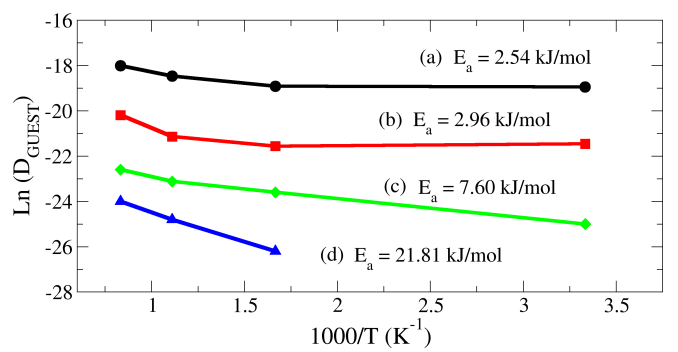

Figura 5.3: Diagramas de Arrhenius con los valores correspondientes a las energías de activación para (a) metano, (b) etano, (c) propeno y (d) propano adsorbidos en la zeolita Si-LTA.

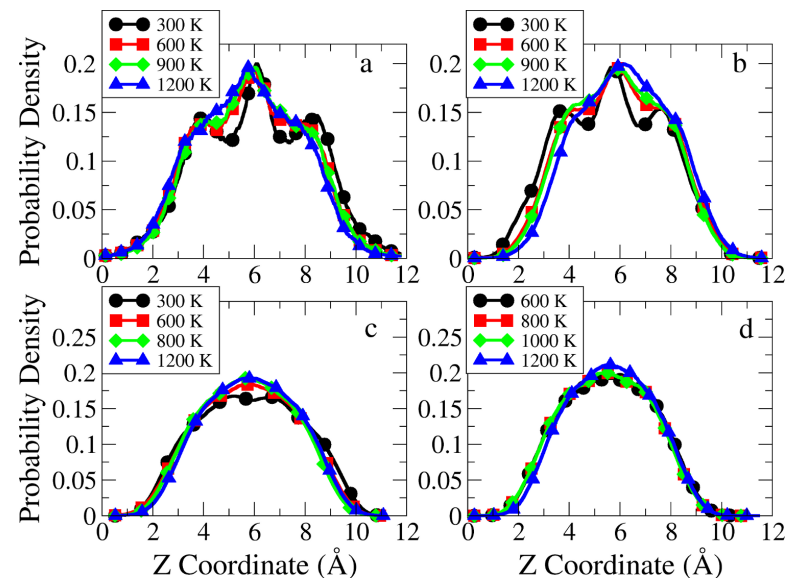

Figura 5.4: Distribución de probabilidad unidimensional a lo largo del eje z para el centro de masa de (a) metano, (b) etano, (c) propileno y (d) propano en la zeolita Si-LTA, y su dependencia con la temperatura. 
maño crítico de la ventana es lo suficientemente grande para permitir el paso de los dos adsorbatos sin imponer una barrera energética importante.

Las barreras energéticas que se perciben para el propeno y propano difieren sustancialmente, con valores de $7.6 \mathrm{~kJ} / \mathrm{mol}$ y $21.8 \mathrm{~kJ} / \mathrm{mol}$, respectivamente. Un aumento de aproximadamente tres veces la energía de activación para el propeno, en comparación con el metano y etano, evidencia la selectividad entrópica de las ventanas de conexión entre cavidades con respecto al diámetro cinético de los adsorbatos.

La energía de activación llega hasta $21.8 \mathrm{~kJ} / \mathrm{mol}$ para el propano, presentando un aumento de tres veces respecto al propeno. Este aumento en la barrera energética es consecuencia de la diferencia en tamaño entre los adsorbatos y además de la rigidez que el doble enlace le confiere a la molécula de propeno. El dramático incremento en las energías de activación confirma la potencialidad de este tipo de red porosa para afectar la dinámica de las especies adsorbidas con base en efectos puramente estéricos, especialmente notorios en el caso de las especies propano y propeno.

\subsubsection{Dinámica en la Zeolita Si-IHW}

La figura 5.5 recoje las curvas MSD para los adsorbatos C1-C3 en el rango de temperaturas 300-1200 K. En forma similar a la zeolita Si-LTA, se observa un aumento en el nivel de restricción al movimiento de los adsorbatos en función del tamaño molecular. El metano sigue un aumento constante en la pendiente del MSD con temperatura, aunque el espaciamiento entre MSDs se hace menor entre 900-1200 K. Este comportamiento sugiere que la excitación térmica del adsorbato afecta la dinámica hasta cierto punto, más allá del cual el efecto térmico deja de evidenciarse. Igual comportamiento sigue la molécula de etano, con una diferencia en la pendiente del MSD pequeña entre 900-1200 K.

La comparación de las escalas de los MSDs entre Si-IHW y Si-LTA sugiere una dinámica difusiva más restrictiva en la primera, la cual se confirma una vez calculados los coeficientes de difusión, que se presentan en la tabla 5.6. Es de notar la escala de la coordenada temporal en la figura 5.5 (a), la cual es dos veces más larga que su contraparte de la figura 5.2(a).

Las curvas de probabilidad de ocupación a lo largo de la coordenada Y, esquematizadas en la Figura 5.6, servirán para dilucidar las características difusionales en este sistema. Inicialmente, la figura 5.6(a) muestra la distribución de metano a lo largo del canal que posee una ventana 12-ring, con tres regiones de alta probabilida de ocupación claramente definidas. Esta distribución preferencial no se altera dramaticamente al aumentar la excitación térmica, aunque la distribución de probabilidades de ocupación tiende a desviarse hacia el centro del canal. La dis- 


\begin{tabular}{ccccc}
\hline & \multicolumn{4}{c}{ Coeficientes de Difusión $D_{s}\left(\frac{\mathrm{m}^{2}}{\mathrm{~s}}\right)$} \\
\cline { 2 - 5 } $\mathbf{T}(\mathbf{K})$ & $\mathbf{C H}_{\mathbf{4}}$ & $\mathbf{C}_{\mathbf{2}} \mathbf{H}_{\mathbf{6}}$ & $\mathbf{C}_{\mathbf{3}} \mathbf{H}_{\mathbf{6}}$ & $\mathbf{C}_{\mathbf{3}} \mathbf{H}_{\mathbf{8}}$ \\
\hline $\mathbf{3 0 0}$ & $9,4 \times 10^{-10}$ & $4,0 \times 10^{-11}$ & $8,3 \times 10^{-13}$ & \\
$\mathbf{6 0 0}$ & $3,9 \times 10^{-9}$ & $1,1 \times 10^{-10}$ & $8,2 \times 10^{-12}$ & $7,0 \times 10^{-14}$ \\
$\mathbf{9 0 0}$ & $6,5 \times 10^{-9}$ & $3,2 \times 10^{-10}$ & $1,1 \times 10^{-11}$ & $2,7 \times 10^{-12}$ \\
$\mathbf{1 2 0 0}$ & $7,7 \times 10^{-9}$ & $3,5 \times 10^{-10}$ & $5,6 \times 10^{-11}$ & $1,5 \times 10^{-11}$ \\
\hline
\end{tabular}

Tabla 5.6: Coeficientes de Difusión calculados para metano y etano en la zeolita ITQ-32 (SiIHW).
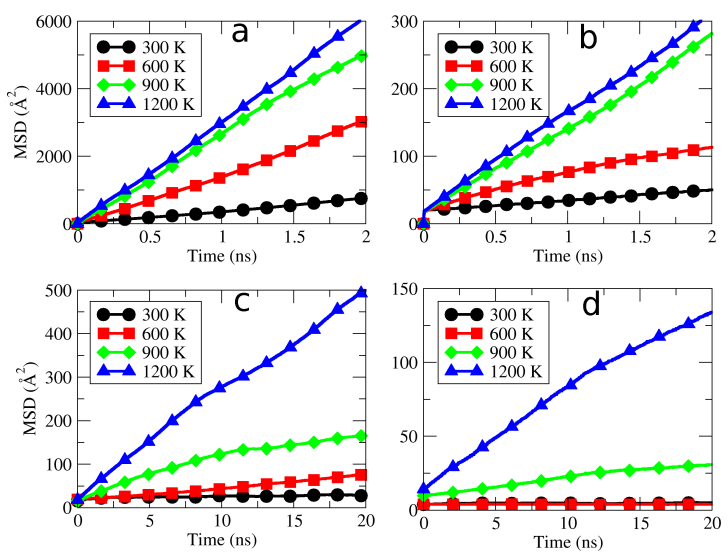

Figura 5.5: Perfiles MSD para (a) metano, (b) etano, (c) propeno y (d) propano adsorbido en la zeolita ITQ-32 (Si-IHW) a diferentes temperaturas.

tribución de las zonas preferenciales es casi idéntica para el etano, Figura 5.6(b), mostrando las tres regiones una dependencia similar respecto a la temperatura que para el caso del metano.

El mapa tridimensional de ocupación generado para la trayectoria de metano a alta temperatura se puede ver en la figura 5.7. Esta figura muestra las zonas de ubicación del centro de masa de la molécula de metano, coincidiendo las zonas de menor ocupación con la ubicación de las ventanas 8-ring, y las zonas preferenciales de adsorción ubicadas en el canal longitudinal formada por el anillo de 12 unidades de $\mathrm{SiO}_{4}$. Es evidente de la gráfica el camino bidimensional seguido por el adsorbato en esta estructura, lo que genera un cierto nivel de anisotropía en las características difusivas en el sistema.

La probabilidad de ocupación para las moléculas de propano y propeno muestran una movilidad más restringida para estas especies, con dos regiones preferenciales visibles a las temperaturas más bajas. El incremento de la temperatura 


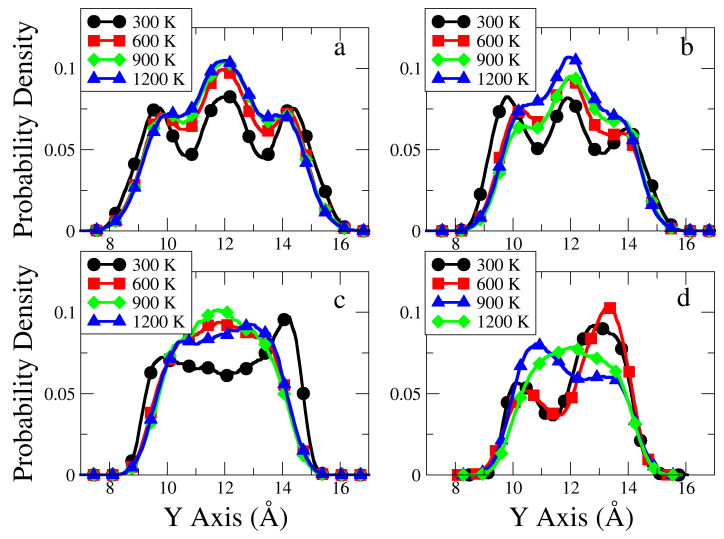

Figura 5.6: Distribución de probabilidad de ocupación unidimensional a lo largo del eje Y para el centro de masa de (a) metano, (b) etano, (c) propeno y (d) propano en la zeolita Si-IHW, y su dependencia con la temperatura.

se traduce en un confinamiento intra-poro menos restrictivo, permitiendo a las moléculas superar la barrera interna de la cavidad más frecuentemente, y por tanto generando una distribución de ocupación más amplia. En el caso particular del propano, el nivel de confinamiento intra-poro es mayor, y por tanto la distribución de posiciones en dos posiciones preferenciales se mantiene aún a $900 \mathrm{~K}$. La excitación cinética del propano se hace lo suficientemente alta a $1200 \mathrm{~K}$ para superar la barrera energética presente en la mitad del canal, dando como resultado una distribución única en el perfil de ocupación. Los coeficientes de difusión presentados en la tabla 5.6 reflejan numericamente el incremento de las restricciones a la movilidad de los adsorbatos, siendo el incrementeo significativamente mayor comparado con el caso previo de la zeolita Si-LTA.

La figura 5.8 muestra los valores calculados de energías de activación para los adsorbatos en la zeolita Si-IHW. Existe un muy buen comportamiento lineal en la dependencia de los coeficientes de difusión y la temperatura, de acuerdo al modelo de Arrhenius. Es de destacar la pequeña diferencia en las energías de activación para las moléculas de metano y etano en esta estructura, aunque la barrera energética es casi tres veces mayor en comparación con la zeolita SiLTA. Esta diferencia en energía de activación puede corresponder a dos factores topológicos principales, inicialmente la aparentemente pequeña diferencia en los tamaños de ventana, que llega a $0,6 \AA$, y la diferencia en la dimensionalidad de los canales. Las energías de activación calculadas para el propano y propeno son de $12.3 \mathrm{~kJ} / \mathrm{mol}$ y $54.1 \mathrm{~kJ} / \mathrm{mol}$, respectivamente. 


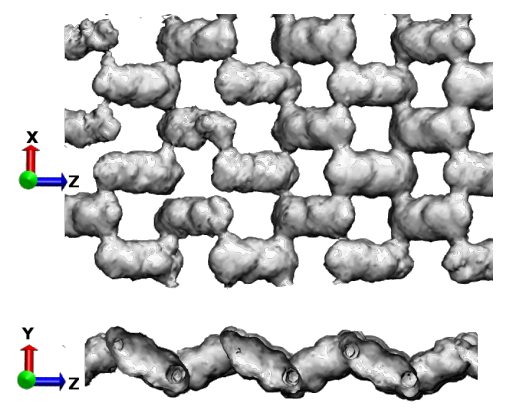

Figura 5.7: Mapa tridimensional de ocupación para el centro de masa del metano adsorbido en los canales de la zeolita Si-IHW.

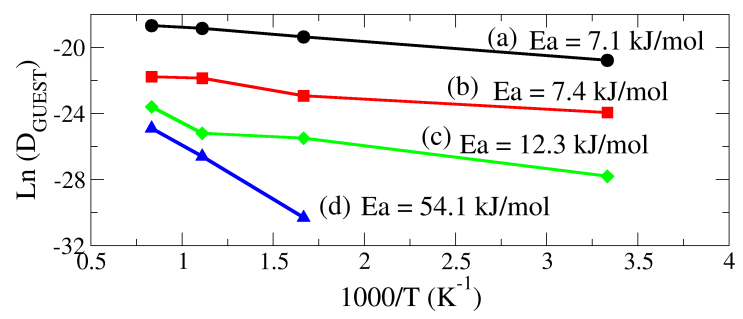

Figura 5.8: Diagramas de Arrhenius con los valores correspondientes a las energías de activación para (a) metano, (b) etano, (c) propeno y (d) propano adsorbidos en la zeolita Si-IHW. 


\begin{tabular}{ccccc}
\hline & \multicolumn{4}{c}{ Coeficiente de Difusión $D_{s}\left(\frac{\mathrm{m}^{2}}{\mathrm{~s}}\right)$} \\
\cline { 2 - 5 } $\mathbf{T}(\mathbf{K})$ & $\mathbf{C H}_{\mathbf{4}}$ & $\mathbf{C}_{\mathbf{2}} \mathbf{H}_{\mathbf{6}}$ & $\mathbf{C}_{\mathbf{3}} \mathbf{H}_{\mathbf{6}}$ & $\mathbf{C}_{\mathbf{3}} \mathbf{H}_{\mathbf{8}}$ \\
\hline $\mathbf{3 0 0}$ & $7,4 \times 10^{-9}$ & $4,3 \times 10^{-10}$ & $3,4 \times 10^{-12}$ & $1,0 \times 10^{-14}$ \\
$\mathbf{6 0 0}$ & $1,1 \times 10^{-8}$ & $1,2 \times 10^{-9}$ & $7,0 \times 10^{-11}$ & $5,7 \times 10^{-12}$ \\
$\mathbf{9 0 0}$ & $1,3 \times 10^{-8}$ & $1,8 \times 10^{-9}$ & $2,1 \times 10^{-10}$ & $5,9 \times 10^{-11}$ \\
$\mathbf{1 2 0 0}$ & $1,8 \times 10^{-8}$ & $2,3 \times 10^{-9}$ & $3,4 \times 10^{-10}$ & $1,6 \times 10^{-10}$ \\
\hline
\end{tabular}

Tabla 5.7: Coeficientes de Difusión calculados para metano, etano, propeno y propano en la zeolita ITQ-3 (Si-ITE).

\subsubsection{Dinámica en la Zeolita Si-ITE}

La figura 5.9 muestra las curvas de desplazamiento para metano, etano, propeno y propano adsorbidos en la zeolita ITQ-3, los correspondientes valores de los coeficientes de difusión se presentan en la tabla 5.7. El efecto de la dimensionalidad, que se cuantifica por el factor $N_{t}$ en la ecuación 5.1, refleja el número de grados traslacionales del adsorbato en el interior de la estructura cristalina. Así, esta estructura condiciona la movilidad de los adsorbatos en una única dimensión, obteniéndose coeficientes de difusión mayores que para la zeolita Si-LTA.

Las energías de activación calculadas para la fase adsorbida en esta estructura, y que se muestran en la figura 5.10, muestran un comportamiento para el modelo de Arrhenius altamente lineal, con energías de activación de Ea=2.6 kJ/mol y $\mathrm{Ea}=5.6 \mathrm{~kJ} / \mathrm{mol}$, para metano y etano respectivamente. Estos valores contrastan con las energías de activación calculadas para los mismos adsorbatos en Si-LTA y Si-IHW, en el sentido de que la energía impuesta por la barrera energética es dos veces mayor para etano en comparación con el metano, situación que no se presentaba en las estructuras mencionadas.

La energía de activación para la difusión de etano se correlaciona inversamente con el tamaño crítico de las ventanas en el orden Si-LTA $(4.1 \AA-2.96 \mathrm{~kJ} / \mathrm{mol})$ ¿Si- ITE $(3.8 \AA-5.6 \mathrm{~kJ} / \mathrm{mol})$ ¿Si-IHW $(3.5 \AA-7.4 \mathrm{~kJ} / \mathrm{mol})$, mostrando de forma sencilla la influencia de la topología de la red, en este caso el tamaño crítico de la barrera entre cavidades, con un evidente incremento en la energía necesaria para superar dicha barrera en función del tamaño del adsorbato.

La figura 5.11 muestra la superficie de densidad de ocupación para el centro de masa de la molécula de metano en la zeolita Si-ITE, donde resulta evidente la posición de las ventanas de 8-ring, formando cuellos de botella que restringen el movimiento del adsorbato entre celdas adyacentes.

La tabla 5.8 muestra los coeficientes de difusión experimentales y energías de activación para metano, etano y propano en la zeolita $5 \mathrm{~A}$ comparados con los resultados obtenidos en el presente trabajo. La zeolita 5A presenta un diámetro 

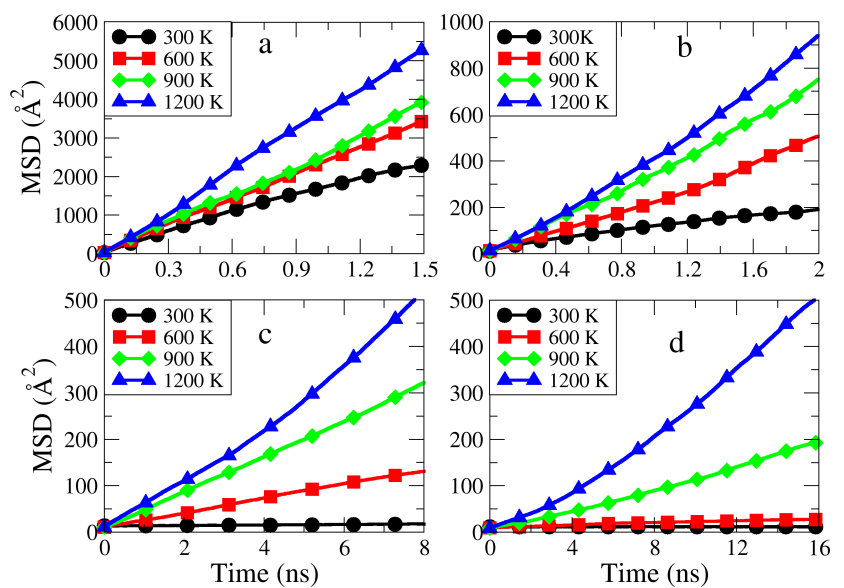

Figura 5.9: Perfiles MSD para (a) metano, (b) etano, (c) propeno y (d) propano adsorbido en la zeolita ITQ-3 (Si-ITE) a diferentes temperaturas.

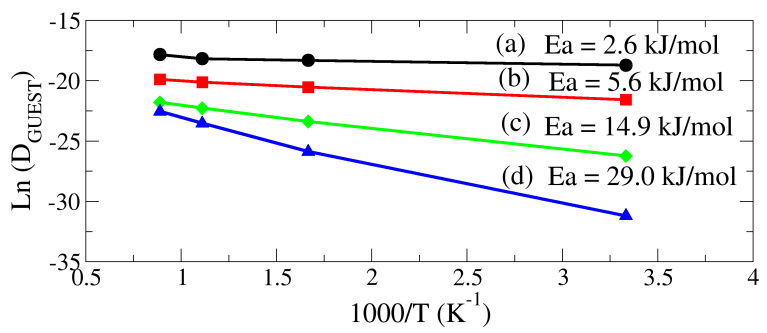

Figura 5.10: Diagramas de Arrhenius con los valores correspondientes a las energías de activación para (a) metano, (b) etano, (c) propeno y (d) propano adsorbidos en la zeolita ITQ-3 (Si-ITE) 


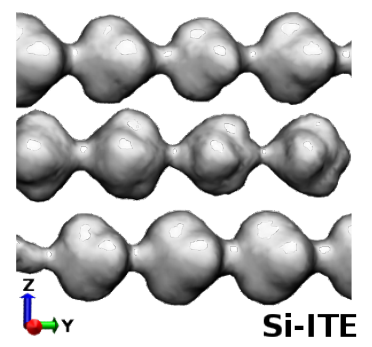

Figura 5.11: Mapa tridimensional de ocupación para el centro de masa del metano adsorbido en los canales de la zeolita Si-ITE.

crítico de ventana ligeramente mayor al de las dimensiones de su contraparte puramente silíceo, la zeolita Si-LTA. Los valores experimentales han sido tomados de la recopilación de Kärger y Ruthven. ${ }^{148}$ Las energías de activación obtenidas experimentalmente para la zeolita 5A son comparables a las calculadas, con la mayor discrepancia dada para propano como adsorbato. El alto nivel de coincidencia entre los datos experimentales y calculados generan un grado de confianza alto en los resultados calculados.

\subsubsection{Comparación de las características difusivas en Si-LTA, Si- IHW y Si-ITE}

La tabla 5.9 muestra comparativamente el tamaño de ventana crítico, el coeficiente de difusión y la energía de activación a $600 \mathrm{~K}$ para metano, etano y propeno. Hemos seleccionado la condición de temperatura de $600 \mathrm{~K}$ para nuestra comparación por el hecho de que la movilidad de los adsorbatos a esta temperatura permite una mayor certeza estadística en los coeficientes de difusión, y por consiguiente de la energías de activación calculadas. El diámetro crítico de la ventana de 8-ring sigue siendo un descriptor topológico importante para diferenciar las estructuras cristalinas, sin embargo, igualmente importante es la dimensionalidad de los canales.

Inicialmente es de resaltar la correlación existente entre el diámetro de ventana y la energía de activación. Para las tres estructuras el aumento de la energía de activación se correlaciona directamente con la disminución en el tamaño crítico de la ventana, sin embargo, los coeficientes de difusión para metano y etano no sigue la misma tendencia. En este caso, el hecho de que la zeolita Si-ITE presente canales unidimensionales hace que los valores obtenidos para $D_{s}$ sean mayores a los de las otras estructuras.

Los dos hidrocarburos C3 considerados necesitan de un análisis separado, 


\begin{tabular}{|c|c|c|c|c|c|c|c|c|c|}
\hline \multicolumn{10}{|c|}{ Coeficiente de Difusión $D_{s}\left(\frac{m^{2}}{s}\right)$ Ener } \\
\hline Zeolita & & $\mathrm{CH}_{4}$ & & & $\mathrm{C}_{2} \mathrm{H}_{6}$ & & & $\mathrm{C}_{3} \mathrm{H}_{8}$ & \\
\hline \multirow{4}{*}{$5 \mathrm{~A}$} & & $D_{s}$ & $A E$ & & $D_{s}$ & $A E$ & & $D_{s}$ & $A E$ \\
\hline & Neut. Diff. & $6,0 \times 10^{-10}$ & & ZLC & $3,0 \times 10^{-11}$ & 6,7 & NMR(PFG) & $2,0 \times 10^{-13}$ & \\
\hline & $\mathrm{NMR}(\mathrm{PFG})$ & $\sim 10^{-9}$ & 4 & NMR(PFG) & $6,0 \times 10^{-11}$ & 6,0 & Grav. Uptake & $2,0 \times 10^{-13}$ & 14,5 \\
\hline & & & & & & & Freq. Resp. & $2,0 \times 10^{-13}$ & \\
\hline Si-LTA & $300 \mathrm{~K}$ & $5,9 \times 10^{-9}$ & 2,5 & $300 \mathrm{~K}$ & $4,8 \times 10^{-10}$ & 3,0 & $600 \mathrm{~K}$ & $4,0 \times 10^{-12}$ & 21,8 \\
\hline Si-IHW & $300 \mathrm{~K}$ & $9,4 \times 10^{-10}$ & 7,1 & $300 \mathrm{~K}$ & $4,0 \times 10^{-11}$ & 7,4 & $600 \mathrm{~K}$ & $7,0 \times 10^{-14}$ & 54,1 \\
\hline Si-ITE & $300 \mathrm{~K}$ & $7,4 \times 10^{-9}$ & 2,6 & $300 \mathrm{~K}$ & $4,3 \times 10^{-10}$ & 5,6 & $300 \mathrm{~K}$ & $1,0 \times 10^{-14}$ & 29,0 \\
\hline
\end{tabular}

Tabla 5.8: Coeficientes de difusion experimentales para la zeolita 5A comparados con los valores calculados para las zeolitas Si-LTA, SI-IHW y Si-ITE.

porque las tendencias entre diámetros críticos de ventana y movilidad no son tan evidentes como en el caso de los hidrocarburos mencionados anteriormente. Para este caso, la tabla 5.10 relaciona los diámetros críticos de las ventanas, coeficientes de difusión y energías de activación.

El propeno muestra un incremento en la energía de activación correlacionada con el diámetro de ventana, con el valor más bajo para la zeolita Si-LTA, la cual también presenta el mayor diámetro crítico, y valores dos veces más grandes para el Si-ITE y Si-IHW. Para estas dos últimas estructuras la EA se incrementa por un factor de $\simeq 2$, mientras que los coeficientes de activación siguen la tendencia $D_{L T A} \simeq D_{I T Q}>D_{I H W}$.

la molécula de propano son un poco menos evidentes, ya que existe una discontinuidad muy notoria en el valor del coeficiente de difusión en la zeolita SiITE, y que nuevamente, es producto de la topología de la red, antes que del diámetro crítico de la ventana.

En el caso de la estructura Si-IHW, con canales bidimensionales que se extienden a lo largo de las coordenadas $\mathrm{X}$ y $\mathrm{Z}$ conectadas a traves de planos perpendiculares definidos por las ventanas de 8 tetraedros, existe un nivel de tortuosidad que incrementa el requerimiento energético del adsorbato para superar la barrera estérica, disminuyendo en similar proporción las oportunidades ejecutar saltos exitosos a posiciones vacantes en celdas vecinas.

\subsection{Conclusiones}

La técnica de la dinámica molecular ha sido utilizada para el estudio de la movilidad de hidrocarburos de bajo peso molecular, C1-C3, adsorbidos en tres estructuras zeolíticas puramente silíceas ITQ-29(Si-LTA), ITQ-32(Si-IHW) y ITQ- 


\begin{tabular}{cc|ccc}
\hline \multirow{2}{*}{ Estructura } & $\boldsymbol{\emptyset}_{\text {Critico }}(\mathbf{\AA})$ & $\mathbf{C H}_{\mathbf{4}}$ & $\mathbf{C}_{\mathbf{2}} \mathbf{H}_{\mathbf{6}}$ & $\mathbf{C}_{\mathbf{3}} \mathbf{H}_{\mathbf{6}}$ \\
\cline { 3 - 5 } & 4,1 & $6,1 \times 10^{-9}(2,5)$ & $4,3 \times 10^{-10}(2,9)$ & $5,6 \times 10^{-11}(7,6)$ \\
Si-LTA & 3,8 & $1,1 \times 10^{-8}(2,6)$ & $1,2 \times 10^{-9}(5,6)$ & $7,0 \times 10^{-11}(14,9)$ \\
Si-ITE & 3,5 & $3,9 \times 10^{-9}(7,1)$ & $1,1 \times 10^{-10}(7,4)$ & $8,2 \times 10^{-12}(12,3)$ \\
Si-IHW & & &
\end{tabular}

Tabla 5.9: Comparación entre los diámetros críticos de las ventanas de 8-ring (distancias $O-O$ ), coeficientes de difusión y energías de activación (EA) para metano, etano y propeno en zeolitas a $600 \mathrm{~K}$.

\begin{tabular}{cc|cc}
\hline \multicolumn{2}{c}{} & \multicolumn{2}{c}{ Coeficiente de Dif. $D_{s}\left(\frac{m^{2}}{s}\right) A E\left(\frac{k J}{m o l}\right)$} \\
\cline { 3 - 4 } Estructura & $\boldsymbol{\emptyset}_{\text {Critico }}(\AA)$ & $\mathbf{C}_{\mathbf{3}} \mathbf{H}_{\mathbf{6}}$ & $\mathbf{C}_{\mathbf{3}} \mathbf{H}_{\mathbf{8}}$ \\
\hline Si-LTA & 4,1 & $1,5 \times 10^{-10}(7,6)$ & $3,8 \times 10^{-11}(21,8)$ \\
Si-ITE & 3,8 & $3,4 \times 10^{-10}(14,9)$ & $1,6 \times 10^{-10}(29,9)$ \\
Si-IHW & 3,5 & $5,6 \times 10^{-11}(12,3)$ & $1,5 \times 10^{-11}(54,1)$ \\
\hline
\end{tabular}

Tabla 5.10: Comparación entre los diámetros críticos de las ventanas de 8-ring (distancias $O-O$ ), coeficientes de difusión y energías de activación (EA) para propano y propeno en zeolitas a 1200 K.

3(Si-ITE), todas ellas con una ventana característica de 8 unidades tetraédricas que comunica los poros de las diferentes estructuras y representa la barrera limitante para el proceso de difusión.

En general los coeficientes de difusión muestran una correlación inversa entre el diámetro cinético de los adsorbatos y el diámetro crítico de las ventanas, confirmando así medidas experimentales que definían la apertura de las ventanas como la barrera difusiva. El movimiento traslacional de largo alcance es influenciado también por las características topológicas de la red cristalina, siendo la dimensionalidad de la red la que ejerce la principal influencia en los coeficientes de difusión, a través del factor $\mathrm{N}$ en la ecuación 5.1.

La influencia directa del tamaño de la ventana es apreciada claramente comparando las constantes difusionales de los adsorbatos de menor tamaño, metano y etano, lo que resulta en mayores constantes difusivas en la zeolita Si-LTA, la cual posee el diámetro crítico de ventana más alto, mientras que los valores de difusividad son menores para la zeolita Si-IHW, con el menor diámetro crítico de ventana. Las energías de activación, muestran una tendencia similar, con barreras energéticas mayores adscritas a los ventanas de menor apertura.

La relación entre energías de activación, definida como

$$
\delta E A^{Z E O}=E A^{\text {Propeno }} / E A^{\text {Propano }}
$$


es igual a $\delta E A^{L T A}=0,35, \delta E A^{I T E}=0,5$ y $\delta E A^{I H W}=0,23$.

Las selectividades difusionales, calculadas como

$$
S(D)^{Z E O}=D^{\text {Propeno }} / D^{\text {Propano }}
$$

producen valores de $S(D)^{I T E}=14,0, S(D)^{I H W}=117$ y $S(D)^{I T E}=12 \mathrm{a}$ $600 \mathrm{~K}$.

A partir de estos valores, concluimos que las selectividades difusionales más altas estarán asignadas a la zeolita ITQ-32, mientras que la estructura de la zeolita ITQ-29 no permitiría llevar a cabo un proceso de separación extremadamente selectivo. Sin embargo, los bajos valores de los coeficientes de difusión en la zeolita ITQ-32 serían poco atractivos para llevar a cabo el proceso, ya que se requeriría de un tiempo de contacto con la fase fluida posiblemente prohibitivo a nivel industrial. 


\title{
Capítulo 6
}

\section{Rol de la Superficie en el Proceso de Adsorción de Metano en la Zeolita Si-LTA}

\begin{abstract}
Con ayuda de la técnica computacional de la Dinámica Molecular se ha simulado el proceso de adsorción superficial de una fase gaseosa de metano en un modelo de zeolita Si-LTA con grupos silanol explícitos. Usando herramientas de cálculo tales como mapas tridimensionales de ocupación, perfiles de densidad de ocupación y de energía libre se ha logrado estudiar el proceso de adsorción a nivel atomístico, pudiendo llegar a detallarse las zonas preferenciales de interacción superficial y su impacto en el proceso adsortivo en estado transitorio y de equilibrio. La influencia de la concentración sobre el proceso transitorio de adsorción se ha monitorizado siguiendo la variación del número de moléculas que efectivamente cruzan la superficie y llegan al interior del cristal, esta información permite caracterizar la permeabilidad superficial del sistema. Se discuten las implicaciones de la permeabilidad superficial a la luz de resultados experimentales obtenidos recientemente.
\end{abstract}

En el pasado reciente se ha llevado a cabo un esfuerzo de investigación considerable en el estudio de los fenómenos básicos en la difusión y adsorción de especies confinadas en materiales nanoporosos. En especial, el excelente trabajo desarrollado por Jörg Kärger y su grupo de investigación ha permitido que la comunidad científica retome el estudio de los fundamentos de los procesos de difusión. ${ }^{148,171,172}$ Con el desarrollo de nuevas técnicas y el perfeccionamiento 
Rol de la Superficie en el Proceso de Adsorción de Metano en la Zeolita

de las ya existentes, tales como el "pulse field gradient NMR" (PFG-NMR) y la microscopía de alta resolución por transmisión de electrones (HR-TEM), ${ }^{173}$ se ha podido llegar a un nivel de resolución en el que es posible estudiar el movimiento molecular en escalas de tamaño lo suficientemente pequeñas como para detallar el movimiento puramente intracristalino de las fases adsorbidad y por supuesto para la caracterización de resistencias al transporte de masa debida a barreras de superficie y a la influencia de los defectos en los adsorbentes. ${ }^{172,174-177}$ A pesar de lo anterior, la investigación en fenómenos de transferencia de masa se han centrado en el estudio del transporte de la fase adsorbida en los sólidos cristalinos, mientras que la influencia de las barreras superficiales y de los defectos estructurales en los procesos de sorción y de "release", solo han empezado a estudiarse muy recientemente. ${ }^{175,177-184}$

Una de las principales barreras para el estudio de fenómenos de superficie es la asociada con los avance en las técnicas experimentales de caracterización de sólidos. Solo recientemente se han reportado estudios sobre la estructura superficial de sólidos como la zeolita silicalita-1, utilizando la técnica de HR-TEM, ${ }^{185}$ o del uso de la técnica de microscopía de fuerza atómica (AFM) para la identificación de los detalles estructurales de las zeolitas mordenita (MOR), HEU y LTA. ${ }^{186,187}$ Estos avances experimentales proveen la información necesaria de la que se alimentan las técnicas computacionales para la definición de modelos que reflejen las posibles características de las superficies en materiales de interés.

En esta linea, existen algunos trabajos computacionales que integran en la descripción del campo de fuerza del sistema la presencia de grupos $\mathrm{SiOH}$ superficiales. ${ }^{115,180,182,184,188,189}$ En el presente trabajo hemos utilizado la técnica de la dinámica molecular para estudiar el proceso de adsorción de metano en la zeolita Si-LTA en su etapas transitoria y estable. Nuestro modelo integra en la descripción del campo de fuerza la presencia de grupos silanol explícitamente.

La zeolita ITQ-29 (Si-LTA) ha sido la escogida como modelo por su importancia en el campo industrial, además de por ser una zeolita modelo para estudios tanto experimentales como computacionales. Recientemente, Slater et al. ${ }^{180}$ han publicado un trabajo en el que se caracteriza la estructura superficial de la zeolita Si-LTA a través de cálculos de energías relativas utilizando la técnica de la dinámica de redes. Este estudio ha sido complementario al trabajo experimental de Wakihara et. al., ${ }^{187}$ en el cual se usa la técnica HR-TEM para resolver la estructura superficial de la zeolita Si-LTA, trabajo que sin embargo no arroja resultados totalmente conclusivos.

En este capítulo retomamos los resultados del trabajo de Slater et al. ${ }^{180}$ para le definición del campo de fuerza de la zeolita ITQ-29 en el proceso de adsorción de metano en dicha estructura. Utilizando las trayectorias obtenidas a través de la técnica de la dinámica molecular se describe el proceso transitorio de adsorción 
de metano, a diferentes concentraciones, en un cristal modelo de zeolita ITQ-29 hasta alcanzar el estado de equilibrio. De esta manera, y tal como se describe más adelante, se puede relacionar el flujo de partículas que efectivamente logran cruzar la superficie y ser adsorbidas por el material con las barreras generadas por la discontinuidad entre la fase gaseosa y la superficie del sólido poroso.

\subsection{Modelo del Sistema}

Tal como se mencionó anteriormente, la selección de la zeolita ITQ-29 se debe a su amplia utilización industrial y al conjunto de datos experimentales y computacionales existentes. La zeolita ITQ-29 puede describirse como la contraparte puramente silícea de los materiales de red LTA, y puede definirse estructuralmente como una red de cavidades esféricas (celdas- $\alpha$ ), interconectadas tridimensionalmente, de longitud aproximada a $11.4 \AA$ conectadas por seis ventanas de tamaño pequeño (8-ring) con diámetro efectivo de $4.1 \AA$.

Alternativamente, la estructura LTA puede describirse como una red formada por celdas de sodalita (celdas- $\beta$ ) conectadas entre sí por sus caras cuadradas planas, formando así anillos dobles de dimensión 4-ring. Esta estructura ofrece la posibilidad de difusión isotrópica en los tres ejes cristalográficos.

La figura 6.1 muestra las posibles configuraciones terminales de la superficie de la zeolita ITQ-29, terminación 4-T simple (S4R), terminación 4-T doble (D4R-e), y doble anillo 4-T terminal (D4R-f) para la superficie $\{100\}$, siguiendo la terminología propuesta por Slater et al. ${ }^{184}$ Los cálculos energéticos de dinámica de redes muestran que la configuración terminal S4R es más estable que las configuraciones terminadas en dobles anillos, en oposición a los resultados experimentales, ${ }^{186,187}$ con las discrepancias siendo adscritas a la posibilidad de problemas en la estabilidad cinética o incluso a la presencia de iones estabilizadores en la solución de síntesis.

A pesar del cuestionamiento de los resultados experimentales citados anteriormente por Slater, y en base a la mínima diferencia energética encontrada en sus cálculos, se ha tomado la terminación D4R-e como la configuración superficial "real" más posible, selección reforzada además por estudios experimentales usando la técnica de AFM. ${ }^{186}$

Las celdas de simulación se han generado haciendo un escalamiento adecuado de la celda unidad, seguida por el "cleavage" y completado de las terminaciones $\mathrm{SiO}_{3 / 2}^{+}$con unidades $\mathrm{OH}^{-}$. La tabla 6.1 muestra los detalles de la celda unidad y la celda de simulación modelada. 
Rol de la Superficie en el Proceso de Adsorción de Metano en la Zeolita

\begin{tabular}{lcccc}
\hline Zeolita & $\mathbf{a}[\mathbf{\AA}]$ & $\mathbf{b}[\mathbf{\AA}]$ & $\mathbf{c}[\AA \mathbf{\Omega}]$ & $\alpha=\beta=\gamma[\mathbf{G r a d}]$ \\
\hline & 11.92 & 11.92 & 11.92 & 90.0 \\
\cline { 2 - 5 } (ITQ-29) LTA $^{\mathbf{1 2}}$ & Celda SiO $_{4 / 2}$ & Celda de Sim. + Superficie \\
& $2 \times 2 \times 4-1152$ atoms & $2 \times 2 \times 4-1008$ atoms & \\
& & &
\end{tabular}

Tabla 6.1: Parámetros de red de la zeolita ITQ-29 y detalles de la celda de simulación
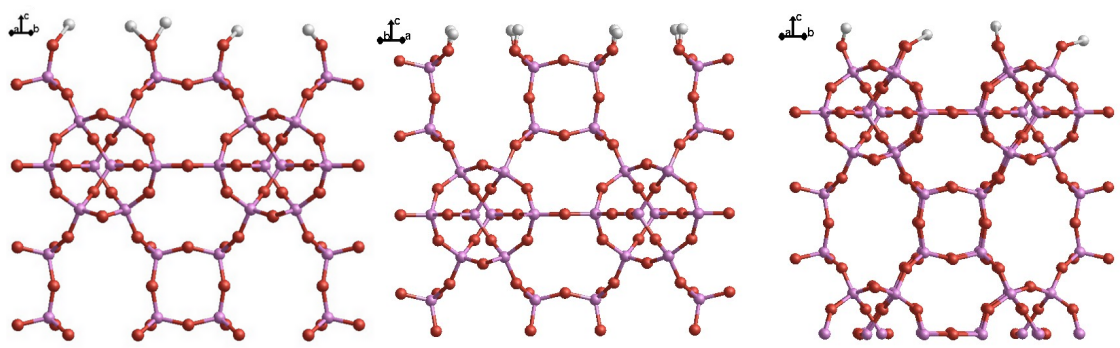

Figura 6.1: Superficies $\{100\}$ hidroxiladas posibles para la estructura Si-LTA: izquierda $S 4 R$, centro D4R-edge, y derecha D4R-f. Los colores corresponden a tipos de átomos: Rojo (silicio), violeta (oxígeno) y blanco (hidrógeno).

\subsection{Detalles de la Metodología de Cálculo}

La técnica utilizada en este trabajo es la dinámica molecular, descrita en capítulos anteriores. Sin embargo, la inclusión de la superficie en el modelo del sólido cristalino conlleva una modificación de los modelos descritos en capítulos anteriores, ya que la superficie de energía potencial es aumentada integrando las cargas y detalles de interacciones entre los átomos de la superficie y los átomos de la red interna así como de las interacciones adsorbato superficie.

El potencial de interacción toma los trés términos de interacción normales, interno de la zeolita $V^{Z e o}$, interno del adsorbato $V^{M}$ y de interacción zeolita adsorbato $V^{Z e o-M / M-M}$, de acuerdo al modelo:

$$
V^{T o t a l}=V^{Z e o}+V^{M}+V^{Z e o-M / M-M}
$$

El término $V^{M}$, representa las interacciones internas del metano, y comprende términos de enlace entre dos partículas más las interacciones electrostáticas del caso. Los parámetros para esta parte del potencial se dan en la referencia original de Oie et al. ${ }^{158} \mathrm{El}$ factor $V^{Z e o-M / M-M}$ se ha modelado usando interacciones de tipo Lennard-Jones (LJ 12-6).

El término más complejo es, por supuesto, el que describe la red cristalina 
y la superficie con grupos silanol. Para este caso, inicialmente se usa el modelo de Sanders et al. ${ }^{69}$ para describir el interior de la zeolita, complementado para la superficie con el modelo de Baram-Parker, ${ }^{190}$ en forma similar a la definida en el trabajo de Gren et al. ${ }^{180}$

Las dos partes del potencial están basadas en el modelo core-shell, el cual requiere para su aplicación en dinámica molecular el uso de un paso de integración del orden de $0.02 \mathrm{fs}$, lo suficientemente bajo para evitar cualquier posibilidad de transferencia energética intermolecular debida a colisiones, y para un apropiado modelamiento de las características vibracionales de los grupos $\mathrm{OH}^{-}$superficiales.

Sin embargo, un delta de tiempo muy pequeño conlleva la necesidad de extender el tiempo de simulación más allá de límites de eficiencia convenientes. Por lo tanto, inicialmente se evalúa el impacto de la flexibilidad de la red en la dinámica del sistema. De antemano sabemos que la influencia de la flexibilidad de la red para el sistema específico Si-LTA/Metano no es crítico, ${ }^{191,192}$ ya que el tamaño de la ventana de 8-T es lo suficientemente amplio para no impedir el paso del metano de forma importante.

Inicialmente evaluamos los perfiles de ocupación que se obtienen con la red flexible y la red fija, obteniendo perfiles de ocupación virtualmente idénticos para las dos condiciones, por lo que se decide mantener la red flexible para los cálculos posteriores. El modelamiento de la red fija permite llevar a cabo simulaciones que se extienden hasta por $4 x 10^{6}$ pasos, equivalente a $4 \mathrm{~ns}$, con un costo computacional adecuado.

El procedimiento para llevar a cabo las simulaciones se detalla a continuación. El espacio entre las capas de zeolita (ver detalles de la celda de simulación en la figura 6.2) es inicialmente llenado con un número determinado de moléculas de metano, despues de lo cual se permite la relajación hasta alcanzar el equilibrio. Se monitorea el número de moléculas que pasan a través de la superficie y alcanzan el interior de la celda en función del tiempo (número de pasos de simulación) hasta que se alcanza el equilibrio, condición que se asimila a un flujo neto igual a cero través de la superficie.

El algoritmo de Velocity-Verlet se utiliza para la integración de las ecuaciones de movimiento de Newton, con pasos de integración igual a $1 \times 10^{-15}$ s o 1 fs. El número de moléculas en el espacio interlaminar varía entre 20-200, con incrementos para cada sistema de 20 moléculas. El muestreo de microestados de lleva a cabo en el colectivo microcanónico (NVT), a una temperatura de $300 \mathrm{~K}$ obtenido utilizando el termostato de Berendsen, con constante de tiempo de 4 ps. ${ }^{68,169}$ 


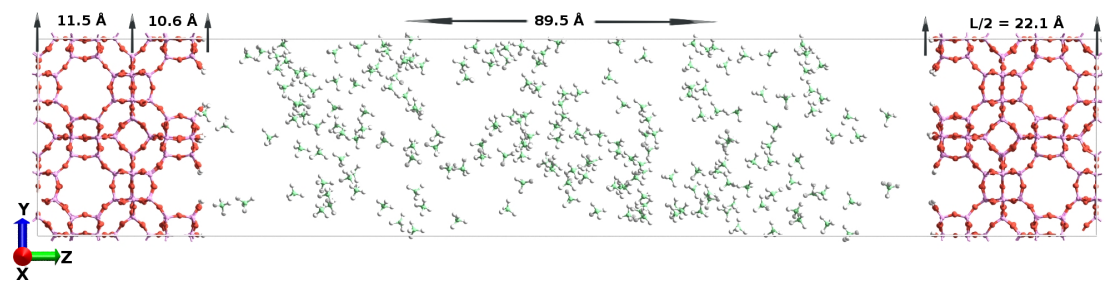

Figura 6.2: Imagen de la celda de simulación con un contenido inicial de 180 moléculas de metano en el espacio interlaminar. Condiciones períodicas de frontera se aplican en los tres ejes de la celda. La celda simulada bajo estas condiciones representa láminas de dimensiones infinitas en el plano XY, y de longitud igual a 4 celdas unidad a lo largo del eje $Z$, lo que forma una membrana con láminas separadas por un espacio de $89.5 \AA ̊$ de longitud.

\subsection{Resistencia Superficial al Transporte de Masa}

El análisis del proceso de adsorción se inicia definiendo como variable el número de moléculas $(N(t))$ en la fase gaseosa que cruzan la superficie, definida por el plano con superficie $(A)$ formado por los átomos externos del sólido cristalino, y que efectivamente logran alcanzar el interior del cristal por unidad de tiempo (ver figura 6.3).

El proceso se considera en equilibrio una vez se llegue a un balance en el número de moléculas en cualquiera de las dos secciones del sistema, lo que representaría un estado de flujo neto igual a cero.

En la interfase, $z=22,1 \AA$ en la figura 6.2, se define el flujo de moléculas $j(t)$ como la variación en el número de moléculas que atraviesan la superficie por unidad de tiempo, en función del gradiente de concentración en $\mathrm{Z}$, de acuerdo a:

$$
j(t)=\alpha_{s}\left(C(t)_{e q}-C(t)\right)=\frac{1}{2 A} \frac{d N(t)}{d t}
$$

donde $C(t)=\frac{N(t)}{A L}$ define la concentración de moléculas en la superficie, $C(t)_{e q}$ la concentración de moléculas que se encuentra en equilibrio con la fase gaseosa y $\alpha_{s}$ (ver ecuación 6.3) representa la permeabilidad superficial, parámetro que abarcará la información relativa a las resistencias superficiales a la transferencia de masa. El factor 2 en la ecuación 6.2 resulta del hecho de que existe un flujo de moléculas en las dos caras de la zeolita, lo que supone un area de cruce igual a dos veces el área superficial de la celda de simulación.

$$
\alpha_{s}=\frac{L}{2\left(N(t)_{e q}-N(t)\right)} \frac{d N(t)}{d t}
$$




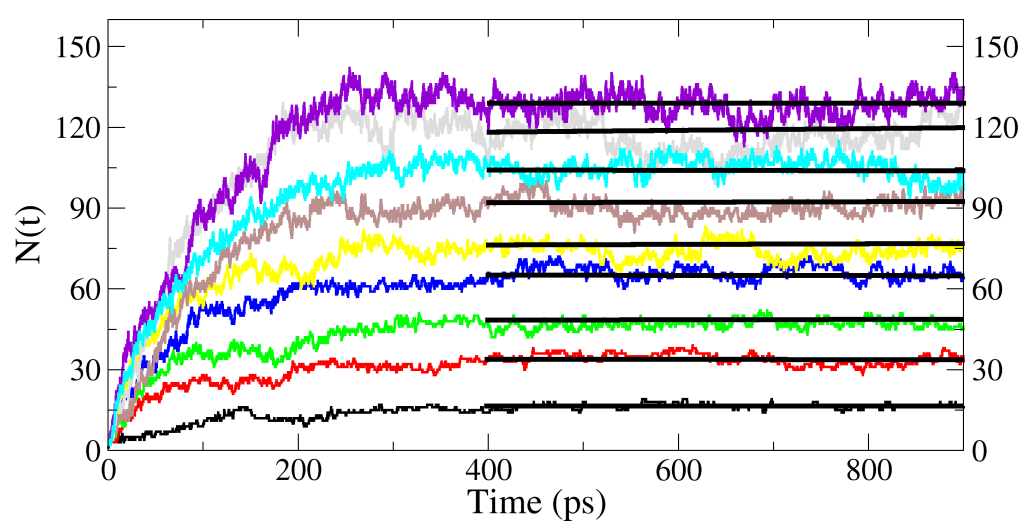

Figura 6.3: Variación en el espacio temporal del número de moléculas $N(t)$ a través de la superficie $A$ del sólido cristalino. Las diferentes curvas corresponden a sistemas con número inicial de moléculas en el espacio interlaminar variando entre 20-200. El estado de equilibrio, y el número de moléculas en el interior de la zeolita en equilibrio con la concentración en la fase gaseosa, se representa por líneas rectas horizontales para tiempos de simulación de $>400 p s$.

De las trayectorias obtenidas por las simulaciones de DM, se obtiene inmediatamente la dependencia temporal del número de moléculas que atraviezan la superficie, dado por $N(t)$, la variación del número de moléculas en la fase gaseosa y por lo tanto el número de moléculas en equilibrio con la fase gaseosa, $N(t)_{e q}$.

\subsection{Resultados y Discusión}

La consistencia de los resultados extraídos de las simulaciones es puesta a prueba en comparación con isotermas de adsorción publicados para el sistema Si-LTA/Metano. ${ }^{58,193}$ La cantidad de moléculas de metano calculadas en las simulaciones (ver figura 6.4) se compara de forma muy cercana con las isotermas correspondientes calculadas a condiciones similares.

En el sistema con 200 moléculas, hemos calculado una cantidad de moléculas adsorbidas igual a 7 por celda- $\alpha$. El valor de saturación para metano en la zeolita Si-LTA es de cerca de 16 moléculas por celda- $\alpha$, localizando las condiciones de concentración en nuestros sistemas en una región de presión (concentración) entre baja-intermedia en las isotermas de adsorción. 


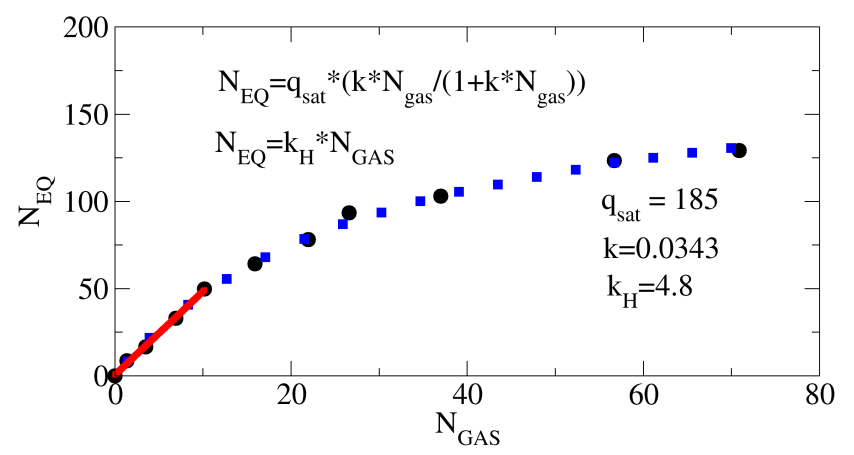

Figura 6.4: Isoterma que representa el número de moléculas adsorbidas en el sólido cristalino que están en equilibrio con una concentración dada de metano en la fase gaseosa. La línea recta de color rojo corresponde a la región de Henry, donde la dependencia entre las concentraciones sigue una relación lineal. La linea punteada representa el ajuste a una isoterma de Langmuir. Los parámetros de cada ajuste se dan en la figura

\subsubsection{Zonas de Probabilidad de Ocupación y Energía Libre}

La figura 6.5 muestra los perfiles bidimensionales de probabilidad de ocupación del adsorbato a lo largo del eje de adsorción, eje $\mathrm{Z}$ en la celda de simulación, obtenida para las trayectorias en las condiciones descritas anteriormente. Adicionalmente, la figura 6.5 muestra los perfiles de energía libre, calculados a partir de densidad ocupacional.

La figura identifica tres regiones diferentes: el espacio interlaminar correspondiente a $z>22,1 \AA$, una región intermedia comprendida por las celdas- $\alpha$ parcialmente clivadas y expuestas a la región exterior entre $11,5<z<22,1 \AA$, y la zona interior localizada en el rango $0<z<11,5 \AA$ que integra las celdas- $\alpha$ completas limitadas en $z=0$ por las ventanas 8 -ring.

La región ubicada a la derecha de la superficie de la zeolita, $z>22,1 \AA$ en la figura 6.5, muestra la existencia de una zona preferencial para las moléculas de metano, representada por el pico de concentración justo en la superficie. Esta zona sugiere la existencia de regiones preferenciales de adsorción superficial, pudiendo ser racionalizadas como consecuencia de las interacciones atractivas de van der Waals que surgen entre el adsorbato y los átomos de oxígeno parcialmente expuestos de los grupos silanol.

El comportamiento atractivo de estas zonas es confirmado mirando las proyeccioes bidimensionales capturadas justo sobre la superficie de los grupos silanol, en la región localizada entre los grupos $\mathrm{OH}$ mostrados en rojo en la parte 

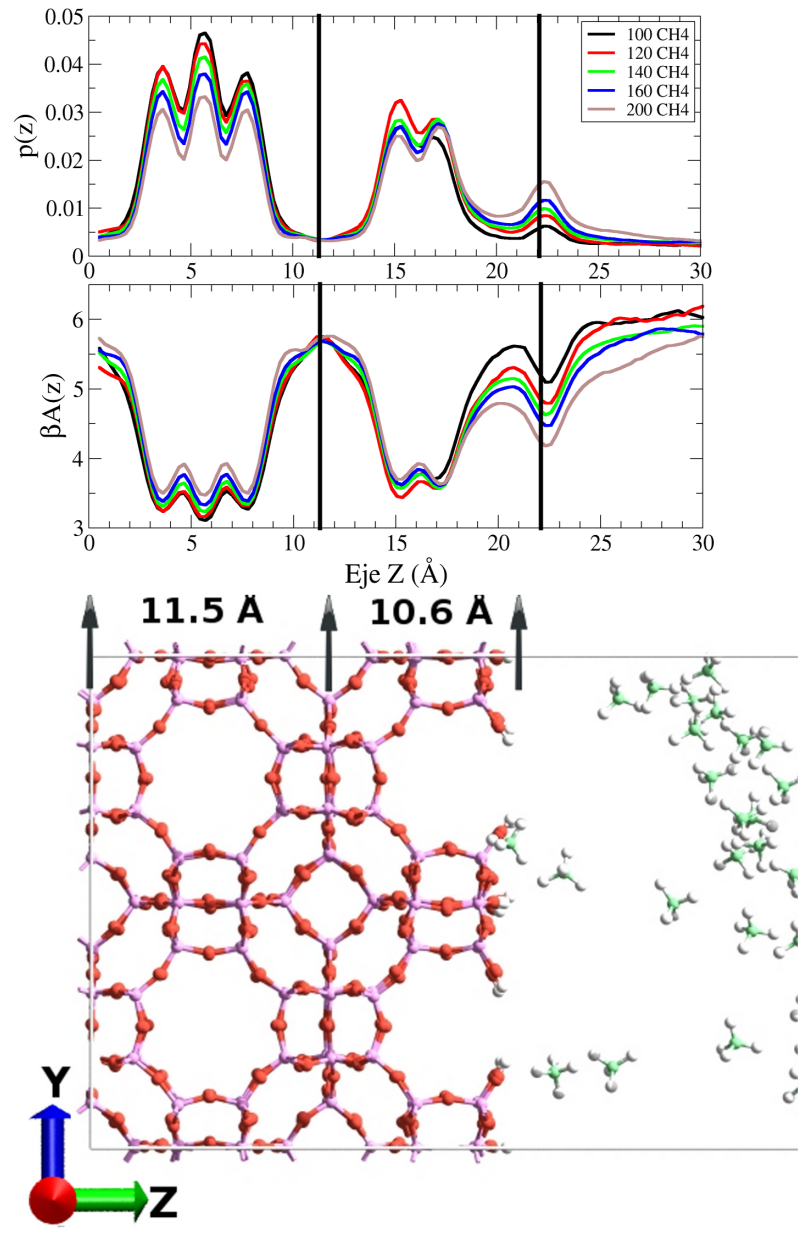

Figura 6.5: Densidad de ocupación P(z) (arriba) y perfiles de energía libre (mitad) experimentada por la fase adsorbida y calculados a partir de histogramas con la información de las trayectorias de DM a diferentes condiciones de concentración. Las lineas verticales muestran las regiones correspondientes a la ubicación de las ventanas de 8-ring y a la superficie formada por los grupos silanol localizados en la superficie. 


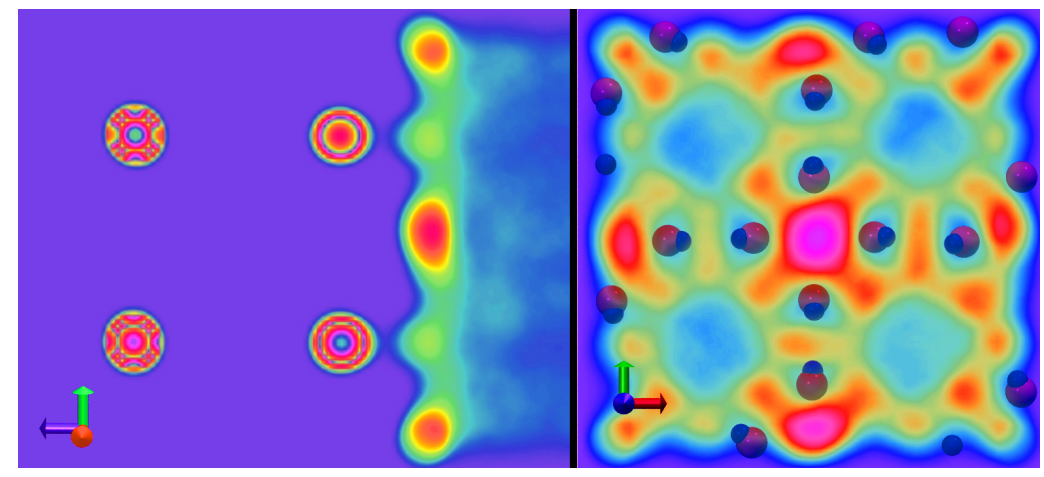

Figura 6.6: Proyección bidimensional del mapa de ocupación tridimensional de metano a lo largo del eje Z, plano perpendicular ZY en la mitad de la celda de simulación (izquierda). Proyección en el plano $X Y$ sobre los grupos silanol (derecha), a partir de los volúmenes tridimensionales de ocupación, generados a partir de una trayectoria de DM. Las zonas de ocupación preferenciales se muestran en rojo, mientras que las zonas de ocupación nula están dadas por las zonas azul/violeta. La posición de los grupos silanol en la superficie se muestran como esferas de van der Waals en rojo (oxígeno) y azul (hidrógeno).

derecha de la figura 6.6.

La segunda característica sobresaliente de la región superficial es su dependencia de la concentración, la cual es evidente en la figura 6.5, con las zonas de densidad poblacional aumentando en razón directa del número de la concentración de metano en el sistema.

La región intermedia del sistema, que comprende las celdas- $\alpha$ parcialmente clivadas, es cualitativamente diferente de la superficie y la parte interior del cristal. Solo dos picos con densidad poblacional alta son visibles en esta región, reflejando la influencia de la topología de la red en cuanto al número de sitios preferenciales para la adsorción.

La baja densidad de ocupación, pico derecho de la zona intermedia $11,5<$ $z<22,1$ Å, se traduce como una barrera menos limitante para eventos de cruce a lo largo de las direcciones paralelas a los ejes X y Y de la celda. Por tanto la difusión del metano en el plano XY estará determinada por la superficie envolvente de las celdas clivadas, a diferencia de lo que ocurriría en la zona interior, donde existe isotropía difusional.

Un estudio computacional publicado recientemente por Zimmerman et al. ${ }^{179}$ para gases adsorbidos en una zeolita de tipo AFI, y que analiza perfiles de energía libre para metano y etano a diferentes concentraciones, permite observar una dependencia con la concentración de tal magnitud que se llega a generar una capa 
de líquido sobre la superficie. En comparación con nuestros resultados, el efecto de la superficie en la dependencia de la adsorción con la concentración se hace evidente.

La imagen microscópica que se puede obtener a partir de los datos obtenidos sugiere un proceso de adsorción inicialmente limitado por el área externa disponible, donde una fracción de partículas en la fase gaseosa interacciona con regiones superficiales favorables energéticamente, ubicadas entre los átomos de oxígeno parcialmente expuestos para despues deslizarse hacia el interior del cristal, y una fracción mayor de moléculas que logran permear la superficie a través de las zonas libres no atractivas.

La adsorción inicial es seguida por la transferencia de masa a lo largo de la coordenada que define el gradiente de concentración, inicialmente influenciada por la topología de las celdas parcialmente clivadas, hasta alcanzar el interior del cristal donde la movilidad del adsorbato estará influenciado por las zonas de interacción superficial favorable y las limitantes entrópicas que presentan las ventanas de 8 unidades T.

\subsubsection{Permeabilidad Superficial}

Los perfiles mostrados en la figura 6.3 muestran el proceso transitorio de adsorción de metano en la lámina de zeolita modelada, proveyendo la información acerca del flujo de moléculas que ingresan al interior de la estructura cristalina. Los perfiles se ajustan a funciones exponenciales sencillas, con forma general $\triangle N(t)=N_{0}-N_{0} \exp (-t / \tau)$, y $(\tau)$ constantes de tiempo facilmente obtenibles a partir del ajuste. Las condiciones de equilibrio representadas por las lineas horizontales en la figura 6.3 corresponden a la situación de flujo igual a cero a través de la superficie. Esta zona de equilibrio se utilizó para generar los datos de la isoterma correspondientes al equilibrio existente entre la fase gaseosa y la fase adsorbida, representada gráficamente en la figura 6.4.

Utilizando la definición planteada por la ecuación 6.3 y los datos acumulados en las trayectorias dinámicas podemos calcular los valores para la permeabilidad superficial, los cuales varían entre 2,5 a $12 \frac{\mathrm{m}}{\mathrm{s}}$, incrementándose proporcionalmente con el aumento de la concentración de la fase gaseosa. Los valores de $\alpha_{s}$ calculados se representan graficamente en la figura 6.7.

En un trabajo recientemente publicado por Thompho et al. ${ }^{182}$ se presentan los resultados de un estudio similar de dinámica molecular en condiciones de noequilibrio en el sistema silicalita-1/metano, utilizando un modelo explícito para los grupos silanol superficiales. Coincide con el presente trabajo el tamaño de la celda de simulación utilizada, con obvias diferencias dadas por las características topológicas de los materiales: sistema de canales unidimensionales con aperturas 


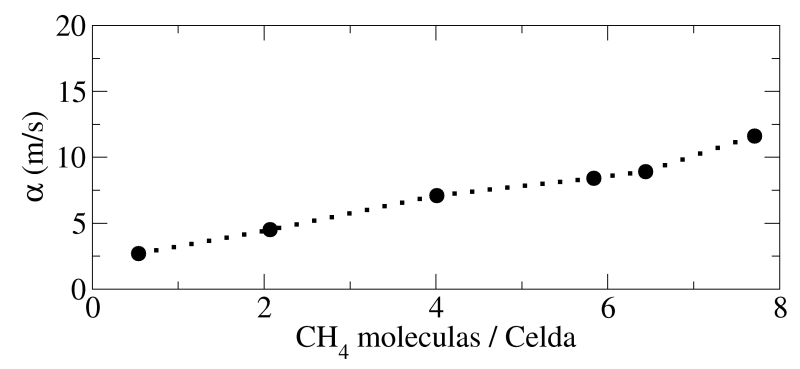

Figura 6.7: Valores para la permeabilidad superficial $\alpha_{s}$ calculados a partir de los datos obtenidos de las simulaciones de DM y utilizando la definición dada por la ecuación 6.3. La linea punteada se traza para evidenciar la tendencia incremental de $\alpha_{s}$ respecto a la concentración.

de 10 unidades $\mathrm{T}$ en el caso de la silicalita-1 y sistema tridimensional de celdas unidas por ventanas de 8 unidades $\mathrm{T}$ en nuesto caso.

Sus resultados consisten en resistencias superficiales a la transferencia de masa dependientes del tiempo, $\alpha_{s}(t)$, con valores en el rango [5-28] $\frac{m}{s}$. Estos valores están dentro del mismo orden de magnitud a los encontrados en el presente trabajo, pudiendo adjudicarse las diferencias a las condiciones menos restrictivas entrópicamente en el sistema poroso de la silicalita-1.

Una segunda fuente de información, valiosa para la validación de nuestros resultados, viene del trabajo de Schüring et al. ${ }^{194}$ En su publicación, se describe el estudio del proceso de transferencia de masa superficial desde un punto de vista analítico, desarrollando para ello un modelo simplificado capaz de cuantificar la resistencia superficial en condiciones de equilibrio $\left(\alpha_{s}^{E}\right)$. El modelo correspondería al experimento "tracer-exchange", caracterizado por el flujo neto igual a cero a través de la superficie, de acuerdo a la relación

$$
\alpha_{s}^{E}=P_{\text {Enter }} \frac{\nu}{K}
$$

donde $P_{\text {Enter }}$ se define como la relación entre el número de partículas que efectivamente alcanzan el interior del sistema cristalino y el número total de moléculas que inciden en la superficie en un cierto período de tiempo, ${ }^{195} \nu=158$ $\frac{m}{s}$ es el valor promedio de la velocidad terminal de una molécula de metano de masa $\mathrm{m}$ viajando en un sistema estabilizado a la temperatura T, y $K$ representa la relación entre la densidad molecular en la zona superficial limitante y la fase puramente gaseosa.

Los valores de $\alpha_{s}^{E}$ obtenidos en el trabajo de Schüring et al. para la adsorción en equilibrio de 3 moléculas de metano por celda unidad de zeolita Si-LTA es 
de $1,5 \frac{\mathrm{m}}{\mathrm{s}}$ a $300 \mathrm{~K}$. El valor del parámetro $P_{\text {enter }}$ a las condiciones especificadas anteriormente es de 0,05 . Penter calculado a partir de nuestras simulaciones a condiciones similares produce un valor de 0,17 , más de tres veces mayor que el de Schüring. El parámetro $K$ es, sin embargo, comparable en los dos casos, con valores de 5,4 y 4,9 , correspondientes a Schüring y a los resultados obtenidos en nuestro trabajo respectivamente.

Con los parámetros calculados a partir de las trayectorias de DM presentes, el valor de $\alpha_{s}$ obtenido sería de $5.47 \frac{\mathrm{m}}{\mathrm{s}}$. Las diferencias entre los valores pueden ser racionalizadas si tenemos en cuenta que nuestro modelo proporciona zonas superficiales preferenciales para la adsorción de metano, tal como se detalló en la sección anterior. Estas zonas no existirían en el modelo de Schüring et al. y como consecuencia sus valores de $\alpha_{s}$ indicarían un proceso de adsorción superficial más restringido.

Los valores obtenidos para el rango de concentraciones estudiado se presenta en la figura 6.8. La característica más sobresaliente sería así la tendencia de aumento en el valor de $\alpha_{s}^{E}$ respecto a la concentración.

Basados en los valores de $\alpha_{s}$ calculados, hemos determinado las condiciones para las cuales la barrera superficial tendría relevancia en relación con las resistencias difusionales intracristalinas. La resistencia total a la transferencia de masa, tal como la definen Kärger y Ruthven ${ }^{148}$ estará dada por la relación

$$
\tau=\tau_{\text {Diff }}+\tau_{\text {Barr }}=\frac{R^{2}}{15 D}+\frac{R}{3 \alpha_{s}}
$$

la cual se obtiene del primer momento estadístico de las curvas de adsorción transitoria, siendo $\mathrm{R}$ el radio de una esfera análoga a la forma de las partículas de adsorbente. El caso límite de control difusivo intracristalino y superficial está dado por $\tau_{\text {Diff }}=\frac{R^{2}}{15 D}$ y $\tau_{\text {Barr }}=\frac{R}{3 \alpha_{s}}$ respectivamente. Las resistencias superficiales tendrían relevancia en el caso de que $\frac{\tau_{\text {Diff }}}{\tau_{\text {Barr }}}=\frac{5 D}{R \alpha_{s}}>1$ o para el caso de tamaños de partícula muy pequeños. Utilizando valores de coeficientes de difusión calculados anteriormente para el mismo sistema Si-LTA/metano, ${ }^{196}$ cercanos a $6 \times 10^{-9} \frac{m^{2}}{s}$, y con los valores de $\alpha_{s}$ obtenidos aquí, se encuentra que los efectos de retención superficial vendrían a ser relevantes para partículas con tamaños $\mathrm{R}$ en el orden de $10 \AA$, los cuales estarían en las dimensiones de una celda unidad.

Este resultado está en perfecta consonancia con la evidencia de resistencias superficiales en materiales nanoporosos reportados recientemente. ${ }^{174,175,183}$ Estos trabajos muestran que las resistencias reales en sistemas cristalinos nanoporosos deben ser adjudicadas a imperfecciones estructurales severas del material, siendo necesario un bloqueo casi total de los accesos superficiales para la medición de barreras superficiales relevantes para la cinética global del proceso de adsorción. 


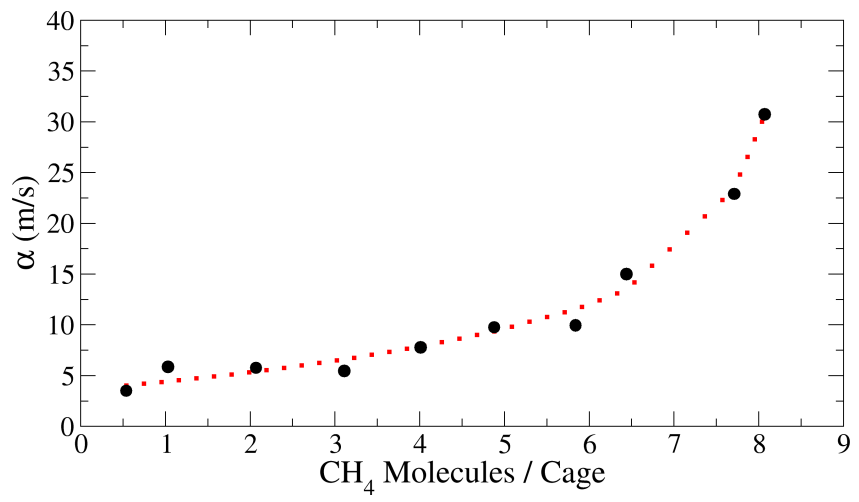

Figura 6.8: Valores de permeabilidad superficial $\alpha_{s}^{E}$ calculados a partir de las trayectorias de DM en conjunto con la expresión analítica (Ecuación 6.4) de Schüring et al. ${ }^{194}$ La linea punteada permite seguir la tendencia de los valores de $\alpha_{s}^{E}$.

\subsection{Conclusiones}

La técnica de la DM ha sido utilizada para obtener detalles a nivel microscópico del proceso de adsorción de metano en un modelo de zeolita Si-LTA con superficie compuesta por grupos silanol terminales explícitos. Partiendo de las trayectorias de la simulación se han obtenido histogramas con las densidades poblacionales en las diferentes regiones del sistema, así como mapas tridimensionales de ocupación. A partir de estos datos estadísticos de ocupación se han generado perfiles bidimensionales para la energía libre del adsorbato en las diferentes regiones del sistema.

Los mapas de ocupación 2D han permitido localizar zonas preferenciales de interacción en la superficie, ubicadas entre los oxígenos parcialmente expuestos pertenecientes a los grupos hidroxilo superficiales. En estas regiones se lleva a cabo la primera etapa de adsorción, la cual es seguida por la transferencia de masa a lo largo de la coordenada $Z$, inicialmente influenciada por las celda- $\alpha$ parcialmente clivadas y después guiado por las características topológicas internas propias del material.

La permeabilidad superficial, calculado ya sea usando los resultados de las simulaciones obtenidos aquí o la expresión analítica de Schüring et al. muestra un incremento sustancial con la concentración, comportamiento visto experimentalmente.

Con base en los valores obtenidos para $\alpha_{s}$ y tomando valores para el coeficiente de difusión en el mismo sistema, Si-LTA/metano, calculados anteriormente, se ha mostrado que las barreras superficiales serían relevantes para el proce- 
so global de transferencia de masa en partículas con diámetros del orden de la celda unidad. Este resultado está en consonancia con la evidencia experimental sobre la resistencia superficial en nanoporos, ${ }^{197}$ y con análisis computacionales y analíticos obtenidos subsecuentemente, ${ }^{198}$ donde se muestra que las resistencias superficiales en cristales nanoporosos "reales" deben estar dadas por desviaciones estructurales severas, probablemente por el bloqueo casi total de las ventanas superficiales.

El modelamiento atomístico de la superficie entre la fase cristalina y la atmósfera gaseosa que la rodea no conlleva a barreras superficiales experimentalmente observadas en cristales "reales", los cuales tienen dimensiones de cientos de miles de celdas unidad. Este resultado puede ser racionalizado de la siguiente forma: si asumimos que la resistencia ofrecida por las aperturas superficiales tiene alguna significancia en el proceso de captura o descarga, su influencia debe estar en competencia con la dinámica interna, descrita por saltos que la molécula ejecuta en su desplazamineto intracristalino. La probabilidad de estos saltos para atravesar las ventanas externas (asumiendo que todas o un fracción muy alta están abiertas) tendría que ser, por lo tanto, mucho menor a la probabilidad de saltos ejecutados en el interior, por ejemplo 1/100, lo cual correspondería a energías de activación elevadas, las cuales sin embargo no son observadas.

Las resistencias superficiales estimadas en este estudio pueden ser de relevancia inmediata para sistemas adsorbato/adsorbente que consistan de capas muy delgadas de material nanoporoso. ${ }^{199}$ En segundo lugar, los estimativos obtenidos son de relevancia cuando se considera la transferencia de masa en zeolitas mesoporosas. ${ }^{200-202}$ En estos materiales, la transferencia de masa entre el espacio puramente intracristalino y la atmósfera circundante es acelerada por la transferencia entre microporos y mesoporos. Bajo estas condiciones el valor finito de la velocidad de transferencia de masa en la interfase interna entre mesoporos y microporos tendría relevancia, lo cual resalta la relevancia de los estimativos que se hacen en este trabajo. 
Rol de la Superficie en el Proceso de Adsorción de Metano en la Zeolita 


\section{Capítulo 7}

\section{Conclusiones}

La difusión de adsorbatos en materiales nanoporosos representa un desafío desde varias perspectivas. Primero, se diferencia de manera fundamental de la difusión en medios homogéneos en términos cualitativos y cuantitativos, por cuanto los mecanismos físicos que se suceden en el proceso involucran interacciones con superficies, lo cual lo convierte en un fenómeno cualitativamente diferente, con consecuencias visibles cuantitativamente, en algunos casos de órdenes de magnitud si se compara con la difusión en fases homogéneas.

Como consecuencia, estas mismas peculiaridades lo convierten en un fenómeno especialmente interesante, desde la perspectiva de la necesidad de comprender los mecanismos físicos que la gobiernan, pero además útil en extremo, ya que tiene el potencial para ser aprovechado en múltiples procesos, ya sean catalíticos, de separación, electrónica, medicina, etc.

En los capítulos iniciales de esta tesis se ha querido plasmar la evolución del entendimiento y del estudio de los fenómenos de difusión, inicialmente desde su perspectiva puramente histórica (Capítulo I), para luego presentar el desarrollo de las teorías propuestas a principios del siglo pasado, y que representan las bases para la posterior aparición de las técnicas computacionales (Capítulos II y III) que son utilizadas en el presente trabajo.

- El objeto del Capítulo IV es el de presentar la información existente sobre los campos de fuerza más utilizados en estudios atomísticos aplicados a materiales zeolíticos. Con este fin en mente, se analizaron a fondo una serie de potenciales desarrollados para la modelización de este tipo de estructuras, modelos que discrepan en cuanto a complejidad funcional y en el proceso de parametrización. Estos campos de fuerza se evalúan en cuanto a su transferabilidad, comparando mediciones estructurales, mecánicas y dieléctricas experimentales, con resultados calculados computacionalmente. 
Como conclusión de este apartado, podemos decir que la gran variedad de campos de fuerza existentes para el modelamiento de las interacciones en zeolitas hace que la elección de uno de ellos para un propósito determinado sea como mínimo difícil. Dentro de los potenciales estudiados, tenemos modelos como el Core-Shell, que son indicados para la reproducción o predicción de estructuras utilizando técnicas como Lattice-Dynamics, que sin embargo no representan una opción computacionalmente eficiente para simulaciones de tipo Dinámica-Molecular.

Así, los potenciales modelados como iones rígidos, con cargas puntuales no formales, son la mejor alternativa para estudiar la dinámica de especies adsorbidas en zeolitas. Dentro de este subgrupo, hemos seleccionado el potencial BKS para nuestras simulaciones debido a dos características principales: su extrema simplicidad funcional y su alto nivel de coincidencia en la predicción de términos estructurales, como parámetros de red.

La simplicidad funcional, dada por los términos de Buckhingham que describen las interacciones $\mathrm{Si}-\mathrm{O}$ y $\mathrm{O}-\mathrm{O}$, permite llevar a cabo simulaciones del orden de cientos de nanosegundos, lo que permite ser usado para el estudio de la difusión de los adsorbatos más voluminosos, y por tanto con coeficientes de difusión muy bajos, que llegan a tocar los límites de uso de la técnica de la Dinámica Molecular.

- En el Capítulo IV se estudia la influencia del potencial interatómico respecto a la dinámica de la red. Este efecto se analizó empleando dos poteciales diferentes, ampliamente utilizados, con el objetivo de resaltar el efecto del potencial sobre los parámetros estructurales del material y sobre la movilidad de las moleculas adsorbidas. El análisis de los datos se basa en la identificación de factores estructurales asociados al tipo de potencial utilizado para la representación de las interacciones internas de la red.

Como conclusión de este estudio se da la identificación de los diámetros críticos de las ventanas y sus fluctuaciones con respecto a la temperatura, fluctuaciones que por supuesto afectan la movilidad de los adsorbatos de manera apreciable. La variación del diámetro crítico de las ventanas no se ve afectada por la temperatura en alto grado. Estas fluctuaciones, están en el orden de $¡ 1$ Å, que sin embargo sumada a la exitación propia de los grados de libertad translacionales y rotacionales de los adsorbatos, resulta en un aumento de la movilidad intracristalina, en comparación con una red fija.

- En el Capítulo V, se usa la técnica de la dinámica molecular para el estudio de la movilidad de hidrocarburos de bajo peso molecular, C1-C3, adsorbidos en tres estructuras zeolíticas puramente silíceas ITQ-29(Si-LTA), 
ITQ-32(Si-IHW) y ITQ-3(Si-ITE). Punto común a estas estructuras es la existencia de ventanas de 8 unidades tetraédricas que comunica los poros y canales, y que representan las barreras limitante para el proceso de difusión.

Los coeficientes de difusión en estas estructuras muestran una correlación inversa entre el diámetro cinético de los adsorbatos y el diámetro crítico de las ventanas, confirmando así medidas experimentales que identifican la apertura de las ventanas como la barrera limitante, barrera energéticamente detallada a partir de los perfiles de ocupación y de las energías libres resultantes.

- En el capítulo VI, se detallado a nivel microscópico el proceso de adsorción de metano en un modelo de zeolita si-LTA, el cual se ha modelado incluyendo los grupos silanol terminales, a partir de cálculos de Dinámica molecular. Partiendo de las trayectorias de la simulación se han obtenido histogramas con las densidades poblacionales en las diferentes regiones del sistema, así como mapas tridimensionales de ocupación. El mecanismo de adsorción superficial se ha obtenido a partir de los perfiles de ocupación y de las energías libres calculadas a partir de ellos.

Como conclusión, y partiendo de los valores de permeabilidad superficial $\alpha_{s} \mathrm{y}$ coeficiente de difusividad, se sugiere que las barreras superficiales serían relevantes para el proceso global de transferencia de masa en partículas con diámetros muy pequeños, del orden de unas pocas celdas unidad. Nuestros resultados están en perfecta consonancia con evidencia experimental sobre la resistencia superficial en nanoporos, y con análisis computacionales y analíticos obtenidos subsecuentemente, donde se muestra que las resistencias superficiales en cristales nanoporosos "reales" deben estar dadas por desviaciones estructurales muy severas, debidas probablemente a un bloqueo casi total de los puntos de acceso superficiales. Las trayectorias dinámicas obtenidas para el proceso de adsorción superficial entre la fase cristalina y la atmósfera gaseosa que la rodea, no conllevan a barreras superficiales experimentalmente observadas en cristales "reales", los cuales tienen dimensiones de cientos de miles de celdas unidad. 


\section{Apéndice A}

\section{Parámetros Estructurales, Mecánicos y Dieléctricos de Estructuras Seleccionadas}

A continuación se presentan datos obtenidos a través de la técnica de la Dinámica de Red (Lattice Dynamics) para una selección de estructuras, en comparación con datos experimentales. 


\section{A.1. Cuarzo- $\alpha$ : Parámetros Calculados y Experimenta- les}

Tabla A.1: Cuarzo- $\alpha$ : Parámetros Estructurales

\begin{tabular}{|c|c|c|c|c|c|c|c|}
\hline Potential & $\mathbf{a}(\AA)$ & 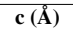 & $\alpha=\beta=\gamma($ Grad $)$ & $S i-O(\AA)$ & Si-Si $(\AA)$ & $\angle S i-O-S i$ (Grad) & $\angle O-S i-O($ Grad $)$ \\
\hline SLC & 4.867 & 5.375 & 90.00 & $\begin{array}{c}1.605-1.611 \\
1.608\end{array}$ & 3.035 & $\begin{array}{c}141.357-141.359 \\
141.4\end{array}$ & $\begin{array}{c}108.385-110.963 \\
109.475\end{array}$ \\
\hline SS96 & 4.988 & 5.506 & 90.00 & $\begin{array}{c}1.614-1.617 \\
1.616\end{array}$ & 3.105 & $\begin{array}{c}147.812-147.814 \\
147.813\end{array}$ & $\begin{array}{c}108.415-111.076 \\
109.475\end{array}$ \\
\hline SS97 & 4.890 & 5.452 & 90.00 & $\begin{array}{c}1.637-1.641 \\
1.639\end{array}$ & 3.065 & $\begin{array}{c}138.458-138.461 \\
138.460\end{array}$ & $\begin{array}{c}107.338-111.273 \\
\text { SLC }\end{array}$ \\
\hline Gale & 4.899 & 5.402 & 90.00 & $\begin{array}{c}1.605-1.615 \\
1.610\end{array}$ & 3.054 & $\begin{array}{c}142.963-142.966 \\
142.964\end{array}$ & $\begin{array}{c}108.342-110.586 \\
109.472\end{array}$ \\
\hline SC1 & 4.902 & 5.408 & 90.00 & $\begin{array}{c}1.627-1.625 \\
1.6256\end{array}$ & 3.057 & $\begin{array}{c}140.034-140.036 \\
140.035\end{array}$ & $\begin{array}{c}108.373-110.958 \\
109.474\end{array}$ \\
\hline $\mathrm{SC} 2$ & 4.948 & 5.360 & 90.00 & $\begin{array}{l}1.605 \\
1.605\end{array}$ & 3.061 & $\begin{array}{c}145.000-145.003 \\
145.001\end{array}$ & $\begin{array}{c}109.469-109.473 \\
109.471\end{array}$ \\
\hline JC1 & 4.814 & 5.378 & 90.00 & $\begin{array}{c}1.606-1.616 \\
1.611\end{array}$ & 3.020 & $\begin{array}{c}139.120-139.122 \\
139.121\end{array}$ & $\begin{array}{c}107.192-110.953 \\
109.479\end{array}$ \\
\hline JC2 & 5.105 & 5.631 & 90.00 & $\begin{array}{l}1.603 \\
1.603\end{array}$ & 3.168 & 162.348 & $\begin{array}{c}104.430-115.867 \\
109.543\end{array}$ \\
\hline DSQFG & 4.919 & 5.311 & 90.00 & $\begin{array}{l}1.604 \\
1.604\end{array}$ & 3.043 & $\begin{array}{c}142.995-142.997 \\
142.996\end{array}$ & $\begin{array}{c}109.470-109.473 \\
109.471\end{array}$ \\
\hline JA & 4.442 & 5.256 & 90.00 & $\begin{array}{c}1.594-1.612 \\
1.603\end{array}$ & 2.890 & $\begin{array}{c}128.783-128.785 \\
128.783\end{array}$ & $\begin{array}{c}102.057-116.778 \\
109.495\end{array}$ \\
\hline АHCM & 4.481 & 5.136 & 90.00 & $\begin{array}{c}1.536-1.552 \\
1.544\end{array}$ & 2.863 & $\begin{array}{c}136.025-136.028 \\
136.026\end{array}$ & $\begin{array}{c}104.284-115.753 \\
109.425\end{array}$ \\
\hline BKS & 4.941 & 5.449 & 90.00 & $\begin{array}{c}1.597-1.605 \\
1.601\end{array}$ & 3.081 & $\begin{array}{c}148.408-148.410 \\
148.409\end{array}$ & $\begin{array}{c}107.809-114.827 \\
109.463\end{array}$ \\
\hline Vessal & 5.0975 & 5.6794 & 90.00 & $\begin{array}{l}1.607 \\
1.607\end{array}$ & 3.175 & 162.219 & $\begin{array}{c}104.878-115.934 \\
109.541\end{array}$ \\
\hline PMM06 & 4.9387 & 5.4432 & 90.00 & $\begin{array}{c}1.594-1.611 \\
1.603\end{array}$ & 3.081 & $\begin{array}{c}147.981-147.983 \\
147.982\end{array}$ & $\begin{array}{c}107.702-115.036 \\
109.451\end{array}$ \\
\hline PMM08 & 4.911 & 5.4045 & 90.00 & $\begin{array}{c}1.631-1.634 \\
1.633\end{array}$ & 3.063 & $\begin{array}{c}139.450-139.452 \\
139.451\end{array}$ & $\begin{array}{c}108.132-110.249 \\
109.474\end{array}$ \\
\hline TTAM & 4.941 & 5.4489 & 90.00 & $\begin{array}{c}1.597-1.605 \\
1.601\end{array}$ & 3.081 & $\begin{array}{c}148.408-148.410 \\
148.409\end{array}$ & $\begin{array}{c}107.809-114.826 \\
109.463\end{array}$ \\
\hline $\operatorname{Exp}^{203}$ & 4.916 & 5.4054 & 90.00 & 1.6092 & 3.058 & 143.7 & 109.24 \\
\hline $\operatorname{Exp}^{125}$ & 4.9134 & 5.4052 & 90.00 & $1.608-1.610$ & & 143.6 & $108.7-110.5$ \\
\hline
\end{tabular}


Tabla A.2: Cuarzo- $\alpha$ : PropiedadesMecánicas

\begin{tabular}{lccccccc}
\hline Potential & Bulk Modulus (GPa) & \multicolumn{6}{c}{ Elastic Constants (GPa) } \\
& $K$ & $\mathbf{C}_{11}$ & $\mathbf{C}_{33}$ & $\mathbf{C}_{44}$ & $\mathbf{C}_{66}$ & $\mathbf{C}_{14}$ & $\mathbf{C}_{13}$ \\
\hline SLC & 44.41 & 94.66 & 112.57 & 49.68 & 39.94 & -15.79 & 17.89 \\
SS96 & 42.01 & 84.56 & 96.34 & 41.07 & 36.53 & -13.70 & 23.94 \\
SS97 & 45.35 & 75.89 & 105.64 & 38.14 & 26.10 & -7.85 & 28.99 \\
Gale & 44.96 & 97.00 & 105.23 & 47.99 & 40.52 & -16.28 & 18.60 \\
SC1 & 43.37 & 91.80 & 113.21 & 48.57 & 38.26 & -17.20 & 16.71 \\
SC2 & 38.25 & 103.35 & 84.85 & 54.66 & 39.80 & -16.97 & 3.90 \\
JC1 & 121.11 & 202.32 & 212.93 & 63.07 & 67.99 & 0.0001 & 86.27 \\
JC2 & 43.72 & 70.52 & 92.41 & 31.91 & 22.94 & -6.27 & 30.01 \\
DS & 0.000 & 31.71 & 28.13 & 32.92 & 13.62 & -21.17 & -22.56 \\
JA & 37.99 & 62.79 & 156.10 & 17.43 & 20.43 & 15.56 & 15.27 \\
AHCM & 43.54 & 69.27 & 176.32 & 36.30 & 23.04 & 1.658 & 24.65 \\
BKS & 40.10 & 90.55 & 107.07 & 50.27 & 41.22 & -17.66 & 15.24 \\
Vessal & 199.16 & 318.10 & 301.87 & 78.61 & 99.49 & -0.0001 & 154.46 \\
PMM06 & 37.46 & 88.41 & 103.54 & 49.31 & 40.38 & -18.33 & 11.03 \\
PMM08 & 34.62 & 72.88 & 97.59 & 46.86 & 29.97 & -13.34 & 11.77 \\
TTAM & 33.49 & 71.34 & 93.98 & 40.53 & 30.73 & -13.76 & 12.73 \\
\hline Exp & 38.98 & 86.83 & 105.98 & 58.26 & 39.87 & -18.06 & 11.93 \\
\hline
\end{tabular}

Tabla A.3: Cuarzo- $\alpha$ : Propiedades Dieléctricas

\begin{tabular}{|c|c|c|}
\hline Potential & $\begin{array}{l}\text { Static Dielectric } \\
\text { Constant } \epsilon_{11}^{0}-\epsilon_{33}^{0}\end{array}$ & $\begin{array}{c}\text { High Frequency } \\
\text { Dielectric Constant } \epsilon_{11}^{\infty}-\epsilon_{33}^{\infty}\end{array}$ \\
\hline SLC & $4.58-4.88$ & $2.07-2.09$ \\
\hline SS96 & $4.07-4.42$ & $1.75-1.76$ \\
\hline SS97 & $4.47-4.65$ & $1.84-1.86$ \\
\hline Gale & $4.81-5.29$ & $1.98-1.99$ \\
\hline SC1 & $3.32-3.53$ & $1.91-1.93$ \\
\hline SC2 & $4.14-4.26$ & $3.09-3.21$ \\
\hline JC1 & $5.83-6.12$ & $2.14-2.17$ \\
\hline $\mathrm{JC2}$ & $3.90-4.07$ & \\
\hline DSQFG & $1.00-1.00$ & \\
\hline $\mathbf{J A}$ & $2.33-2.34$ & -- \\
\hline АHCM & $2.31-2.36$ & \\
\hline BKS & $1.95-1.99$ & \\
\hline Vessal & $3.68-3.83$ & - \\
\hline PMМ06 & $2.25-2.32$ & $\longrightarrow$ \\
\hline PMM08 & $2.78-2.84$ & $1.423-1.425$ \\
\hline TTAM & $2.11-2.17$ & $\longrightarrow$ \\
\hline $\operatorname{Exp}^{204}$ & $4.51-4.60$ & $2.4-2.4$ \\
\hline
\end{tabular}




\section{A.2. Cuarzo- $\beta$ : Parámetros Calculados y Experimenta- les}

Tabla A.4: Cuarzo- $\beta$ : Parametros de Red

\begin{tabular}{|c|c|c|c|c|c|c|}
\hline Potential & $\mathbf{a}[\AA]$ & c $[\AA]$ & $S i-O[\AA]$ & $S i-S i[\AA]$ & $\angle S i-O-S i$ & $\angle O-S i-O$ \\
\hline SLC & 5.00 & 5.50 & 1.595 & 3.102 & 152.840 & $\begin{array}{c}111.699-106.941 \\
109.483\end{array}$ \\
\hline SS96 & 5.08 & 5.58 & 1.609 & 3.148 & 156.054 & $\begin{array}{c}109.133-109.904 \\
109.472\end{array}$ \\
\hline SS97 & 5.11 & 5.60 & 1.625 & 3.164 & 153.6 & $\begin{array}{c}107.621-110.980 \\
109.477\end{array}$ \\
\hline Gale & 5.02 & 5.52 & 1.599 & 3.112 & 153.6 & 109.5 \\
\hline SC1 & 5.05 & 5.55 & 1.612 & 3.132 & 152.702 & $\begin{array}{c}106.776-111.746 \\
109.484\end{array}$ \\
\hline $\mathrm{SC2}$ & 5.10 & 5.56 & 1.609 & 3.154 & 156.987 & $\begin{array}{c}108.087-110.591 \\
109.475\end{array}$ \\
\hline JC1 & 5.10 & 5.63 & 1.603 & 3.168 & 162.348 & $\begin{array}{c}104.430-115.866 \\
109.543\end{array}$ \\
\hline JC2 & 5.01 & 5.51 & 1.595 & 3.105 & 153.469 & $\begin{array}{c}107.524-111.202 \\
109.478\end{array}$ \\
\hline DSQFG & 5.06 & 5.56 & 1.604 & 3.136 & 155.597 & $\begin{array}{c}109.469-109.474 \\
109.471\end{array}$ \\
\hline JA & 5.04 & 5.59 & 1.598 & 3.136 & 157.545 & $\begin{array}{c}108.290-111.473 \\
109.478\end{array}$ \\
\hline AHCM & 4.88 & 5.40 & 1.542 & 3.030 & 158.438 & $\begin{array}{c}107.551-112.278 \\
109.484\end{array}$ \\
\hline BKS & 5.07 & 5.59 & 1.600 & 3.147 & 158.833 & $\begin{array}{c}107.129-112.581 \\
109.488\end{array}$ \\
\hline Vessal & 5.12 & 5.62 & 1.605 & 3.172 & 162.093 & $\begin{array}{c}104.421-115.518 \\
109.537\end{array}$ \\
\hline PMM06 & 5.07 & 5.60 & 1.602 & 3.149 & 158.851 & $\begin{array}{c}107.145-112.616 \\
109.488\end{array}$ \\
\hline PMM08 & 5.12 & 5.62 & 1.625 & 3.171 & 154.573 & $\begin{array}{c}108.507-110.266 \\
109.473\end{array}$ \\
\hline TTAM & 4.94 & 5.45 & 1.601 & 3.081 & 148.409 & $\begin{array}{c}107.809-114.827 \\
109.463\end{array}$ \\
\hline $\operatorname{Exp}^{125126}$ & 5.01 & 5.47 & $\begin{array}{c}- \\
1.616 \\
\end{array}$ & $\begin{array}{c}- \\
3.098 \\
\end{array}$ & $\begin{array}{c}- \\
146.930 \\
\end{array}$ & $\begin{array}{c}101.307-116.134 \\
109.585 \\
\end{array}$ \\
\hline
\end{tabular}


Tabla A.5: Cuarzo- $\beta$ : Propiedades Mecánicas

\begin{tabular}{lcccccc}
\hline Potential & ${\text { Bulk Modulus }(\mathbf{G P a})^{*}}$ & \multicolumn{5}{c}{ Elastic Constants $^{(\mathbf{G P a}}$} \\
& & $\mathbf{C}_{11}$ & $\mathbf{C}_{33}$ & $\mathbf{C}_{44}$ & $\mathbf{C}_{66}$ & $\mathbf{C}_{13}$ \\
\hline SLC & 155.38 & 221.67 & 229.92 & -6.71 & 59.43 & 132.78 \\
SS96 & 175.17 & 229.16 & 232.92 & 13.91 & 49.70 & 159.42 \\
SS97 & 159.72 & 209.27 & 215.60 & -186.50 & 45.79 & 145.21 \\
Gale & 194.74 & 260.89 & 269.91 & 1.49 & 60.69 & 174.47 \\
SC1 & 186.00 & 255.48 & 264.16 & -13.51 & 62.12 & 164.85 \\
SC2 & 75.97 & 99.12 & 105.01 & -1.65 & 20.82 & 67.76 \\
JC1 & 121.11 & 202.32 & 212.93 & 63.07 & 67.99 & 86.27 \\
JC2 & 143.01 & 185.75 & 194.06 & -94.25 & 40.11 & 131.42 \\
DSQFG & 123.86 & 169.63 & 175.56 & -0.0002 & 40.94 & 108.07 \\
AHCM & 178.38 & 232.85 & 239.96 & -107.85 & 51.57 & 165.03 \\
JA & 135.55 & 172.14 & 183.11 & 42.96 & 35.45 & 128.20 \\
BKS & 172.45 & 237.95 & 234.20 & 11.60 & 59.30 & 152.84 \\
Vessal & 200.91 & 319.64 & 295.76 & 77.47 & 99.68 & 158.40 \\
PMM06 & 136.90 & 202.92 & 196.95 & 8.32 & 58.38 & 115.48 \\
PMM08 & 144.71984 & 199.5813 & 199.6317 & -64.0906 & 49.4099 & 127.4180 \\
TTAM & 138.34154 & 194.3518 & 192.2987 & 12.0379 & 50.5642 & 121.1993 \\
\hline Exp $^{205}$ & $42.97^{206}$ & 118.4 & 107.0 & 35.8 & 49.7 & 32.0 \\
\hline
\end{tabular}

Tabla A.6: Cuarzo- $\beta$ : Propiedades Dieléctricas

\begin{tabular}{|c|c|c|}
\hline Potential & $\begin{array}{l}\text { Static Dielectric } \\
\text { Constant } \epsilon_{11}^{0}-\epsilon_{33}^{0}\end{array}$ & $\begin{array}{c}\text { High Frequency } \\
\text { Dielectric Constant } \epsilon_{11}^{\infty}-\epsilon_{33}^{\infty}\end{array}$ \\
\hline SL & $4.66-5.06$ & $1.99-2.01$ \\
\hline SS96 & $4.13-4.59$ & $1.71-1.72$ \\
\hline SS97 & $5.23-5.23$ & $1.75-1.76$ \\
\hline Gale & $5.04-5.70$ & $1.91-1.92$ \\
\hline SC1 & $3.33-3.58$ & $1.83-1.84$ \\
\hline $\mathrm{SC2}$ & $10.09-10.60$ & $6.99-7.64$ \\
\hline JC1 & $6.84-7.17$ & $2.04-2.05$ \\
\hline JC2 & $3.90-4.07$ & - \\
\hline DSQFG & $1.00-1.00$ & \\
\hline АНСМ & $1.97-1.99$ & - \\
\hline JA & $1.77-1.86$ & 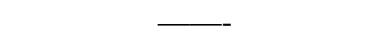 \\
\hline BKS & $1.87-1.89$ & - \\
\hline Vessal & $3.65-3.81$ & - \\
\hline РMМ06 & $2.12-2.14$ & - \\
\hline PMM08 & $2.69-2.85$ & $1.37-1.37$ \\
\hline TTAM & $1.97-1.98$ & 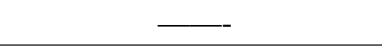 \\
\hline $\operatorname{Exp}^{207}$ & & \\
\hline
\end{tabular}




\section{A.3. Coesita: Parámetros Calculados y Experimentales}

Tabla A.7: Coesita: Parametros de Red

\begin{tabular}{|c|c|c|c|c|c|c|c|c|}
\hline Potential & $\mathbf{a}[\AA \mathbf{A}]$ & 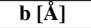 & 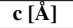 & $\beta[$ Deg] & $S i-O[\AA \mathbf{A}]$ & $S i-S i[\AA]$ & $\angle S i-O-S i$ & $\angle O-S i-O$ \\
\hline SLC & 7.0408 & 12.3075 & 7.1311 & 122.325 & $\begin{array}{c}1.569-1.626 \\
1.609\end{array}$ & $\begin{array}{c}2.991-3.138 \\
3.042\end{array}$ & $\begin{array}{c}180.000-133.870 \\
144.828\end{array}$ & $\begin{array}{c}105.313-112.341 \\
109.478\end{array}$ \\
\hline SS96 & 7.1875 & 12.5378 & 7.2557 & 122.803 & $\begin{array}{c}1.594-1.630 \\
1.620\end{array}$ & $\begin{array}{c}3.045-3.127 \\
3.092\end{array}$ & $\begin{array}{c}138.038-151.078 \\
147.907\end{array}$ & $\begin{array}{c}106.581-112.905 \\
109.459\end{array}$ \\
\hline SS97 & 7.2758 & 12.6772 & 7.3475 & 122.436 & $\begin{array}{c}1.607-1.641 \\
1.630\end{array}$ & $\begin{array}{c}3.092-3.215 \\
3.133\end{array}$ & $\begin{array}{c}180.000-141.033 \\
150.250\end{array}$ & $\begin{array}{c}106.122-112.398 \\
109.439\end{array}$ \\
\hline Gale & 7.0502 & 12.3936 & 7.1578 & 122.557 & $\begin{array}{r}1.569 \\
1.6\end{array}$ & $\begin{array}{r}3.011 \\
3 .\end{array}$ & $\begin{array}{l}135.082 \\
767\end{array}$ & $\begin{array}{l}112.647 \\
471\end{array}$ \\
\hline SC1 & 7.3208 & 12.7570 & 7.0590 & 130.555 & $\begin{array}{r}1.5893 \\
1.6\end{array}$ & $\begin{array}{r}3.001 \\
3 .\end{array}$ & $\begin{array}{l}180.000 \\
49\end{array}$ & $\begin{array}{l}61.188 \\
16\end{array}$ \\
\hline $\mathrm{SC2}$ & 7.23657 & 12.2867 & 7.1328 & 119.464 & $\begin{array}{r}1.608 \\
1.6\end{array}$ & 3.046 & $\begin{array}{l}143.127 \\
86\end{array}$ & $\begin{array}{r}110.075 \\
10\end{array}$ \\
\hline JC1 & 7.2558 & 12.4583 & 7.3218 & 119.405 & $\begin{array}{r}1.606 \\
1 .\end{array}$ & $\begin{array}{r}3.140 \\
3 .\end{array}$ & $\begin{array}{l}53.563 \\
63\end{array}$ & $\begin{array}{r}102.122 \\
109\end{array}$ \\
\hline $\mathrm{JC2}$ & 7.0548 & 12.4065 & 7.1359 & 124.9905 & $\begin{array}{r}1.605 \\
1.6\end{array}$ & $\begin{array}{r}2.991 \\
3.0\end{array}$ & $\begin{array}{l}133.813 \\
21\end{array}$ & $\begin{array}{l}114.000 \\
69\end{array}$ \\
\hline DSQF & 7.1262 & 12.2617 & 7.1225 & 119.747 & $\begin{array}{l}1.604 \\
1.604\end{array}$ & $\begin{array}{c}2.983-3.099 \\
3.064\end{array}$ & $\begin{array}{c}180.000-136.837 \\
147.994\end{array}$ & $\begin{array}{c}109.468-109.473 \\
109.471\end{array}$ \\
\hline JA & 6.7857 & 12.3966 & 7.2042 & 122.039 & $\begin{array}{c}1.586-1.609 \\
1.599\end{array}$ & $\begin{array}{c}2.980-3.138 \\
3.050\end{array}$ & $\begin{array}{c}135.192-155.396 \\
148.000\end{array}$ & $\begin{array}{c}104.553-117.211 \\
109.317\end{array}$ \\
\hline HC & 34 & 12.0307 & 7.00 & 121.697 & $\begin{array}{c}1.552-1.563 \\
1.544\end{array}$ & $\begin{array}{c}2.888-3.023 \\
2.968\end{array}$ & $\begin{array}{c}139.173-180.000 \\
150.757\end{array}$ & $\begin{array}{c}104.096-118.109 \\
109.305\end{array}$ \\
\hline BK & 7 & 12.4816 & 7.2 & 120.668 & $\begin{array}{c}1.579-1.611 \\
1.604\end{array}$ & $\begin{array}{c}3.077-3.124 \\
3.113\end{array}$ & $\begin{array}{c}180.000-144.463 \\
154.199\end{array}$ & $\begin{array}{c}105.339-115.686 \\
109.33\end{array}$ \\
\hline Vessal & 7.481 & 12.861 & 7.196 & 127.591 & $\begin{array}{c}1.530-1.757 \\
1.629\end{array}$ & $\begin{array}{c}3.117-3.221 \\
3.164\end{array}$ & $\begin{array}{c}137.823-180.000 \\
155.603\end{array}$ & $\begin{array}{c}96.643-129.516 \\
108.637\end{array}$ \\
\hline PMI & 7. & 12.4917 & 3 & 120.6046 & $\begin{array}{c}1.568-1.620 \\
1.606\end{array}$ & $\begin{array}{c}3.061-3.140 \\
3.118\end{array}$ & $\begin{array}{c}144.351-180.00 \\
154.233\end{array}$ & $\begin{array}{c}105.314-115.996 \\
109.307\end{array}$ \\
\hline 8 & 7. & 29 & 97 & 121.0625 & $\begin{array}{c}1.617-1.641 \\
1.632\end{array}$ & $\begin{array}{c}3.024-3.233 \\
3.104\end{array}$ & $\begin{array}{c}134.470-180.00 \\
146.721\end{array}$ & $\begin{array}{c}107.023-111.835 \\
109.465\end{array}$ \\
\hline TTAM & 7.213 & 12.752 & 7.430 & 120.767 & $\begin{array}{c}1.606-1.648 \\
1.638\end{array}$ & $\begin{array}{c}3.116-3.212 \\
3.175\end{array}$ & $\begin{array}{c}143.593-180.00 \\
153.740\end{array}$ & $\begin{array}{c}104.309-116.510 \\
109.314\end{array}$ \\
\hline $\operatorname{Exp}^{127}$ & 7.1357 & 12.3835 & 7.1859 & 120.375 & $1.6130-1.6158$ & 3.0894 & $137.29-149.54$ & $108.98-109.45$ \\
\hline
\end{tabular}


Tabla A.8: Coesita:Propiedades Mecánicas

\begin{tabular}{lccccc}
\hline Potential & Bulk Modulus (GPa) $^{*}$ & \multicolumn{4}{c}{ Elastic Constants [GPa] } \\
& & $\mathbf{C}_{11}$ & $\mathbf{C}_{33}$ & $\mathbf{C}_{44}$ & $\mathbf{C}_{66}$ \\
\hline SLC & 84.74 & 124.22 & 160.51 & 27.85 & 41.88 \\
SS96 & 76.75 & 133.55 & 149.69 & 27.09 & 34.09 \\
SS97 & 70.36 & 131.48 & 149.37 & 24.03 & 28.24 \\
Gale & 88.09 & 130.45 & 167.97 & 27.87 & 39.91 \\
SC1 & 168.66 & 262.47 & 282.02 & 24.62 & 13.22 \\
SC2 & 113.31 & 141.94 & 215.42 & 42.00 & 57.01 \\
JC1 & 71.94 & 118.34 & 127.68 & 20.47 & 24.79 \\
JC2 & 106.56 & 113.52 & 236.77 & 43.02 & 48.43 \\
DSQFG & - & -- & - & - & - \\
AHCM & 95.97 & 118.55 & 145.59 & -63.91 & -32.06 \\
JA & 120.70 & 154.30 & 204.93 & 4.26 & 2.57 \\
BKS & 124.33 & 178.10 & 252.21 & 36.98 & 32.04 \\
Vessal & 224.32 & 302.16 & 519.40 & 82.85 & 33.69 \\
PMM06 & 111.49 & 166.37 & 230.79 & 38.96 & 33.77 \\
PMM08 & 79.83 & 113.21 & 166.81 & 34.8789 & 47.3963 \\
TTAM & 99.67 & 142.49 & 199.41 & 26.37 & 21.49 \\
\hline Exp ${ }^{208-210}$ & $113.7-93.7$ & 91.41 & 245.09 & 55.77 & 68.35 \\
\hline
\end{tabular}

Tabla A.9: Coesita: Propiedades Dieléctricas

\begin{tabular}{lcc}
\hline Potential & $\begin{array}{c}\text { Static Dielectric } \\
\text { Constant } \epsilon_{x x}^{0}-\epsilon_{y y}^{0}-\epsilon_{z z}^{0}\end{array}$ & $\begin{array}{c}\text { High Frequency } \\
\text { Dielectric Constant } \epsilon_{11}^{\infty}-\epsilon_{33}^{\infty}\end{array}$ \\
\hline SLC & $2.232-2.234-2.245$ & $5.957-5.521-5.902$ \\
SS96 & $5.670-5.172-5.552$ & $1.885-1.884-1.893$ \\
SS97 & $5.255-4.922-5.171$ & $1.774-1.774-1.779$ \\
Gale & $7.356-6.213-7.040$ & $2.143-2.140-2.155$ \\
SC1 & $6.02-3.92-6.36$ & $2.18-2.12-2.25$ \\
SC2 & $5.22-5.61-5.20$ & $3.84-3.93-3.80$ \\
JC1 & $9.70-7.54-9.49$ & $2.32-2.32-2.35$ \\
JC2 & $4.021-3.997-3.936$ & - \\
DSQFG & $1.00-1.00-1.00$ & - \\
JA & $2.487-2.284-2.382$ & - \\
AHCM & $2.61-2.43-2.49$ & - \\
BKS & $2.228-2.182-2.184$ & - \\
Vessal & $9.011-5.304-5.835$ & - \\
PMM06 & $2.62-2.57-2.54$ & - \\
PMM08 & $3.33-3.18-3.25$ & - \\
TTAM & $2.44-2.37-2.36$ & - \\
\hline Exp 211 & 3.02 & - \\
\hline
\end{tabular}




\section{A.4. Si-LTA: Parámetros Calculados y Experimentales}

Tabla A.10: Si-LTA: Parametros de Red

\begin{tabular}{|c|c|c|c|c|c|}
\hline Potential & $\mathbf{a}[\AA]$ & $S i-O[\AA]$ & $S i-S i[\AA]$ & $\angle S i-O-S i$ & $\angle O-S i-O$ \\
\hline \multirow[t]{2}{*}{ SLC } & 11.85 & $1.587-1.604$ & $3.081-3.115$ & $147.961-157.845$ & $107.740-110.104$ \\
\hline & & 1.599 & 3.091 & 150.449 & 109.473 \\
\hline \multirow[t]{2}{*}{ SS96 } & 12.00 & $1.600-1.615$ & $3.112-3.159$ & $148.930-161.490$ & $108.656-110.485$ \\
\hline & & 1.611 & 3.131 & 153.07 & 109.5 \\
\hline \multirow[t]{2}{*}{ SS97 } & 12.03 & $1.620-1.632$ & $3.122-3.165$ & $145.981-153.361$ & $108.188-110.188$ \\
\hline & & 1.628 & 3.140 & 149.58 & 109.5 \\
\hline \multirow[t]{2}{*}{ Gale } & 11.88 & $1.584-1.609$ & $3.092-3.114$ & $148.482-158.854$ & $108.668-110.229$ \\
\hline & & 1.602 & 3.102 & 151.38 & 109.5 \\
\hline \multirow[t]{2}{*}{ SC1 } & 11.95 & $1.604-1.620$ & $3.144-3.109$ & $147.369-157.063$ & $107.662-110.268$ \\
\hline & & 1.615 & 3.118 & 150.015 & 109.473 \\
\hline \multirow[t]{2}{*}{$\mathrm{SC2}$} & 11.87 & $1.605-1.606$ & $3.062-3.123$ & $145.105-153.156$ & $108.295-109.724$ \\
\hline & & 1.605 & 3.093 & 149.200 & 109.470 \\
\hline \multirow[t]{2}{*}{ JC1 } & 11.84 & $1.589-1.604$ & $3.080-3.109$ & $147.419-156.086$ & $108.098-110.099$ \\
\hline & & 1.599 & 3.090 & 150.259 & 109.472 \\
\hline \multirow[t]{2}{*}{ JC2 } & 12.12 & $1.594-1.612$ & $3.137-3.176$ & $153.131-171.069$ & $104.487-116.796$ \\
\hline & & 1.607 & 3.167 & 161.291 & 109.233 \\
\hline \multirow[t]{2}{*}{ DSQFG } & 11.91 & 1.604 & $3.080-3.152$ & $147.412-158.539$ & $109.468-109.472$ \\
\hline & & 1.604 & 3.103 & 150.94 & 109.5 \\
\hline \multirow[t]{2}{*}{ JA } & 12.05 & $1.596-1.617$ & $3.182-3.136$ & $151.900-160.756$ & $106.097-111.760$ \\
\hline & & 1.611 & 3.148 & 155.78 & 109.3 \\
\hline \multirow[t]{2}{*}{ AHCM } & 11.67 & $1.538-1.561$ & $3.028-3.058$ & $152.871-167.078$ & $105.974-114.974$ \\
\hline & & 1.554 & 3.050 & 158.32 & 109.3 \\
\hline \multirow[t]{2}{*}{ BKS } & 12.08 & $1.590-1.617$ & $3.131-3.166$ & $152.050-167.485$ & $105.535-111.811$ \\
\hline & & 1.609 & 3.156 & 158.18 & 109.3 \\
\hline \multirow[t]{2}{*}{ Vessal } & 12.11 & $1.575-1.624$ & $3.141-3.200$ & $153.064-171.373$ & $104.178-117.218$ \\
\hline & & 1.610 & 3.172 & 161.140 & 109.197 \\
\hline \multirow[t]{2}{*}{ PMM06 } & 11.34 & $1.498-1.499$ & $2.919-2.993$ & $153.899-173.150$ & $103.061-118.545$ \\
\hline & & 1.499 & 2.959 & 162.960 & 109.189 \\
\hline \multirow[t]{2}{*}{ PMM08 } & 12.02 & $1.620-1.632$ & $3.109-3.129$ & $144.611-158.925$ & $109.064-110.370$ \\
\hline & & 1.628 & 3.137 & 149.554 & 109.467 \\
\hline \multirow[t]{2}{*}{ TTAM } & 12.358 & $1.624-1.654$ & $3.198-3.246$ & $151.646-168.837$ & $105.182-115.155$ \\
\hline & & 1.645 & 3.230 & 158.944 & 109.314 \\
\hline $\operatorname{Exp}^{12}$ & 11.8671 & 1.60 & & 153.3 & 109.4 \\
\hline
\end{tabular}


Tabla A.11: Si-LTA: Propiedades Mecánicas

\begin{tabular}{lcccc}
\hline Potential & Bulk Modulus (GPa) & \multicolumn{3}{c}{ Elastic Constants [GPa] } \\
& & $\mathbf{C}_{11}$ & $\mathbf{C}_{44}$ & $\mathbf{C}_{13}$ \\
\hline SLC & 74.45 & 102.49 & 25.04 & 60.44 \\
SS96 & 89.56 & 114.29 & 19.79 & 77.19 \\
SS97 & 59.38 & 78.81 & 17.57 & 49.66 \\
Gale & 94.93 & 124.24 & 24.73 & 80.29 \\
SC1 & 85.73 & 114.44 & 24.99 & 71.37 \\
SC2 & 54.77 & 77.95 & 25.75 & 43.17 \\
JC1 & 66.80 & 86.72 & 16.34 & 56.84 \\
JC2 & 80.33 & 100.84 & 15.94 & 70.07 \\
DSQFG & 0.00 & 7.58 & 0.00 & -3.78 \\
AHCM & 91.68 & 113.88 & 13.87 & 80.58 \\
JA & 15.75 & 23.11 & -39.00 & 12.06 \\
BKS & 90.71 & 117.51 & 22.82 & 77.31 \\
Vessal & 106.31 & 154.66 & 43.01 & 43.01 \\
PMM06 & 77.79 & 86.21 & 5.70 & 73.57 \\
PMM08 & 25.96 & 38.1097 & 17.3072 & 19.8885 \\
TTAM & 75.40 & 98.43 & 19.32 & 63.88 \\
\hline
\end{tabular}

Tabla A.12: Si-LTA: Propiedades Dieléctricas

\begin{tabular}{lcc}
\hline Potential & $\begin{array}{c}\text { Static Dielectric } \\
\text { Constant } \epsilon_{11}^{0}\end{array}$ & $\begin{array}{c}\text { High Frequency } \\
\text { Dielectric Constant } \epsilon_{11}^{\infty}\end{array}$ \\
\hline SLC & 2.62 & 1.53 \\
SS96 & 2.37 & 1.39 \\
SS97 & 2.51 & 1.41 \\
Gale & 2.65 & 1.49 \\
SC1 & 2.03 & 1.44 \\
SC2 & 3.47 & 2.28 \\
JC1 & 3.079 & 1.56 \\
JC2 & 1.95 & - \\
DSQFG & 1.00 & - \\
AHCM & 1.46 & - \\
JA & 1.41 & - \\
BKS & 1.43 & - \\
Vessal & 2.26 & - \\
PMM06 & 1.74 & - \\
PMM08 & 1.81 & - \\
TTAM & 1.48 & - \\
\hline
\end{tabular}




\section{A.5. Zeolita SSZ-73: Parámetros Calculados y Experi- mentales}

Tabla A.13: Zeolita SSZ-73: Parámetros de Red

\begin{tabular}{|c|c|c|c|c|c|c|}
\hline Potential & $\mathbf{a}[\AA]$ & c [̊̊] & $S i-O[\AA]$ & $S i-S i[\AA]$ & $\angle S i-O-S i$ & $\angle O-S i-O$ \\
\hline \multirow[t]{2}{*}{ SLC } & 14.03 & 10.21 & $1.595-1.600$ & $3.079-3.124$ & $149.70-155.333$ & $107.516-111.050$ \\
\hline & & & 1.599 & 3.089 & 149.988 & 109.476 \\
\hline \multirow[t]{2}{*}{ SS96 } & 14.23 & 10.34 & $1.606-1.613$ & $3.128-3.131$ & $149.725-153.589$ & $109.153-110.736$ \\
\hline & & & 1.611 & 3.131 & 152.650 & 109.466 \\
\hline \multirow[t]{2}{*}{ SS97 } & 14.42 & 10.43 & $1.618-1.627$ & $3.169-3.184$ & $150.823-156.975$ & $107.736-111.631$ \\
\hline & & & 1.624 & 3.167 & 154.4 & 109.5 \\
\hline \multirow[t]{2}{*}{ Gale } & 14.08 & 10.24 & $1.594-1.604$ & $3.084-3.133$ & $150.702-155.751$ & $108.226-110.585$ \\
\hline & & & 1.602 & 3.100 & 150.811 & 109.474 \\
\hline \multirow[t]{2}{*}{ SC1 } & 14.156 & 10.31 & $1.612-1.616$ & $3.107-3.115$ & $148.968-155.579$ & $107.274-111.190$ \\
\hline & & & 1.615 & 3.117 & 149.655 & 109.476 \\
\hline \multirow[t]{2}{*}{$\mathrm{SC2}$} & 14.068 & 10.300 & $1.605-1.606$ & $3.090-3.155$ & $148.486-158.463$ & $108.474-110.198$ \\
\hline & & & 1.605 & 3.104 & 150.580 & 109.470 \\
\hline \multirow[t]{2}{*}{ JC1 } & 14.0123 & 10.237 & $1.596-1.601$ & $3.075-3.131$ & $148.969-157.384$ & $108.159-110.761$ \\
\hline & & & 1.599 & 3.090 & 150.263 & 109.473 \\
\hline \multirow[t]{2}{*}{ JC2 } & 14.4679 & 10.3767 & $1.596-1.628$ & $3.168-3.171$ & $158.069-162.367$ & $104.344-116.918$ \\
\hline & & & 1.607 & 3.167 & 160.749 & 109.359 \\
\hline \multirow[t]{2}{*}{ DSQFG } & 14.17 & 10.26 & 1.604 & $3.116-3.139$ & $152.395-156.195$ & $109.468-109.475$ \\
\hline & & & 1.604 & 3.112 & 151.907 & 109.471 \\
\hline \multirow[t]{2}{*}{ JA } & 14.64 & 9.92 & $1.597-1.614$ & $3.074-1.614$ & $144.422-172.856$ & $106.334-111.541$ \\
\hline & & & 1.610 & 3.140 & 157.774 & 109.393 \\
\hline \multirow[t]{2}{*}{ AHCM } & 13.98 & 9.87 & $1.540-1.558$ & $3.043-3.059$ & $154.980-161.195$ & $108.464-111.024$ \\
\hline & & & 1.553 & 3.044 & 157.697 & 109.391 \\
\hline \multirow[t]{2}{*}{ BKS } & 14.43 & 10.28 & $1.594-1.616$ & $3.149-3.161$ & $156.154-161.993$ & $106.080-114.073$ \\
\hline & & & 1.608 & 3.152 & 157.454 & 109.396 \\
\hline \multirow[t]{2}{*}{ Vessal } & 14.47 & 10.36 & $1.583-1.630$ & $3.141-3.202$ & $158.366-165.984$ & $104.456-117.175$ \\
\hline & & & 1.610 & 3.172 & 160.453 & 109.318 \\
\hline \multirow[t]{2}{*}{ PMM06 } & 13.564 & 9.668 & $1.497-1.501$ & $2.956-2.982$ & $160.169-167.940$ & $103.197-119.055$ \\
\hline & & & 1.499 & 2.962 & 162.716 & 109.381 \\
\hline \multirow[t]{2}{*}{ PMM08 } & 14.195650 & 10.473640 & $1.623-1.631$ & $3.100-3.216$ & $145.303-151.461$ & $108.551-110.408$ \\
\hline & & & 1.627 & 3.145 & 150.752 & 109.471 \\
\hline \multirow[t]{2}{*}{ TTAM } & 14.762 & 10.513 & $1.628-1.655$ & $3.235-3.206$ & $151.155-162.559$ & $106.573-114.541$ \\
\hline & & & 1.644 & 3.225 & 157.967 & 109.383 \\
\hline $\operatorname{Exp}^{10}$ & 14.1039 & 10.1875 & 1.595 & & 150.1 & \\
\hline
\end{tabular}


Tabla A.14: Zeolita SSZ-73: Propiedades Mecánicas

\begin{tabular}{lcccccc}
\hline Potential & Bulk Modulus (GPa) $^{*}$ & \multicolumn{5}{c}{ Elastic Constants [GPa] } \\
& & $\mathbf{C}_{11}$ & $\mathbf{C}_{33}$ & $\mathbf{C}_{44}$ & $\mathbf{C}_{66}$ & $\mathbf{C}_{13}$ \\
\hline SLC & 69.21 & 78.83 & 145.26 & 16.10 & 33.40 & 66.61 \\
SS96 & 78.57 & 86.47 & 143.24 & 14.10 & 25.73 & 79.56 \\
SS97 & 86.65 & 94.93 & 140.92 & 14.89 & 25.05 & 84.43 \\
Gale & 85.16 & 94.14 & 167.23 & 15.88 & 33.46 & 86.98 \\
SC1 & 77.43 & 86.44 & 167.13 & 15.34 & 34.27 & 80.19 \\
SC2 & 50.93 & 59.39 & 151.54 & 25.51 & 33.81 & 60.70 \\
JC1 & 67.78 & 91.90 & 118.79 & 29.95 & 42.28 & 47.69 \\
JC2 & 61.25 & 67.61 & 122.59 & 3.53 & 20.94 & 64.24 \\
DSQFG & - & - & - & - & - & - \\
AHCM & 97.05 & 98.18 & 118.46 & 20.47 & 32.53 & 94.25 \\
JA & 31.65 & 92.16 & 34.80 & -2.34 & 24.13 & 45.41 \\
BKS & 89.17 & 95.05 & 128.01 & 11.93 & 34.85 & 84.39 \\
Vessal & 11.41 & 135.97 & 164.34 & 37.25 & 60.17 & 90.15 \\
PMM06 & 81.69 & 85.19 & 92.75 & 4.83 & 7.30 & 78.74 \\
PMM08 & 26.46 & 30.0193 & 124.4830 & 83.3917 & 25.9051 & 43.3602 \\
TTAM & 73.67 & 78.88 & 103.73 & 10.71 & 29.16 & 68.90 \\
\hline & & & & &
\end{tabular}

Tabla A.15: Zeolitea SSZ-73: Propiedades Dieléctricas

\begin{tabular}{lcc}
\hline Potential & $\begin{array}{c}\text { Static Dielectric } \\
\text { Constant } \epsilon_{11}^{0}-\epsilon_{33}^{0}\end{array}$ & $\begin{array}{c}\text { High Frequency } \\
\text { Dielectric Constant } \epsilon_{11}^{\infty}-\epsilon_{33}^{\infty}\end{array}$ \\
\hline SLC & $2.81-3.06$ & $1.26-1.27$ \\
SS96 & $2.54-2.72$ & $1.43-1.44$ \\
SS97 & $2.43-2.55$ & 1.17 \\
Gale & $2.87-3.15$ & $1.53-1.55$ \\
SC1 & $2.16-2.30$ & $1.49-1.51$ \\
SC2 & $2.28-2.37$ & $1.88-1.96$ \\
JC1 & $3.362-3.923$ & $1.613-1.640$ \\
JC2 & $1.43-1.44$ & - \\
DSQFG & $1.00-1.00$ & - \\
AHCM & $1.51-1.515$ & - \\
JA & $1.46-1.46$ & - \\
BKS & $1.47-1.48$ & - \\
Vessal & $2.42-2.43$ & - \\
PMM06 & $1.82-1.83$ & - \\
PMM08 & $1.89-2.09$ & - \\
TTAM & $1.53-1.54$ & - \\
\hline
\end{tabular}




\section{A.6. Si-CHA: Parámetros Calculados y Experimentales}

\begin{tabular}{|c|c|c|c|c|c|c|}
\hline Potential & $\mathbf{a}[\AA]$ & 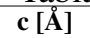 & $S i-O[\AA]$ & $S i-S i[\AA]$ & $\angle S i-O-S i$ & $\angle O-S i-O$ \\
\hline SLC & 13.55 & 14.58 & $1.598-1.606$ & $\begin{array}{c}3.052-3.090 \\
3.077\end{array}$ & $144.872-149.155$ & $108.091-111.170$ \\
\hline SS96 & 13.71 & 14.84 & $\begin{array}{c}1.610-1.617 \\
1.613\end{array}$ & $\begin{array}{c}3.094-3.129 \\
3.119\end{array}$ & $\begin{array}{c}147.426-151.822 \\
150.323\end{array}$ & $\begin{array}{c}108.642-110.183 \\
109.469\end{array}$ \\
\hline SS97 & 13.85 & 15.04 & $\begin{array}{c}1.622-1.629 \\
1.625\end{array}$ & $\begin{array}{c}3.131-3.165 \\
3.154\end{array}$ & $\begin{array}{c}149.194-153.679 \\
151.972\end{array}$ & $\begin{array}{c}107.579-111.286 \\
109.461\end{array}$ \\
\hline Gale & 13.60 & 14.61 & $\begin{array}{c}1.598-1.612 \\
1.605\end{array}$ & $\begin{array}{c}3.059-3.107 \\
3.089\end{array}$ & $\begin{array}{c}145.572-150.223 \\
148.570\end{array}$ & $\begin{array}{c}108.301-111.031 \\
109.475\end{array}$ \\
\hline SC1 & 13.67 & 14.77 & $\begin{array}{c}1.615-1.621 \\
1.619\end{array}$ & $\begin{array}{c}3.084-3.116 \\
3.109\end{array}$ & $\begin{array}{c}144.481-148.866 \\
147.456\end{array}$ & $\begin{array}{c}107.801-111.479 \\
109.473\end{array}$ \\
\hline $\mathrm{SC} 2$ & 13.62 & 14.71 & $\begin{array}{l}1.605 \\
1.605\end{array}$ & $\begin{array}{c}3.080-3.097 \\
3.091\end{array}$ & $\begin{array}{c}147.291-149.503 \\
148.663\end{array}$ & $\begin{array}{c}108.783-109.988 \\
109.470\end{array}$ \\
\hline JC2 & 13.87 & 15.06 & $\begin{array}{c}1.603-1.612 \\
1.608\end{array}$ & $\begin{array}{c}3.141-3.174 \\
3.159\end{array}$ & $\begin{array}{c}156.771-159.657 \\
158.413\end{array}$ & $\begin{array}{c}102.785-116.684 \\
109.341\end{array}$ \\
\hline JC1 & 13.54 & 14.55 & $\begin{array}{c}1.599-1.607 \\
1.603\end{array}$ & $\begin{array}{c}3.048-3.088 \\
3.073\end{array}$ & $\begin{array}{c}144.159-148.684 \\
147.039\end{array}$ & $\begin{array}{c}107.543-111.700 \\
109.474\end{array}$ \\
\hline DSQFG & 13.61 & 14.81 & $\begin{array}{l}1.604 \\
1.604\end{array}$ & $\begin{array}{c}3.082-3.108 \\
3.095\end{array}$ & $\begin{array}{c}147.785-151.271 \\
149.485\end{array}$ & $\begin{array}{c}109.465-109.477 \\
109.471\end{array}$ \\
\hline JA & 13.48 & 15.45 & $\begin{array}{c}1.602-1.620 \\
1.610\end{array}$ & $\begin{array}{c}3.033-3.193 \\
3.111\end{array}$ & $\begin{array}{c}139.704-170.537 \\
152.423\end{array}$ & $\begin{array}{c}104.368-113.519 \\
109.388\end{array}$ \\
\hline АНCM & 13.22 & 6.19 & $\begin{array}{c}1.544-1.776 \\
1.665\end{array}$ & $\begin{array}{c}2.998-3.174 \\
3.094\end{array}$ & $\begin{array}{c}118.800-165.644 \\
141.371\end{array}$ & $\begin{array}{c}74.324-180.000 \\
105.310\end{array}$ \\
\hline BKS & 13.85 & 14.82 & $\begin{array}{c}1.599-1.615 \\
1.609\end{array}$ & $\begin{array}{c}3.121-3.158 \\
3.140\end{array}$ & $\begin{array}{c}153.650-155.701 \\
154.852\end{array}$ & $\begin{array}{c}106.908-114.458 \\
109.391\end{array}$ \\
\hline Vessal & 13.85 & 15.09 & $\begin{array}{c}1.594-1.630 \\
1.611\end{array}$ & $\begin{array}{c}3.143-3.202 \\
3.163\end{array}$ & $\begin{array}{c}156.661-160.172 \\
157.979\end{array}$ & $\begin{array}{c}103.326-116.420 \\
109.303\end{array}$ \\
\hline РMM06 & 12.98 & 14.09 & $\begin{array}{c}1.498-1.499 \\
1.499\end{array}$ & $\begin{array}{l}2.939-2.958 \\
2.953\end{array}$ & $\begin{array}{c}157.3742-162.089 \\
160.440\end{array}$ & $\begin{array}{c}102.383-117.969 \\
109.361\end{array}$ \\
\hline PMМ08 & 13.83 & 14.81 & $\begin{array}{c}1.624-1.632 \\
1.627\end{array}$ & $\begin{array}{c}3.100-3.161 \\
3.137\end{array}$ & $\begin{array}{c}145.171-152.018 \\
149.231\end{array}$ & $\begin{array}{c}108.592-110.194 \\
109.472\end{array}$ \\
\hline TTAM & 14.184 & 15.128 & $\begin{array}{c}1.634-1.657 \\
1.645 \\
\end{array}$ & $\begin{array}{c}3.189-3.238 \\
3.214\end{array}$ & $\begin{array}{c}154.361-156.796 \\
155.444\end{array}$ & $\begin{array}{c}106.692-114.981 \\
109.374 \\
\end{array}$ \\
\hline $\operatorname{Exp}^{128}$ & 13.53 & 14.75 & 1.603 & & $148.4 / 148.0$ & 109.47 \\
\hline
\end{tabular}


Tabla A.17: Si-CHA: Propiedades Dieléctricas

\begin{tabular}{lcccccc}
\hline Potential & Bulk Modulus (GPa) $^{*}$ & \multicolumn{5}{c}{ Elastic Constants [GPa] } \\
& & $\mathbf{C}_{11}$ & $\mathbf{C}_{33}$ & $\mathbf{C}_{44}$ & $\mathbf{C}_{66}$ & $\mathbf{C}_{13}$ \\
\hline SLC & 71.0 & 122.10 & 72.21 & 27.87 & 25.67 & 6.12 \\
SS96 & 79.84 & 124.98 & 80.05 & 21.00 & 20.21 & 5.47 \\
SS97 & 84.107 & 124.30 & 84.70 & 20.31 & 19.50 & 5.28 \\
Gale & 83.98 & 144.97 & 83.98 & 27.32 & 25.37 & 6.22 \\
SC1 & 81.12 & 139.39 & 81.32 & 28.24 & 26.16 & 78.67 \\
SC2 & 74.76 & 119.87 & 76.86 & 29.19 & 21.63 & 67.75 \\
JC1 & 68.88 & 121.06 & 69.79 & 27.78 & 25.51 & 63.96 \\
JC2 & 81.72 & 102.22 & 88.94 & 16.32 & 15.15 & 4.13 \\
DSQFG & - & - & - & - & - & - \\
AHCM & 265.85 & 345.84 & 596.22 & 84.11 & 35.74 & -19.24 \\
JA & 17.11 & 25.82 & 61.20 & 7.75 & 2.59 & -0.14 \\
BKS & 59.56 & 126.19 & 65.23 & 24.93 & 20.86 & 3.43 \\
Vessal & 102.35 & 152.74 & 111.30 & 46.14 & 36.32 & 91.13 \\
PMM06 & 59.64 & 86.51 & 61.81 & 5.57 & 6.20 & 66.34 \\
PMM08 & 25.74 & 88.20 & 32.18 & 19.97 & 16.79 & 42.89 \\
TTAM & 46.76 & 103.70 & 51.68 & 20.96 & 17.72 & 60.64 \\
\hline
\end{tabular}

Tabla A.18: Si-CHA: Propiedades Dieléctricas

\begin{tabular}{lcc}
\hline Potential & $\begin{array}{c}\text { Static Dielectric } \\
\text { Constant } \epsilon_{11}^{0}\end{array}$ & $\begin{array}{c}\text { High Frequency } \\
\text { Dielectric Constant } \epsilon_{11}^{\infty}\end{array}$ \\
\hline SLC & 2.62 & 1.53 \\
SS96 & 2.37 & 1.39 \\
SS97 & 2.51 & 1.41 \\
Gale & 2.65 & 1.49 \\
SC1 & 2.18 & 1.48 \\
SC2 & 3.67 & 2.42 \\
JC1 & 2.93 & 1.59 \\
JC2 & 1.95 & - \\
DSQFG & 1.00 & - \\
AHCM & 1.46 & - \\
JA & 1.41 & - \\
BKS & 1.43 & - \\
Vessal & 2.37 & - \\
PMM06 & 1.81 & - \\
PMM08 & 1.91 & - \\
TTAM & 1.52 & - \\
\hline
\end{tabular}




\section{A.7. ITQ-1: Parámetros Calculados y Experimentales}

Tabla A.19: ITQ-1: Parámetros de Red

\begin{tabular}{|c|c|c|c|c|c|c|}
\hline Potential & $\mathbf{a}[\AA]$ & c [̊̊] & $S i-O[\AA \mathbf{\AA}]$ & $S i-S i[\AA]$ & $\angle S i-O-S i$ & $\angle O-S i-O$ \\
\hline SLC & 14.24 & 24.91 & $\begin{array}{c}1.586-1.615 \\
1.599\end{array}$ & $\begin{array}{c}3.079-3.099 \\
3.089\end{array}$ & $\begin{array}{c}147.339-152.485 \\
151.705\end{array}$ & $\begin{array}{c}104.151-111.399 \\
109.465\end{array}$ \\
\hline SS96 & 14.44 & 25.32 & $\begin{array}{c}1.607-1.622 \\
1.611\end{array}$ & $\begin{array}{c}3.090-3.186 \\
3.134\end{array}$ & $\begin{array}{c}147.859-165.336 \\
154.862\end{array}$ & $\begin{array}{c}107.748-112.104 \\
109.461\end{array}$ \\
\hline SS97 & 14.60 & 25.63 & $\begin{array}{c}1.618-1.630 \\
1.624\end{array}$ & $\begin{array}{c}3.134-3.219 \\
3.168\end{array}$ & $\begin{array}{c}148.031-159.370 \\
156.453\end{array}$ & $\begin{array}{c}106.564-113.495 \\
109.453\end{array}$ \\
\hline Gale & 14.30 & 25.03 & $\begin{array}{c}1.598-1.609 \\
1.601\end{array}$ & $\begin{array}{c}3.083-3.121 \\
3.104\end{array}$ & $\begin{array}{c}146.712-154.959 \\
153.306\end{array}$ & $\begin{array}{c}106.943-110.828 \\
109.464\end{array}$ \\
\hline SC1 & 14.39 & 25.17 & $\begin{array}{c}1.607-1.627 \\
1.614\end{array}$ & $\begin{array}{c}3.103-3.175 \\
3.121\end{array}$ & $\begin{array}{c}146.497-162.056 \\
152.140\end{array}$ & $\begin{array}{c}106.483-111.565 \\
109.465\end{array}$ \\
\hline $\mathrm{SC2}$ & 14.39 & 24.93 & $\begin{array}{c}1.598-1.610 \\
1.606\end{array}$ & $\begin{array}{c}3.064-3.216 \\
3.108\end{array}$ & $\begin{array}{c}151.511-180.000 \\
152.881\end{array}$ & $\begin{array}{c}108.207-110.777 \\
109.467\end{array}$ \\
\hline JC1 & 14.27 & 24.93 & $\begin{array}{c}1.578-1.608 \\
1.599\end{array}$ & $\begin{array}{c}3.089-3.156 \\
3.093\end{array}$ & $\begin{array}{c}149.178-180.000 \\
152.583\end{array}$ & $\begin{array}{c}105.016-110.864 \\
109.464\end{array}$ \\
\hline $\mathrm{JC2}$ & 14.57 & 25.65 & $\begin{array}{c}1.590- \\
1.606\end{array}$ & $\begin{array}{c}3.098-3.213 \\
3.167\end{array}$ & $\begin{array}{c}152.865-180.000 \\
162.067\end{array}$ & $\begin{array}{c}100.010-117.616 \\
109.322\end{array}$ \\
\hline DSQFG & 14.30 & 25.03 & $\begin{array}{c}1.604-1.605 \\
1.604\end{array}$ & $\begin{array}{c}2.845-3.208 \\
3.101\end{array}$ & $\begin{array}{l}179.936 \\
261\end{array}$ & $\begin{array}{c}109.349-109.735 \\
109.471\end{array}$ \\
\hline JA & 14.53 & 25.12 & $\begin{array}{c}1.589-1.628 \\
1.608\end{array}$ & $\begin{array}{c}3.045-3.194 \\
3.143\end{array}$ & $\begin{array}{c}141.001-179.878 \\
158.420\end{array}$ & $\begin{array}{c}102.784-114.433 \\
109.404\end{array}$ \\
\hline AHCM & 14.03 & 24.39 & $\begin{array}{c}1.540-1.559 \\
1.552\end{array}$ & $\begin{array}{c}2.979-3.099 \\
3.041\end{array}$ & $\begin{array}{c}145.587-180.000 \\
159.115\end{array}$ & $\begin{array}{c}102.605-115.053 \\
109.397\end{array}$ \\
\hline BKS & 14.51 & 25.39 & $\begin{array}{c}1.590-1.635 \\
1.607\end{array}$ & $\begin{array}{c}3.065-3.201 \\
3.151\end{array}$ & $\begin{array}{c}146.599-180.000 \\
159.155\end{array}$ & $\begin{array}{c}105.773-114.843 \\
109.404\end{array}$ \\
\hline Vessal & 14.56 & 25.59 & $\begin{array}{c}1.592-1.626 \\
1.609\end{array}$ & $\begin{array}{c}3.118-3.271 \\
3.172\end{array}$ & $\begin{array}{c}151.853-180.000 \\
162.003\end{array}$ & $\begin{array}{c}104.102-119.865 \\
109.324\end{array}$ \\
\hline PMM06 & 13.66 & 23.93 & $\begin{array}{c}1.491-1.509 \\
1.498\end{array}$ & $\begin{array}{c}2.912-2.980 \\
2.958\end{array}$ & $\begin{array}{c}153.706-180.000 \\
163.672\end{array}$ & $\begin{array}{c}102.351-113.940 \\
109.298\end{array}$ \\
\hline PMM08 & 14.55 & 25.37 & $\begin{array}{c}1.613-1.644 \\
1.627\end{array}$ & $\begin{array}{c}3.071-3.247 \\
3.152\end{array}$ & $\begin{array}{c}141.577-180.000 \\
153.568\end{array}$ & $\begin{array}{c}107.630-110.851 \\
109.467\end{array}$ \\
\hline TTAM & 17.63 & 29.31 & & & & \\
\hline $\operatorname{Exp}^{129}$ & 14.208 & 24.945 & $1.57-1.62$ & & $137-166$ & $107.0-111.6$ \\
\hline
\end{tabular}


Tabla A.20: ITQ-1: Propiedades Mecanicas

\begin{tabular}{|c|c|c|c|c|c|}
\hline \multirow[t]{2}{*}{ Potential } & \multirow[t]{2}{*}{ Bulk Modulus (GPa)* } & \multicolumn{4}{|c|}{ Elastic Constants [GPa] } \\
\hline & & $\mathbf{C}_{11}$ & $\mathbf{C}_{33}$ & $\mathbf{C}_{44}$ & $\mathbf{C}_{66}$ \\
\hline SLC & 73.20 & 143.11 & 90.20 & 13.08 & 37.68 \\
\hline SS96 & 86.47 & 140.24 & 103.23 & 12.78 & 26.23 \\
\hline SS97 & 89.13 & 138.79 & 107.32 & 12.71 & 25.11 \\
\hline Gale & 94.46 & 162.77 & 111.96 & 14.82 & 33.14 \\
\hline SC1 & 88.18 & 160.84 & 104.45 & 14.93 & 35.02 \\
\hline SC2 & 62.65 & 145.54 & 68.35 & 19.73 & 35.42 \\
\hline $\mathrm{JC2}$ & 76.05 & 113.81 & 91.96 & 11.07 & 19.42 \\
\hline JC1 & 66.98 & 116.84 & 78.09 & 13.23 & 21.78 \\
\hline DSQFG & 12.95 & 22.20 & -4.95 & 0.95 & -49.60 \\
\hline АНСM & 75.85 & 139.31 & 89.00 & 3.65 & 29.36 \\
\hline JA & 49.32 & 97.75 & 59.20 & -14.30 & 21.46 \\
\hline BKS & 81.92 & 140.40 & 103.58 & 11.64 & 31.84 \\
\hline Vessal & 100.39 & 175.26 & 147.93 & 24.61 & 47.67 \\
\hline PMM06 & 64.88 & 92.76 & 65.69 & -0.75 & 7.33 \\
\hline PMM08 & 60.71 & 116.91 & 72.02 & 7.56 & 26.39 \\
\hline TTAM & - & - & - & - & - \\
\hline
\end{tabular}

Tabla A.21: ITQ-1: Propiedades Dielectricas

\begin{tabular}{lcc}
\hline Potential & $\begin{array}{c}\text { Static Dielectric } \\
\text { Constant } \epsilon_{11}^{0}-\epsilon_{33}^{0}\end{array}$ & $\begin{array}{c}\text { High Frequency } \\
\text { Dielectric Constant } \epsilon_{11}^{\infty}-\epsilon_{33}^{\infty}\end{array}$ \\
\hline SLC & $3.24-2.84$ & $1.64-1.61$ \\
SS96 & $2.84-2.54$ & $1.45-1.43$ \\
SS97 & $1.39-1.38$ & - \\
Gale & $3.31-2.86$ & $1.56-1.54$ \\
SC1 & $2.37-2.16$ & $2.97-2.51$ \\
SC2 & $4.74-3.79$ & $1.65-1.62$ \\
JC1 & $4.07-3.31$ & - \\
JC2 & $2.17-2.08$ & - \\
DSQFG & $1.00-1.00$ & - \\
JA & $1.21-1.22$ & - \\
AHCM & $1.56-1.53$ & - \\
BKS & $1.51-1.49$ & - \\
Vessal & $2.54-2.45$ & - \\
PMM06 & $3.08-1.87$ & - \\
PMM08 & $2.05-1.90$ & - \\
TTAM & - & - \\
\hline
\end{tabular}


Parámetros Estructurales, Mecánicos y Dieléctricos de Estructuras Seleccionadas 


\section{Apéndice B}

\section{Lista de Artículos Publicados}

\section{B.1. Artículos que Hacen Parte de la Tesis}

1. Aldo F. Combariza and German Sastre. Structural and dynamical properties of silica polymorphs by interatomic potentials. In Preparation, October 2011.

2. Aldo F. Combariza and German Sastre. Influence of zeolite surface in the sorption of methane from molecular dynamics. Journal of Physical Chemistry C, 115(28):13751-13758, 2011.

3. Aldo F. Combariza, German Sastre, and Avelino Corma. Molecular dynamics simulations of the diffusion of small chain hydrocarbons in 8-ring zeolites. Journal of Physical Chemistry C, 115(4):875-884, 2011.

4. Aldo F. Combariza, German Sastre, and Avelino Corma. Propane/propylene diffusion in zeolites: Framework dynamics. Journal of Physical Chemistry C, 113(26):11246-11253, 2009.

5. Aldo F. Combariza, German Sastre, and Avelino Corma. Estimation of diffusional rates in tight fitting hydrocarbon-zeolite systems. DiffusionFundamentals.org, 11:26, 2009.

\section{B.2. Artículos que no Hacen Parte de la Tesis}

1. Diego A. Gomez, Aldo F. Combariza, and German Sastre. Confinement effects in $\mathrm{H} 2$ adsorbed in MOFs from molecular dynamics. Physical Chemistry Chemical Physics. Submitted, October 2011. 
2. Diego A. Gomez, Aldo F. Combariza, and German Sastre. Quantum-chemistry calculations of Hydrogen adsorption in MOF-5. Physical Chemistry Chemical Physics, 11(40):9250-9258, 2009.

\section{B.3. Artículos Publicados Durante M.Sc.}

1. Aldo F. Combariza, Ethan Sullivan, and Scott M. Auerbach. Simulating non-equilibrium dynamics of molecules confined in zeolite nanopores: Effects of implicit and explicit thermostats on microwave heated fluids. European Physical Journal: Special Topics, 141(1):93-99, 2007.

2. Aldo F. Combariza, Ethan Sullivan, Scott M. Auerbach, and Cristian Blanco. Simulating the relaxation dynamics of microwave-driven zeolites. Journal of Physical Chemistry B, 109(39):18439-18444, 2005. 


\section{Bibliografía}

[1] Agam, G.; Dagan, G.; Gilron, J.; Krakov, V.; Tsesin, N. Recovery of Olefins from Gaseous Mixtures, 2001, US Patent WO-2001-017664.

[2] Bryan, P. F. Sep. Pur. Rev. 2004, 33, 157-182.

[3] Eldrige, R. B. Ind. Eng. Chem. Res. 1993, 32, 2208-2212.

[4] Ren, T.; Patel, M.; Blok, K. Energy 2004, 31, 425-451.

[5] Azhin, M.; Kaghazchi, T.; Rahmani, M. Journal of Industrial and Engineering Chemistry 2008, 14, $622-638$.

[6] Jarvelin, H.; Fair, J. R. Ind. Eng. Chem. Res. 1993, 32, 2201-2207.

[7] Li, K.; Olson, D. H.; Seidel, J.; Emge, T. J.; Gong, H.; Zeng, H.; Li, J. Journal of the American Chemical Society 2009, 131, 10368-10369.

[8] Magalhaes Mendes, A. M.; Godinho De Faria Dos Santos, J. C.; Grilo Taveira, A. P. Device to Separate Olefins from Paraffins and to Purify Olefins and Use Thereof, 2009, US PAtent 20090270665.

[9] Ravanchi, M. T.; Kaghazchi, T.; Kargari, A.; Soleimani, M. Journal of the Taiwan Institute of Chemical Engineers 2009, 40, 511 - 517.

[10] Wragg, D. S.; Morris, R.; Burton, A. W.; Zones, S. I.; Ong, K.; Lee, G. Chem. Mater. 2007, 19, 3924-3932.

[11] Cantin, A.; Corma, A.; Leiva, S.; Rey, F.; Rius, J.; Valencia, S. J. Am. Chem. Soc. 2005, 127, 11560-11561.

[12] Corma, A.; Rey, F.; Rius, J.; Sabater, M. J.; Valencia, S. Nature 2004, 431, 287-290.

[13] Camblor, M. A.; Corma, A.; Lightfoot, P.; Villaescusa, L. A.; Wright, P. Angew. Chem. Int. Ed. 1997, 36, 2659-2661. 
[14] Eldridge, R. B. Ind. Eng. Chem. Res. 1993, 32, 2208-2212.

[15] Climent, M. J.; Corma, A.; Iborra, S. Chem. Rev. 2011, 111, 1072-1133.

[16] Corma, A. Nature 2009, 461, 182 - 183.

[17] Corma, A. J. Catal. 2003, 216, 298-312.

[18] Coronas, J. Chem. Eng. J. 2010, 156, 236 - 242.

[19] Baerlocher, C.; McCusker, L. B. Database of Zeolite Structures, http: //www.iza-structure.org/databases, 2011.

[20] Palomino, M.; Cantín, A.; Corma, A.; Leiva, S.; Rey, F.; Valencia, S. Chem. Comm. 2007, 1233-1235.

[21] Olson, D. H.; Camblor, M. A.; Villaescusa, L. A.; Kuehl, G. H. Microporous Mesoporous Mater. 2004, 67, 27-33.

[22] Barrett, P. A.; Boix, T.; Puche, M.; Olson, D. H.; Jordan, E.; Koller, H.; Camblor, M. A. Chemm. Commun. 2003, 2114-2115.

[23] Ruthven, D. M.; Reyes, S. Microporous Mesoporous Mater. 2007, 104, 59-66.

[24] Fick, A. E. Annalen der Physik und Chemie 1855, 94, 59.

[25] Fick, A. E. Philos. Mag. 1855, 10, 30.

[26] Graham, T. Quarterly Journal of Science, Literature and Art 1829, 27, 74.

[27] Graham, T. Philos. Mag. 1833, 2, 222.

[28] Dushman, S.; Langmuir, I. Phys. Rev. 1922, 20, 113.

[29] Langmuir, I. Phys. Rev. 1923, 22, 357-389.

[30] Arrhenius, S. Phys. Chemie 1889, 4, 226-248.

[31] Einstein, A. Annalen der Physik 1905, 17, 549.

[32] Einstein, A. Annalen der Physik 1906, 19, 371.

[33] Einstein, A. Z. für Elektrochemie 1907, 13, 98.

[34] Einstein, A. Z. für Elektrochemie 1908, 14, 235.

[35] Brown, R. New Phil. J. 1828, 5, 358-371. 
[36] Brown, R. Phil. Mag. 1828, 4, 161.

[37] Brown, R. Edin. J. Sci. 1829, 1, 314.

[38] Smoluchowsky, M. Annalen der Physik 1906, 21, 756.

[39] Smoluchowsky, M. Annalen der Physik 1906, 21, 756.

[40] Perrin, C. R. Acad. Sci. Paris 1908, 147, 475.

[41] Perrin, C. R. Acad. Sci. Paris 1908, 147, 530.

[42] Perrin, C. R. Ann. de Chim. et de Phys. 1909, 18, 1.

[43] Kelly, K. Science 1998, 279, 992-993.

[44] Koelling, D. DOE: Basis Energy Sciences Overview Brochures 2006, Core Research Activities, year.

[45] Miramontes, P. Un modelo de autómata celular para la evolución de los ácidos nucleicos, 1992.

[46] Hehre, W. J.; Radom, L.; v.R. Schleyer, P.; Pople, J. A. Ab Initio Molecular Orbital Theory; John Wiley and Sons: New York, 1986.

[47] Messiah, A. Quantum Mechanics; John Wiley \& Sons: New York, 1958; Vol. I, p 237.

[48] Arfken, G. Mathematical Methods for Physicists; Academic Press, Inc.: San Diego, 1985.

[49] Chandler, D. Introduction to Modern Statistical Mechanics; Oxford University Press: New York, 1987.

[50] Pechukas, P. Transition State Theory. In Dynamics of Molecular Collisions; Miller, W., Ed.; Plenum: New York, 1976; p 269.

[51] Statistical Mechanics; McQuarrie, D. A., Ed.; HarperCollins Publishers: New York, 1973.

[52] Modelling of Structure and Reactivity in Zeolites; Catlow, C. R. A., Ed.; Academic Press: London, 1992.

[53] Allen, M. F.; Tildesley, D. J. Computer Simulation of Liquids; Clarendon Press: Oxford, 1987. 
[54] Murch, G. E. In Diffusion in Crystalline Solids; Murch, G. E., Nowick, A. S., Eds.; Academic Press: Orlando, 1984; p 379.

[55] Post, M. F. Chapter 11 Diffusion in Zeolite Molecular Sieves. In Introduction to Zeolite Science and Practice; H. van Bekkum, E. F., Jansen, J., Eds.; Elsevier, 1991; Vol. 58, pp 391 - 443.

[56] Mehrer, H. diffusion-fundamentals.org 2009, 11, 1-32.

[57] Crank, J. The Mathematics of Diffusion; Clarendon Press, Oxford, 1956; p 347.

[58] Krishna, R. J. Phys. Chem. C 2009, 113, 19756-19781.

[59] Krishna. R.,; van Baten, J. M. Chem. Eng. Technol. 2007, 30, 1235.

[60] Kärger, J.; Pfeifer, H. Zeolites 1987, 7, 90-107, cited By (since 1996) 112.

[61] Krishna, R.; Baten, J. v. Microporous Mesoporous Mater. 2009, In Press, Corrected Proof, -.

[62] Metropolis, N.; Rosenbluth, A. W.; Rosenbluth, M. N.; Teller, A. H.; Teller, E. The Journal of Chemical Physics 1953, 21, 1087-1092.

[63] Alder, B. J.; Wainwright, T. E. The Journal of Chemical Physics 1959, 31, 459-466.

[64] Alder, B. J.; Wainwright, T. E. Proceedings of the International Symposium on Statistical Mechanical Theory of Transport Processes, 1958; p 97.

[65] Auerbach, S. M.; Jousse, F.; Vercauteren, D. P. Dynamics of Sorbed Molecules in Zeolites. Computer Modelling of Microporous and Mesoporous Materials, 2004.

[66] Smit, B. In Molecular simulations of the adsorption and diffusion of hydrocarbons in molecular sieves; Ruren Xu, J. C., Zi Gao, Yan, W., Eds.; Elsevier, 2007; Vol. Volume 170, Part 1, pp 121-129.

[67] Catlow, C. R. A.; Mackrod, W. C. Computer Simulation of Solids (Lecture Notes in Physics); Springer-Verlag, 1982; p 320 pages.

[68] Frenkel, D.; Smith, B. Understanding Molecular Simulation; Academic Press, 2002.

[69] Sanders, M. J.; Leslie, M.; Catlow, C. R. A. J. Chem. Soc., Chem. Commun. 1984, 1271-1273. 
[70] Demontis, P.; Suffritti, G. B.; Alberti, A.; Quartieri, S.; Fois, E. S.; Gamba, A. Zeolites 1987, 7, 522-527.

[71] Demontis, P.; Suffritti, G. B.; Alberti, A.; Quartieri, S.; Fois, E. S.; Gamba, A. J. Phys. Chem. B 1988, 92, 867-871.

[72] Jackson, R. A.; Catlow, C. R. A. Mol. Simul. 1988, 1, 207-224.

[73] Vessal, B.; Leslie, M.; Catlow, C. R. A. Mol. Sim. 1989, 3, 123-136.

[74] Duin, A. C. T. v.; Strachan, A.; Stewman, S.; Zhang, Q.; Xu, X.; Goddard, W. A. J. Phys. Chem. A 2003, 107, 3803-3811.

[75] Nomura, K.-i.; Kalia, R. K.; Nakano, A.; Vashishta, P. Computer Physics Communications 2008, 178, 73-87.

[76] Fogarty, J. C.; Aktulga, H. M.; Grama, A. Y.; van Duin, A. C. T.; Pandit, S. A. The Journal of Chemical Physics 2010, 132, 174704.

[77] Mahadevan, T. S.; Garofalini, S. H. The Journal of Physical Chemistry C 2008, 112, 1507-1515.

[78] Samatey, F. A.; Matsunami, H.; Imada, K.; Nagashima, S.; Shaikh, T. R.; Thomas, D. R.; Chen, J. Z.; DeRosier, D. J.; Kitao, A.; Namba, K. Nature 2004, 431, 1062-1068.

[79] Auerbach, S. M.; Ford, M. H.; Monson, P. Current Opinion in Colloid and Interface Science 2005, 10, 220 - 225.

[80] Rao, N. Z.; Gelb, L. D. The Journal of Physical Chemistry B 2004, 108, $12418-12428$.

[81] Jakobtorweihen, S.; Lowe, C. P.; Keil, F. J.; Smit, B. Journal of Chemical Physics 2007, 127, 024904.

[82] Zimmermann, N. E. R.; Jakobtorweihen, S.; Beerdsen, E.; Smit, B.; Keil, F. J. J. Phys. Chem. C 2007, 111, 17370-17381.

[83] Curtis, R. A.; Deem, M. W. J. Phys. Chem. B 2003, 107, 8612-8620.

[84] Sastre, G.; Catlow, C. R. A.; Chica, A.; Corma, A. J. Phys. Chem. B 2000, 104, 416-422.

[85] Corma, A.; Catlow, C. R. A.; Sastre, G. J. Phys. Chem. B 1998, 102, 70857090. 
[86] Sastre, G.; Cano, M. L.; Corma, A.; Garcia, H.; Nicolopoulos, S.; GonzalezCalbet, J. M.; ValletRegi, M. J. Phys. Chem. B 1997, 101, 10184-10190.

[87] Deem, M.; Newsam, J. M. J. Am. Chem. Soc. 1992, 114, 7189-7198.

[88] Liu, B.; Smit, B. Phys. Chem. Chem. Phys 2006, 8, 1852.

[89] GarciaPerez, E.; Dubbeldam, D.; Maesen, T. L. M.; Calero, S. J. Phys. Chem. B 2006, 110, 23968-23976.

[90] Smirnov, K. S.; Bougeard, D. Journal of Raman Spectroscopy 1993, 24, 255.

[91] March, N. H.; Totsi, M. P. Coulomb Liquids; Academic: New York, 1984.

[92] Revesz, A. G. Physical review. B, Condensed matter and materials physics 1971, 27, 1578-1581.

[93] Vessal, B. Journal of Non-Crystalline Solids 1994, 177, 103-124.

[94] Catlow, C. R. A.; Freeman, C. M.; Vessal, B.; Tomlinson, S. M.; Leslie, M. J. Chem. Soc. Faraday Trans. 1991, 87, 1947-1950.

[95] Schroder, K. P.; Sauer, J. J. Phys. Chem. 1996, 100, 11043-11049.

[96] Gale, J. D. J. Phys. Chem. B 1998, 102, 5423-5431.

[97] Ma, Y.; Garofalini, S. H. J. Chem. Phys. 2006, 124, 234102.

[98] Rappe, A. K.; Goddard, . I. I. I., W. A. The Journal of physical chemistry 1991, 95, 3358.

[99] Sefcik, J.; Demiralp, E.; Cagin, T.; Goddard, W. A. I. I. I. Journal of computational chemistry 2002, 23, 1507.

[100] Huang, M.-Z.; Lizhi, O.; Ching, W. Y. Physical Review B 1999, 59, 35403550 .

[101] Herzbach, D.; Binder, K.; Müser, M. H. J. Phys. Chem. 2005, 123, 124711.

[102] Zwijnenburg, M. A.; Cora, F.; Bell, R. G. J. Phys. Chem. B 2007, 111, 6156-6160.

[103] Muralidharan, K.; Cao, C.; Wan, Y.-X.; Runge, K.; Cheng, H.-P. Chem. Phys. Lett. 2007, 437, 92-98. 
[104] van Beest, B. W. H.; Kramer, G. J.; van Santen, R. A. Phys. Rev. Lett. 1990, 64, 1955-1958.

[105] Tsuneyuki, S.; Tsukada, M.; Aoki, H.; Matsui, Y. Phys. Rev. Lett. 1988, 61, 869-872.

[106] Kramer, G. J.; Farragher, N. P.; van Beest, B. W. H.; van Santen, R. A. Physical review. B, Condensed matter and materials physics 1991, 43, 50685080 .

[107] Demiralp, E.; Cagin, T.; Goddard, W. A. I. Phys. Rev. Lett. 1999, 82, 17081711.

[108] Tangney, P.; Scandolo, S. The Journal of chemical physics 2002, 117, 8898-8904.

[109] Wilson, M.; Madden, P. A.; Hemmati, M.; Angell, A. Phys. Rev. Lett. 1996, 77, 4023.

[110] Sastre, G.; Gale, J. D. Chem. Mater. 2003, 15, 1788.

[111] Flikkema, E.; Bromley, S. T. Chem. Phys. Lett. 2003, 378, 622-629.

[112] Auerbach, S. M.; Henson, N. J.; Cheetham, A. K.; Metiu, H. I. J. Phys. Chem. 1995, 99, 10600-10608.

[113] Jaramillo, E.; Auerbach, S. M. J. Phys. Chem. B 1999, 103, 9589-9594.

[114] Pedone, A.; Malavasi, G.; Menziani, M. C.; Cormack, A. N.; Segre, U. J. Phys. Chem. B 2006, 110, 11780-11795.

[115] Pedone, A.; Malvasi, G.; Menziani, M. C.; Segre, U.; Musso, F.; Corno, M.; Civarelli, B.; Ugliengo, P. Chem. Mater. 2008, 20, 2522.

[116] Sastre, G.; Corma, A. Journal of Physical Chemistry B 2006, 110, 1794917959.

[117] Sierka, M.; Sauer, J. Faraday Discussions 1997, 106, 41-62.

[118] Tilocca, A.; Leeuw, N. H. d.; Cormack, A. N. Phys. Rev. B 2006, 74, 104209.

[119] Tangney, P.; Scandolo, S. J. Chem. Phys. 2003, 119, 9673-9685.

[120] Olano, L. R.; Rick, S. W. Journal of Computational Chemistry 2005, 26, 699. 
[121] Ma, Y.; Garofalini, S. H. Mol. Simul. 2005, 31, 739.

[122] Ma, Y.; Garofalini, S. H. J. Chem. Phys. 2008, 128, 084505.

[123] Ma, Y.; Garofalini, S. H. Physical review. B, Condensed matter and materials physics 2006, 73, 174109.

[124] Rick, S. W.; Stuart, S. J.; Berne, B. J. Journal of Chemical Physics 1994, 101,6141 .

[125] Wright, A. F.; Lehmann, M. S. Journal of Solid State Chemistry 1981, 36, 371-380.

[126] Wyckoff, R. W. G. Crystal Structures; Interscience: New York, 1974.

[127] Smyth, J. R.; Smith, J. V.; Artioli, G.; Kvick, A. J. Phys. Chem. 1987, 91, 988-992.

[128] Diaz-Cabañas, M. J.; Barrett, P. A.; Camblor, M. A. Chem. Commun. 1998, 1882-1882.

[129] Camblor, M. A.; Corma, A.; Diaz-Cabañas, M. J.; Baerlocher, C. J. Phys. Chem. B 1998, 102, 44-51.

[130] Bachheimer, J. P. Journal of Physics Letters 1980, 41, 559-561.

[131] Dolino, G. Phase Transitions 1990, 21, 59-72.

[132] Gale, J. D.; Rohl, A. L. Mol. Simul. 2003, 29, 291.

[133] Gale, J. D. Journal of the Chemistry Society, Faraday Transactions 1997, 93, 629.

[134] Gale, J.; Sastre, G. Microporous Mesoporous Mater. 2001, 43, 27.

[135] Shanno, D. F. Math. Comp. 1970, 24, 647-656.

[136] Ewald, P. P. Ann. Phys. 1921, 64, 253.

[137] Toukmaji, A. Y.; Board, J. A. Computer Physics Communications 1996, $95,73-92$.

[138] Wolf, D. J. Chem. Phys. 1999, 110, 8254.

[139] Zicovich-Wilson, C. M.; Pascale, F.; Roetti, C.; Saunders, V. R.; Orlando, R.; Dovesi, R. Journal of Computational Chemistry 2004, 25, 18731881. 
[140] Hedin, N.; De Martin, G. J.; Roth, W. J.; Strohmaier, K. G.; Reyes, S. C. Microporous Mesoporous Mater. 2008, 109, 327-334.

[141] Hedin, N.; DeMartin, G. J.; Strohmaier, K. G.; Reyes, S. C. Microporous Mesoporous Mater. 2007, 98, 182-188.

[142] Eichler, U.; Kolmel, C. M.; Sauer, J. Journal of Computational Chemistry 1997, 18, 463-477.

[143] Sauer, J.; Schroeder, K. P.; Termath, V. Collection of Czechoslovak Chemm. Commun. 1998, 830, 1394-1408.

[144] Sastre, G.; Gale, J. Chem. Mater. 2005, 17, 730-740.

[145] Schrimpf, G.; Schlenkrich, M.; Brickman, J. J. Phys. Chem. 1992, 96, 7404-7410.

[146] Klein, H.; Fuess, H.; Schrimpf, G. J. Phys. Chem. 1996, 100, 1110111112.

[147] Keller, G. E.; Marcinkowsky, A. E.; Verma, S. K.; Williamson, K. D. Separation and Purification Technology; Marcel Dekker, 1992.

[148] Kärger, J.; Ruthven, D. M. Diffusion in Zeolites and Other Microporous Solids; John Wiley and Sons, 1992.

[149] Ruthven, D. M. Principles of Adsorption and Adsorption Processes; Wiley-Interscience, 1984.

[150] Krishna, R.; van Baten, J. M. Microporous Mesoporous Mater. 2011, 137, 83-91.

[151] ter Horst, J. H.; Bromley, S. T.; van Rosmalen, G. M.; Jansen, J. C. Microporous Mesoporous Mater. 2002, 53, 45-57.

[152] Demontis, P.; Suffritti, G. B. Chem. Rev. 1997, 97, 2845-2878.

[153] Demontis, P.; Suffritti, G. B. Microporous Mesoporous Mater. 2009, 125, $160-168$.

[154] Van Den Berg, B. S. R. N. M. T., A.W.C. Journal of Physical Chemistry B 2004, 108, 5088-5094, cited By (since 1996) 25.

[155] Van Den Berg, B. S. F. E. J. J., A.W.C. Journal of Chemical Physics 2004, 121, 10209-10216, cited By (since 1996) 9. 
[156] Smith, W.; Yong, C. W.; Rodger, P. M. Mol. Simul. 2002, 28, 385-471.

[157] Woodcock, D. A.; Ligthfoot, P.; Villaescusa, L. A.; Diaz-Cabañas, M. J.; Camblor, M. A.; Engberg, D. Chem. Mater. 1999, 11, 2508-2514.

[158] Oie, T.; Maggiora, T. M.; Christopherssen, R.; Duchamp, D. J. Int. J. Quantum Chem., Quantum Biol. Symp. 1981, 8, 1.

[159] Baerlocher, C.; McCusker, L.; Olson, D. H. Atlas of Zeolite Framework Types, 6th ed.; Elsevier: Amsterdam, 2007.

[160] Rohrbaugh, R. H.; Jurs, P. Anal. Chim. Acta 1987, 199, 99-109.

[161] Demontis, P.; Suffritti, G. B. J. Phys. Chem. B 1997, 101, 5789-5793.

[162] Fritzsche, S.; Haberlandt, R.; Hofmann, G.; arger, J.; Heinzinger, K.; Wolfsberg, M. Chem. Phys. Lett. 1997, 265, 253 - 258.

[163] Haberlandt, R. Thin Solid Films 1998, 330, 34-45.

[164] Astala, R.; Auerbach, S. M.; Monson, P. A. J. Phys. Chem. B 2004, 108, 9208-9215.

[165] Beerdsen, E.; Smit, B.; Dubbeldam, D. Phys. Rev. Lett. 2004, 93, 248301.

[166] Nagumo, R.; Takaba, H.; Nakao, S. J. Phys. Chem. C 2008, 112, 28052811.

[167] A. Corma,; J. Kärger,; C. Krause, Diffusionfundamentals.org 2005, 2, 87.1-87.2.

[168] Kärger, J. Microporous Mesoporous Mater. 2008, 109, 715-717.

[169] Shell, M. S.; Panagiotopoulos, A.; Pohorille, A. In Free Energy Calculations; Chipot, C., Pohorille, A., Eds.; Springer, 2007; Chapter Methods based on probability distributions and histograms, pp 77-116.

[170] Krishna, R.; van Baten, J. M. Chem. Eng. Technol. 2006, 29, 1429.

[171] Chmelik, C.; Heinke, L.; Valiullin, R.; Kärger, J. Chem. Ing. Tech. 2010, $82,779-804$.

[172] Chmelik, C.; Kärger, J. Chem. Soc. Rev. 2010, 39, 4864-4884.

[173] Chmelik, C.; Heinke, L.; Valiullin, R.; Kärger, J. Chem. Ing. Tech. 2010, $82,779-804$. 
[174] Ruthven, D. M.; Heinke, L.; Kärger, J. Microporous Mesoporous Mater. 2010, 132, 94-102.

[175] Zhang, L.; Chmelik, C.; van Laak, A. N. C.; Kärger, J.; de Jongh, P. E.; de Jong, K. P. Chem. Commun. 2009, 6424-6426.

[176] Fernandez, M.; Kärger, J.; Freude, D.; Pampel, A.; van Baten, J. M. v.; Krishna, R. Microporous Mesoporous Mater. 2007, 105, 124-131.

[177] Krutyeva, M.; Yang, X.; Vasenkov, S.; Kärger, J. J. Magn. Reson. 2007, $185,300-307$.

[178] Roque-Malherbe, R.; Polanco-Estrella, R.; Marquez-Linares, F. J. Phys. Chem. C 2010, 114, 17773-17787.

[179] Zimmermann, N. E. R.; Smit, B.; Keil, F. J. J. Phys. Chem. C 2010, 114, 300-310.

[180] Gren, W.; Parker, S. C.; Slater, B.; Lewis, D. W. J. Phys. Chem. C 2010, 114, 9739-9747.

[181] Karwacki, L. et al. Nature Mater. 2009, 8, 959 - 965.

[182] Thompho, S.; Chanajaree, R.; Remsungnen, T.; Hannongbua, S.; Bopp, P. A.; Fritzsche, S. J. Phys. Chem. A 2009, 113, 2004-2014, PMID: 19206222.

[183] Kortunov, P. V.; Heinke, L.; Arnold, M.; Nedellec, Y.; Jones, D. J.; Caro, J.; Kärger, J. J. Am. Chem. Soc. 2007, 129, 8041-8047, PMID: 17547400.

[184] Slater, B.; Titiloye, J. O.; Higgins, F. M.; Parker, S. C. Curr. Opin. Solid State Mater. Sci. 2001, 5, 417 - 424.

[185] Díaz, I.; Kokkoli, E.; Terasaki, O.; Tsapatsis, M. Chem. Mater. 2004, 16, 5226-5232.

[186] Ono, S. S.; Matsuoka, O.; Yamamoto, S. Microporous Mesoporous Mater. 2001, $48,103-110$.

[187] Wakihara, T.; Sasaki, Y.; Kato, H.; Ikuhara, Y.; Okubo, T. Phys. Chem. Chem. Phys. 2005, 7, 3416-3418.

[188] Abril, D. M.; Slater, B.; Blanco, C. Microporous Mesoporous Mater. 2009, $123,268-273$. 
[189] Slater, B.; Catlow, C. R. A.; Liu, Z.; Ohsuna, T.; Terasaki, O.; Camblor, M. A. Angew. Chem. Int. Ed. 2002, 41, 1235-1237.

[190] Baram P.S. and Parker S.C., Philos. Mag. B 1996, 73, 49-58.

[191] Combariza, A. F.; Sastre, G.; Corma, A. J. Phys. Chem. C 2009, 113, 11246-11253.

[192] Krishna, R.; van Baten, J. M. J. Phys. Chem. C 2010, 114, 18017-18021.

[193] Beerdsen, E.; Dubbeldam, D.; Smit, B. J. Phys. Chem. B 2006, 110, 22754-22772.

[194] Schüring, A.; Gulín-González, J.; Vasenkov, S.; Fritzsche, S. Microporous Mesoporous Mater. 2009, 125, 107 - 111, Diffusion in Micropores.

[195] Schüring, A. J. Phys. Chem. C 2007, 111, 11285-11290.

[196] Combariza, A. F.; Sastre, G.; Corma, A. J. Phys. Chem. C 2011, 115, 875884.

[197] Hibbe, F.; Chmelik, C.; Heinke, L.; Pramanik, S.; Li, J.; Ruthven, D. M.; Tzoulaki, D.; Kąrger, J. Journal of the American Chemical Society 2011, 133, 2804-2807.

[198] Heinke, L.; Kärger, J. Phys. Rev. Lett. 2011, 106, 074501.

[199] Choi, M.; Na, K.; Kim, J.; Sakamoto, Y.; Terasaki, O.; Ryoo, R. Nature 2009, 461, 246-249.

[200] Perez-Ramirez, J.; Christensen, C. H.; Egeblad, K.; Christensen, C. H.; Groen, J. C. Chem. Soc. Rev. 2008, 37, 2530-2542.

[201] Groen, J. C.; Zhu, W.; Brouwer, S.; Huynink, S. J.; Kapteijn, F.; Moulijn, J. A.; Pérez-Ramírez, J. Journal of the American Chemical Society 2007, 129, 355-360.

[202] Valiullin, R.; Kärger, J. Chemie Ingenieur Technik 2011, 83, 166-176.

[203] Levien, L.; Prewitt, C.; Weidner, D. J. American Mineralogist 1980, 65, 920-930.

[204] Bottom, V. Journal of Applied Physics 1975, 43, 1493-1495.

[205] Kammer, E. W.; Pardue, T. E.; Frissel, H. F. Journal of applied physics 1948, 19, 265-270. 
[206] Ohno, I.; Harada, K.; Yoshitomi, C. Phys. Chem. Min. 2006, 33, 1-9.

[207] Cook, R. K.; Weissler, P. G. Physical Review 1950, 80, 712-716.

[208] Liu, L.-g. Mechanics of Materials, 1993, 14, 283-290.

[209] Angel, R. J.; Mosenfelder, J. L.; Shaw, C. S. J. Physics of The Earth and Planetary Interiors, 2001, 124, 71-79.

[210] Mao, H.; Sundman, B.; Wang, Z.; Saxena, S. K. Journal of Alloys and Compounds 2001, 327, 253-262.

[211] Xu, Y.; Ching, W. Y. Physical review. B, Condensed matter and materials physics 1991, 44, 11048-11059. 